\title{
Cognitive-Inspired
}

\section{Visual Navigation System}

MICHAL AKIRA MUKAWA

School of Computer Science and Engineering

A thesis submitted to the Nanyang Technological University

in partial fulfilment of the requirement for the degree of

Doctor of Philosophy

2017 


\section{Acknowledgments}

Foremost, I would like to express sincere appreciation to my supervisor Dr. Lim Joo Hwee for his support, valuable and constructive suggestions during my research. His patience and willingness to give his time has been very much appreciated.

I thank Prof. Miao Chun Yan, my co-supervisor for giving thoughts, advices and ideas for improvement of my research. Also, I am sincerely grateful to her for supporting me in various university matters.

Also, I express my gratitude to my remaining TAC members: Prof. Tan Ah Hwee, Prof. Guan Cuntai, and Prof. Ong Yew Soon. Thank you for your time, effort, and support. I would also like to thank researchers from the Institute for Infocomm Research $\left(I^{2} R\right)$. In particular, Dr. Cheston Tan for his patient support, enthusiastic encouragement, and useful critique of my work; Dr. Xi Qianli, for all the support and useful insights related to design, conduct, and analysis of various user studies. Dr. Li Liyuan, for shaping the research progress.

I would also like to sincerely thank my fellow colleagues and other PhD students, past and present, in $\mathrm{I}^{2} \mathrm{R}$ and IPAL. Especially to Artsiom, Ana, Joaquim, Matt, Hanlin, Keng Teck, Gan Tian, Shue Ching, Olivier, Thibaut, Humayun, Stephane, Sepehr, and Hamdi. A huge thank you for the friendship and for making our lab an inspiring place.

Moreover, I would like to express my great gratitude to Aleksander Góra, Artur Matysik, and Marcin Dulęba. Thanks for your time, passion, and imagination to make Singapore a interesting place to be.

I wish to thank my wife Monika for her great support, patience, and understanding throughout these years. Last but not least, I would like to thank my parents for their encouragement and unconditional love. 


\section{Table of Contents}

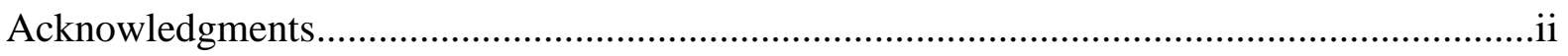

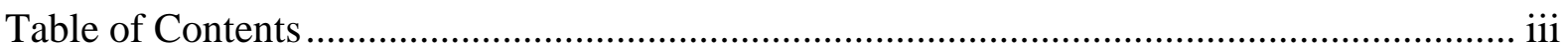

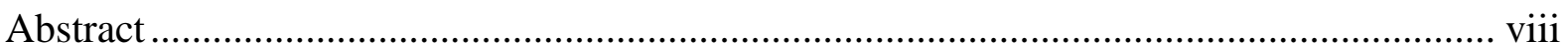

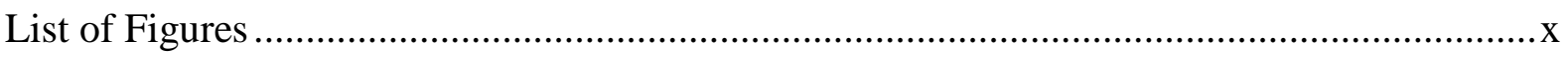

List of Tables .........................................................................................................

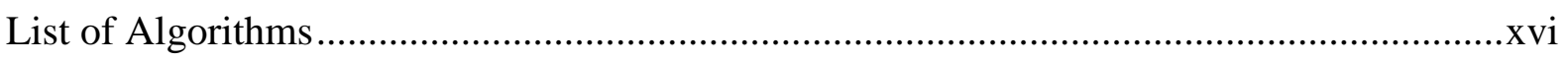

List of Publications ...............................................................................................

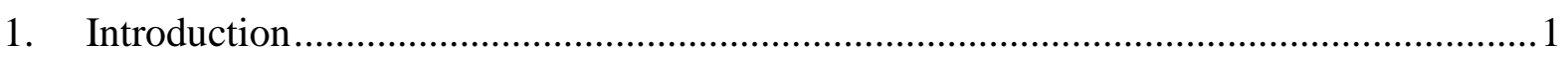

1.1. Indoor Navigation Challenge ....................................................................... 1

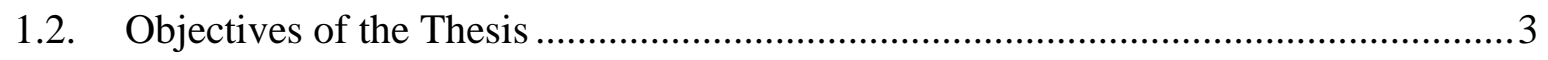

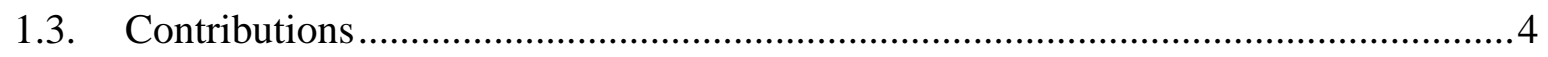

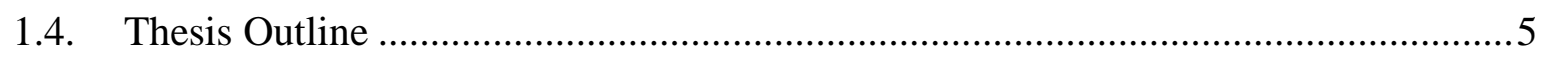

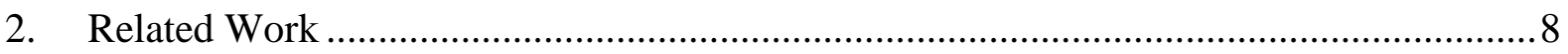

2.1. Human Memory Structure .............................................................................

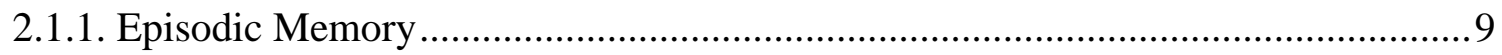

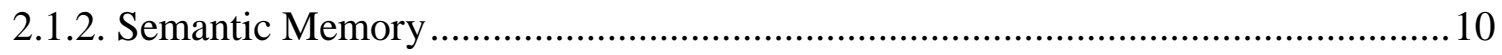

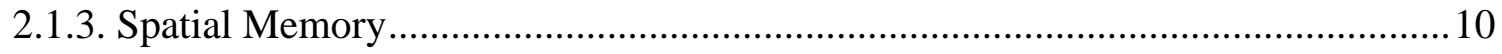

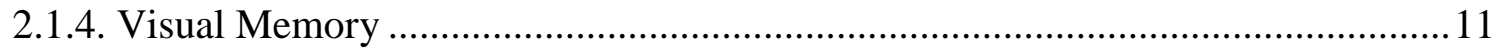

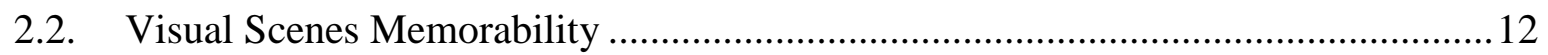

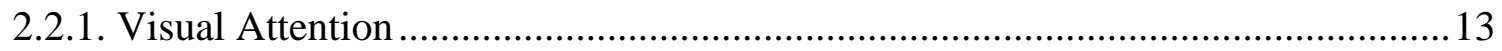




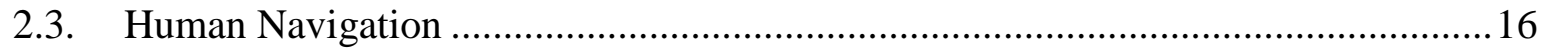

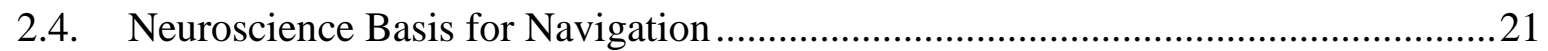

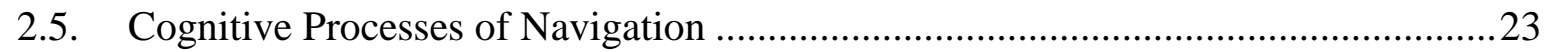

2.6. Indoor Localization and Navigation with Mobile Devices ..................................26

2.7. Different Interaction Modalities for Navigation Systems .....................................29

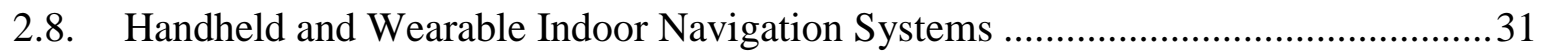

3. Scene Memorability and Visual Memory - Experiments ….............................................. 37

3.1. Navigation Assistance and Scene Memorability - Introduction and Methodology.. 37

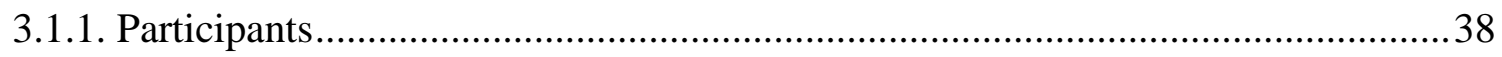

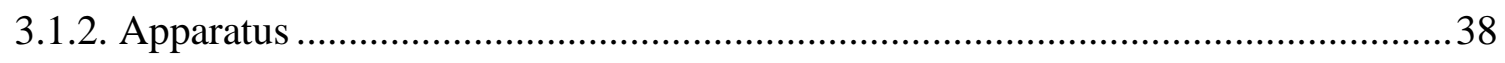

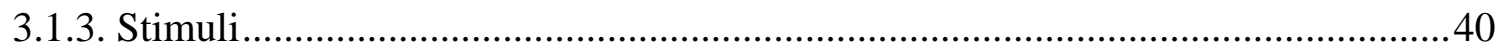

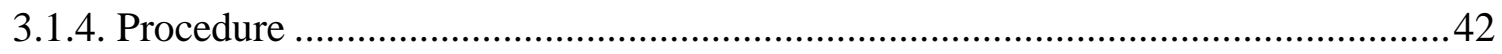

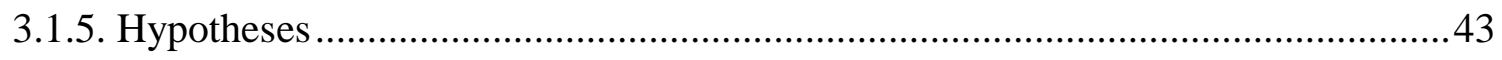

3.2. Navigation Assistance and Scene Memorability - Results ...................................46

3.2.1. Memorability of Scenes with Different Types of Navigation Assistance .............4 47

3.2.2. Memorability of Scenes with Different Navigation Information, and at Different

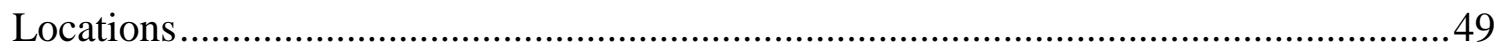

3.2.3. The Effect of Navigation Information on Scene Memorability ............................51

3.2.4. The Effect of Scene Location on Its Memorability ..........................................53

3.3. Navigation Assistance and Scene Memorability - Discussion ..............................56

3.3.1. Navigation Assistance Do Not Affect Scene Memorability .................................56 
3.4.1. Participants

3.4.2. Apparatus

3.4.3. Stimuli .66

3.4.4. Procedure .66

3.4.5. Hypotheses

3.5. Repetition Priming and Visual Memory - Results.

3.5.1. The effect of backtracking on visual memory

3.5.2. The effect of repetition priming on visual memory

3.6. Repetition Priming and Visual Memory - Discussion.

4. Do Navigation Assistance Interaction Modalities Affect Spatial Cognition?

4.1. Introduction and Methodology

4.1.1. Participants

4.1.2. Apparatus

4.1.3. Independent Variables .76

4.1.3.1. Navigation Assistance .76

4.1.3.2. Interaction Modality. .77 
4.1.5. Procedure .78

4.1.6. Hypotheses .80

4.2. Results .81

4.2.1. Navigation and Backtracking Performance 82

4.2.2. Correct Backtracking Decisions Performance. .86

4.2.3. Visual Memory Performance. .89

4.2.4. User Experience 91

4.3. Discussion 97

4.3.1. Visual Memory Is Not Affected by the Type of Navigation Assistance or Interaction Modality 97

4.3.2. Use the Voice+Display Modality for Best Navigation Performance, but Be Aware of Its Drawbacks .98

4.3.3. The Voice+Display Modality Is Perceived as Smarter and More Enjoyable to Use Than Human Guide 100

4.3.4. Limitations and Future Research Directions. 102

5. Towards a Novel Cognitive-Inspired Indoor Navigation System 105

5.1. Cognitive-Inspired Systems for Navigation with Visual Information Processing.. 105

5.1.1. DeSTIN 107

5.1.2. NOMAD 108

5.1.3. DUAL 108

5.1.4. ViSTARS 108 
5.1.7. ART

5.1.8. ACT-R

5.1.9. SAL

5.1.10. SOAR

5.2. Challenges Faced by Cognitive System for Navigation with Visual Input

5.3. Novel Cognitive-Inspired Indoor Navigation System 116

5.4. Cognitive-Inspired: Combine Egocentric and Allocentric Environment Representations

5.5. Scene Category Recognition using Pre-Trained CNN and SVM 120

5.6. Step and Turn Detection with Use of Accelerometer and Gyroscope

5.7. Create a Topological Map of an Environment from Google Glass Data

5.8. Guidance with Use of Google Glass and a Topological Map of an Environment.. 126

5.9. Conclusions 128

6. Conclusions and Future Perspectives. 130

References 134 


\begin{abstract}
This thesis summarizes current research on human navigation, with emphasis on human visual memory and spatial cognition. We study how different types of navigation assistance, different interaction modalities, and external stimuli (i.e., repetition priming) affect our navigation performance.
\end{abstract}

With the rapid growth of wearable computing devices, indoor navigation guidance will become popular in the near future like the GPS-based navigation tools for drivers today. However, how indoor navigation guidance affects human's memory of a novel environment has not been well studied. This thesis investigates route memory with three types of navigation assistance, i.e., 2D map, wearable navigation assistant, and human usher. The results show that the participants have similar patterns in remembering visual scenes, even using different types of assistance. These findings support previous work on scene memorability and provide the new insight that scene memorability is not affected by the type of navigation guidance. This may indicate that spatial working memory and visual memory are dissociated. We also show that scenes with navigation information are more memorable than scenes without such information. Finally, we provide some evidence that the location of a scene is linked to its memorability.

During wayfinding in a novel environment, we encounter many novel places. Some of those places are encoded by our spatial memory. But how does the human brain "decide" which locations are more important than others, and how do backtracking and repetition priming enhance memorization of these scenes? This thesis explores how backtracking improves encoding of encountered locations. We also investigate if repetition priming helps with further memory enhancement. The results show that backtracking alone significantly improves spatial memory of visited places. Surprisingly, repetition priming does not further 
enhance memorization of these places. This result may suggest that spatial reasoning causes significant cognitive load that thwarts further improvement of spatial memory of locations.

Next, as wearable devices, with a head-mounted display, become more popular, we noticed that there is a need to evaluate these devices as navigation systems and to investigate their effects on our spatial cognition. Our work investigates how Google Glass, with different interaction modalities (i.e., voice, display, and voice+display), affects spatial cognition during guided navigation. The results show that the voice+display modality can guide as effectively as Human Guide. However, this modality negatively affects backtracking. Also, the participants perceived higher system intelligence when using the voice+display modality. The results do not show any indication that the participants' visual memory is affected by any of the modalities.

Finally, we review cognitive systems that mimic human visual memory or navigation abilities. These systems play a vital role as a foundation for building cognitive-inspired, autonomous navigation systems. Next, we propose a novel, wearable, cognitive-inspired navigation system. The system is able to extract a topological representation of an explored environment and to provide efficient guidance through already mapped or pre-defined places. The topological structure is represented by visual scene categories (nodes of the topology) and their spatial relations (edges between nodes). Visual scene categories are detected by CNN (Convolutional Neural Network) with SVM (Support Vector Machine); while spatial relations are obtained from accelerometer and gyroscope data. To the best of our knowledge, it is the first design and demo of such a system. This system provides a strong foundation for efficient, real-life, navigation solutions that may be deployed in a number of scenarios (e.g., shopping malls, airports, offices, etc.). 


\section{List of Figures}

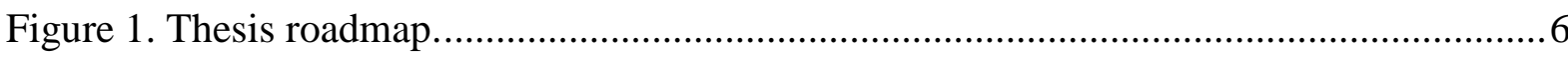

Figure 2. Pipeline of data flow in the human memory system. ............................................

Figure 3. Visual information flow in human memory system......................................... 13

Figure 4. Different sensory cues, computational mechanism, and spatial representations necessary for efficient human navigation. Adapted from (Wolbers \& Hegarty, 2010)...........20

Figure 5. Selected brain cells involved in navigation. a) Place cells - rat's path is shown as black line; red dots show firing field of observed place cell neurons. b) Head direction cells plot shows firing rate of an observed neuron for different orientations of the rat's head. c) Grid cells - red dots show firing fields of observed cells. Grid cells have multiple firing fields, which are distributed in a regular triangular grid - diagram (d). Adapted from Doeller, Barry, and Burgess (2012) .22

Figure 6. Landmark-based navigation system implemented on a mobile phone (Hile et al., 2008). .29

Figure 7. Different types of interaction modalities for navigation. a) and b) present a system which utilizes $3 \times 3$ tapping interface grid - contact speakers. Adapted from Ross and Blasch (2000). c) presents a tactile way-finding belt, which uses vibrating motors (Grierson, Zelek, \& Carnahan, 2009). d) presents a haptic glove, which utilize small vibrotactile motors adapted from Zelek (2005)..... 31

Figure 8. Google Indoor Maps navigation solution allows users to seamlessly switch between outdoor and indoor navigation.

Figure 9. Navigation system which utilize projector for guidance. The projector displays, in front of a user, navigation path. Adapted from Winkler, Broscheit, and Rukzio (2011)........34 
Figure 10. Navigation system that uses vision-based methods for localization - orange dots indicate recognized points. Guidance information is provided on the Google Glass display (green arrow). Adapted from U. Rehman and S. Cao (2015). .35

Figure 11. Two types of navigation assistance: (a) 2D map with marked destination e.g., the first navigation goal (star symbol); (b) wearable virtual navigator Mukawa et al. (2017)......39 Figure 12. Sample visited-scenes (top row) and unvisited-scenes (bottom row) images with annotations about their location and category of navigation information. Ambiguous info category image presents scene which contains number 10 - indicates floor number. This information can be wrongly interpreted by a participant as a location that he or she visited - if mostly semantic information (i.e., level 10) was remembered during navigation task. Adapted from Mukawa et al. (2017).

Figure 13. Scene memorability scores for different types of navigation assistance. MAP - 2D map; WVN - Wearable Virtual Navigator; HUG - Human Guide. Error bars show the standard error. Sample size $n=20$. Adapted from Mukawa et al. (2017). .48

Figure 14. Results of information categories at different locations. Error bars show standard error. Sample size $n=20$. Adapted from Mukawa et al. (2017). .52 Figure 15. Results of location categories for different information categories. Error bars show standard error. Sample size $n=20$. Adapted from Mukawa, Tan, Lim, Xu, and Li (2017)....54 Figure 16. Examples of visited scenes images with the memorability scores (mem. score). Memorability score indicates percent of the participants who correctly recognized particular scene. Sample size $n=20$. Adapted from Mukawa et al. (2017).

Figure 17. Average visual memory scores for the $1^{\text {st }}$ Group (at destination location and after backtracking) and for the $2^{\text {nd }}$ Group (only after backtracking) of the participants. Error bars show the standard error. Sample size $n=20$. Adapted from Mukawa et al. (2015). .68 
Figure 18. Correlations between scenes memorized by the different groups of participants. Adapted from Mukawa, Tan, Lim, Xu, and Li (2015). .70

Figure 19. Navigation assistance with use of Google Glass, a) a person wearing the Google Glass, b) the Google Glass, c) samples of navigation information displayed on the Google Glass display. 75

Figure 20. Mean navigation and backtracking times with the use of different types of assistance and different Google Glass modalities. Error bars show standard error. Sample size $\mathrm{n}=51$ (17 participants in each group - GG-Voice, GG-Display, GG-Voice+Display).

Figure 21. Average number of correct backtracking decisions with the use of different types of navigation assistance and different Google Glass modalities. Error bars show standard errors. Sample size $n=51$ (17 participants in each group - GG-Voice, GG-Display, GGVoice+Display)

Figure 22. Mean visual memory performance with the use of different types of navigation assistance and different Google Glass modalities. Error bars show standard error. Sample size $\mathrm{n}=51$ (17 participants in each group - GG-Voice, GG-Display, GG-Voice+Display) 90

Figure 23. Comparison of user experience measures for different modalities of providing navigation information with Google Glass. Error bars show standard errors. Sample size $n=$ 51 (17 participants in each group - GG-Voice, GG-Display, GG-Voice+Display) 91

Figure 24. Comparison of selected user experience measures for different types of navigation assistance and different Google Glass modalities. Error bars show standard error. Sample size $\mathrm{n}=51$ (17 participants in each group - GG-Voice, GG-Display, GG-Voice+Display)..........94 Figure 25. Most common structure of cognitive system. Based on Samsonovich (2010).... 107 Figure 26. Example of different cognitive architectures cooperation during navigation. ......115 Figure 27. Process of creating a topological structure of an environment with use of video and gyroscope data. First column shows how video frame category changes. Second column 
presents corresponding turn detections from gyroscope data. Finally, the last column, presents corresponding topology of the environment. 125

Figure 28. Navigation process with use of pre-created topological structure of an environment. Starting position is provided. Video and gyroscope data is used to define following positions within the topology. First column shows how video frame category changes. Second column presents corresponding turn detections from gyroscope data. Third column, presents corresponding topology of the environment together with current user's location. Finally, the last column, presents navigation commands provided to a user. 


\section{List of Tables}

Table 1 . Scene memory hypotheses with respect to our variables. (MAP - 2D map, WVN Wearable Virtual Navigator, HUG - Human guide). 46

Table 2. Mean memorability score (mean M), standard deviation, and number of images for each group of visited-scenes dataset. .50

Table 3. Mean false positive score (mean FP), standard deviation, and number of images for each group of unvisited-scenes dataset. .51

Table 4. Google Glass for Indoor Navigation - Experiment design .73

Table 5. Hypotheses for different interaction modalities (GG - Google Glass, HG - Human Guide). 81

Table 6. Navigation Time T-test Results Between Different Types of Navigation Assistance.

Table 7. Navigation Time ANOVA and T-test Results for Different Interaction Modalities of Google Glass .84

Table 8. Backtracking Time T-test Results Between Different Types of Navigation Assistance.

Table 9. Backtracking Time ANOVA Results for Different Interaction Modalities of Google

Glass .86

Table 10. Correct Backtracking Decisions T-test Results Between Different Types of Navigation Assistance

Table 11. Correct Backtracking Decisions ANOVA Results for Different Interaction Modalities of Google Glass. .88

Table 12. Memory Score T-test Results Between Different Types of Navigation Assistance. 
Table 13. Memory Score ANOVA Results for Different Interaction Modalities of Google Glass.

Table 14. User Experience Kruskal-Wallis and Mann-Whitney Tests Results for Different Interaction Modalities of Google Glass. 93

Table 15. User Experience Wilcoxon Tests Results Between Different Types of Navigation Assistance. .95

Table 16. User Experience Kruskal-Wallis and Mann-Whitney Tests Results for Significant Differences in Perceived Human Guidance. 97

Table 17. Memory types used in different cognitive systems. 106

Table 18. Possible navigation functions provided by various cognitive systems. 116 


\section{List of Algorithms}

Algorithm 1. Navigation Based on Pre-defined Environment Topology. 118

Algorithm 2. Create Topological Structure of an Environment from Google Glass Data. ... 119

Algorithm 3. Walking Detection. 122

Algorithm 4. Turn Detection... 123 


\section{List of Publications}

1. Mukawa, M., Tan, C., Lim, J. H, Xu, Q., Li, L. - "The Effect of Different Types of Navigation Assistance on Indoor Scene Memorability" - Behaviour and Information Technology, 2017

2. Mukawa, M., Xu, Q., Tan, C., Li, L., Lim, J. H. - "The Effect of Repetition Priming on Spatial Memory during Backtracking in a Novel Environment" - Cognitive Processing, 2015

3. Mukawa, M., Xu, Q., Tan, C., Li, L., Lim, J. H. - "The Influence of Different Types of Navigation Aid on Indoor Scene Memorability" - Proceedings of the $19^{\text {th }}$ Conference of the European Society for Cognitive Psychology, 2015

4. Mukawa, M., Lim, J. H. - “A Review of Cognitive Architectures for Visual Memory” Proceedings of the $5^{\text {th }}$ Annual International Conference on Biologically Inspired Cognitive Architectures, 2013

5. Xu, Q., Chia, S. C., Mandal, B., Li, L., Lim, J. H., Mukawa, M., Tan, C. - "SocioGlass: Social Interaction Assistance with Face Recognition on Google Glass" - Scientific Phone Apps and Mobile Devices, 2016

6. Li, L., Xu, Q., Chandrasekhar, V., Lim, J. H., Tan, C., Mukawa, M. - "A Wearable Virtual Usher for Vision-Based Cognitive Indoor Navigation" - IEEE Transactions on Cybernetics, 2016

7. Xu, Q., Mukawa, M., Li, L., Lim, J. H., Tan, C., Chia, S. C., Gan, T., Mandal, B. “Exploring Users' Attitudes towards Social Interaction Assistance on Google Glass” Proceedings of the $6^{\text {th }}$ Augmented Human International Conference, 2015

8. Ma, K. T., Dai, P., Lim, R., Li, L., Mukawa, M. - "Dataset for Unconstrained Visual Attention in First Person Videos" - $11^{\text {th }}$ Asia-Pacific Conference on Vision, 2015 
9. Xu, Q., Li, L., Lim, J. H., Tan, C., Mukawa, M., Wang, G. - “A Wearable Virtual Guide for Context-aware Cognitive Indoor Navigation" - Proceedings of the $16^{\text {th }}$ International Conference on Human-Computer Interaction with Mobile Devices and Services, 2014 


\section{Introduction}

\subsection{Indoor Navigation Challenge}

Navigation plays a vital role in our daily life. Most of us occasionally visit a new, unfamiliar environment, like an office building or airport. There are various possible reasons, such as a job interview, travel, shopping in a new mall, or visiting a famous skyscraper. Navigation in a novel indoor environment can be a demanding task and may require a considerable amount of attention.

Indoor guidance is especially challenging, when compare to outdoor guidance, as Global Positioning System (GPS) signal is barely reliable inside buildings to provide precise localization. It is caused by the fact that the GPS system achieves positioning function through satellites signals, which is easily obscured by the buildings and geographical environment (Misra \& Enge, 2006). Also, many navigation systems based on GPS signals are not precise enough for indoor application - while $10 \mathrm{~m}$ error may be acceptable outdoor, it would make 3 floors difference indoor. Therefore, for indoor navigation, we usually need to use some other kinds of localization methods. It can be signs located along a path, stationary maps that represent a building layout, or a human guide, just to mention a few. However, in recent years, wearable devices, like Google Glass, with displays appeared on the market. They show a high application potential for navigation assistance, both for outdoor and indoor environments.

Owing to the fast growth of mobile and wearable devices, the interest in navigation assistance has increased (May, Ross, Bayer, \& Tarkiainen, 2003). Indoor navigation solutions can support navigation in many scenarios, such as, helping tourists to find their points of interest, providing detour when the usual route is blocked, supporting rescue teams in a novel environment, or even providing guidance for people with special needs - disabled on a wheelchair (Millonig \& Schechtner, 2007). Moreover, indoor navigation has become 
important research area, as people use more location-based application, and indoor spaces are considered as most important for these applications (Costa et al., 2013). Still, the majority of portable indoor navigation systems provide allocentric information on a mobile screen (Arikawa, Konomi, \& Ohnishi, 2007; Serra, Carboni, \& Marotto, 2010). Compared with a traditional 2D map, or a digital map displayed on a smart phone screen, wearable devices equipped with a display provide allocentric or egocentric information on top of the user's field of view; therefore, it leads to less visual attention switch between the navigation information (i.e., 2D map, smart phone screen) and the environment.

Moller, Engelbrecht, Kuhnel, Wechsung, and Weiss (2009) pointed out that not only quality of service (i.e., the extent to which navigation information is reliable and delivered successfully), but users' quality of experience (i.e., how the navigation information is delivered and interpreted by the user) also plays a significant role in better usability and higher adoption rates. Human factors regarding outdoor navigation systems have been investigated (Pahlavan, Li, \& Mäkelä, 2002). However, there is a strong need to evaluate the effects of indoor navigation systems (Brown \& Pinchin, 2013). Usability and acceptance issues should also be addressed, so that users could perceive the benefits of such assistance.

Moreover, prior studies have not fully examined how different types of navigation assistance affect our memory during exploration of a novel indoor environment. Previous work on image memorability provides some interesting insights. However, these studies investigate which images people memorize when they are deliberately asked to do so. Typically, the participants view a long image sequence, consisting of a few thousand pictures. These images cover a wide range of categories, from objects, to faces, to landscapes. Moreover, the participants perceived stimuli on various kinds of displays, rather than in the real-world. Also, none of the studies on image memorability investigate how different experiment settings influence memorability. In general, findings provided by previous studies 
on image memorability cannot be used to forecast which scenes we memorize during exploration of a novel environment. This is partially because memorization of the scenes during navigation is a secondary task and often happens automatically without purposeful effort.

Therefore, first of our experiments examines if different types of navigation assistance (i.e., map, wearable navigation system, human guide) affect memorability of indoor visual scenes. Our second study, investigates if (and if yes, how) backtracking and repetition priming enhance our memory of visited places. We try to analyse if repetition priming can support our memory of visited places, when compared to backtracking alone. Our next experiment investigates if Google Glass (GG) is able to provide navigation assistance as efficiently as a person familiar with an environment - Human Guide (HG). Also, this study investigates effect of different GG interaction modalities (i.e., voice, display, voice+display) on participants' spatial abilities.

Finally, we review cognitive systems that mimic human visual memory or navigation abilities, as these systems play a vital role in building cognitive-inspired, autonomous navigation systems. Also, we propose a novel, wearable, cognitive-inspired navigation system, which is able to extract a topological representation of an explored environment and to provide efficient guidance through already mapped or pre-defined places.

\subsection{Objectives of the Thesis}

With a growing number of wearable devices (e.g., Google Glass), which can also be utilized to provide navigation, it is particularly interesting to investigate if (and how) these new systems impact our cognitive abilities. In particular, we are interested to investigate how do these devices influence our visual memory of a novel environment? Will these systems increase or decrease scene memorability during guided navigation? Will we remember the same or different scenes when using different forms of assistance? In particular, we 
investigate the influence of three types of navigation assistance (i.e., a map, a wearable navigation system, and a human guide) on indoor scene memorability. Furthermore, we are curious if people remember scenes that contain navigation information; also, we want to investigate if a scene location can predict how well the scene will be memorized. Moreover, it is worth exploring if cognitive load-caused by different types of navigation assistance affects scene memorability, i.e. is our visual memory influenced by the cognitive load? Also, we are interested to assess if backtracking or repetition priming enhance memorization of encountered visual scenes.

As wearable devices provide multiple ways of interaction (i.e., voice, display, or combination of both), it is of special interest to explore how these different interaction modalities affect spatial cognition during guided navigation. In particular, we investigate if Google Glass, with three different interaction modalities (voice, display, voice+display), affects user's spatial cognition during guided navigation, when compared to Human Guide. Also, we wanted to analyse, if Google Glass is able to provide navigation assistance as efficiently as a person. Moreover, as wearable devices are relatively novel technology, we are curious about subjective users' experience of using Google Glass as navigation assistance. For each experiment, we have reformulated our research objectives into hypotheses - to provide more details and references.

Finally, as part of our research, we want to propose a novel, wearable, cognitiveinspired navigation system. The system would be able to extract a topological representation of an explored environment and to provide efficient guidance through already mapped or predefined places.

\subsection{Contributions}

The main contributions of this thesis can be summarized in four parts, namely: 1) experimental study that investigate if different types of navigation assistance affect our 
memory during exploration of indoor environment, 2) experimental study that presents the effects of backtracking and repetition priming on our memory during guided navigation, 3) experimental study that evaluates different interaction modalities of Google Glass based indoor navigation system, compared them with Human Guide, and investigates their effects on our spatial cognition, 4) design and implementation of a novel cognitive-inspired, wearable, indoor navigation system.

\subsection{Thesis Outline}

This work is a multi-disciplinary intersection between cognitive science, human-computer interaction, and computer vision. It should be of interest to a wide range of readers interested in assistive indoor navigations, especially with the help of wearable devices. Figure 1 presents this thesis roadmap, which helps to navigate through the chapters. This thesis should be of interest to cognitive scientists, especially to those interested in our spatial cognition, in particular navigational abilities.

Although scene memorability is paramount in our navigation activities and relevant tasks, there is very limited literature that deals with the influence of different types of navigation assistance on indoor scene memorability. Therefore, in the Section 2.1 (Human Memory Structure), we briefly present different types of our memory and show how they cooperate. In the Section 2.2 (Visual Scenes Memorability), we review works on image memorability which show what kind of images - in general - we remember. Information provided in this section will show the importance of scene memorability in navigation task, and help to clarify the hypotheses in our experiments.

Next, in the Sections 2.3 (Human Navigation) 2.4 (Neuroscience Basis for Navigation), and 2.5 (Cognitive Processes of Navigation) we present findings on human navigation which address the human cognitive processes involved in navigation or compare navigation performance under different conditions. 


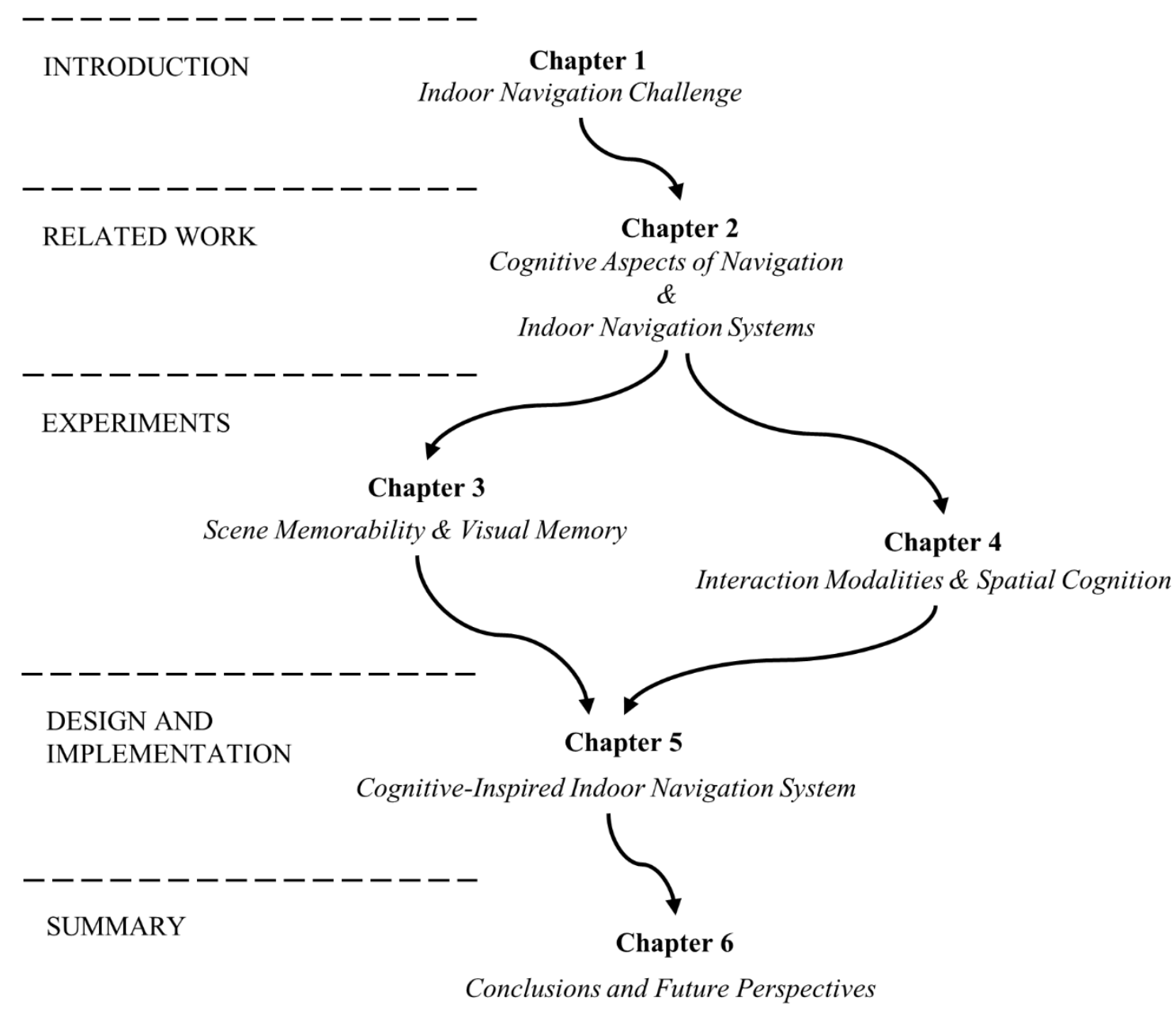

Figure 1. Thesis roadmap.

In the Section 2.6 (Indoor Localization and Navigation with Mobile Devices), we show current localization techniques, focusing on the challenges related to indoor navigation. The Sections 2.7 (Different Interaction Modalities for Navigation Systems) and 2.8 (Handheld and Wearable Indoor Navigation Systems), present different interaction modalities for navigation systems and current indoor navigation solutions.

In the following Chapter 3 (Scene Memorability and Visual Memory - Experiments), we analyse how different navigation systems (i.e., 2D map, wearable navigation assistant, and human usher) affect users' memory of visited places, and how this memory is affected by backtracking and repetition priming. 
In the Chapter 4 (Do Navigation Assistance Interaction Modalities Affect Spatial Cognition), we show how Google Glass, with different interaction modalities (i.e., voice, display, and voice+display), affects user's memory and spatial cognition during guided navigation. Also, the Glass-based system is compared against a Human Guide.

Finally, in the Chapter 5 (Towards a Novel Cognitive-Inspired Indoor Navigation System), we present a new vision- and sensors-based navigation system that mimics our cognitive abilities. Therefore, the system is able to create a topological structure of an environment, which may be later used to provide guidance to newcomers. 


\section{Related Work}

\subsection{Human Memory Structure}

Tulving and Craik (2000) provide a general definition of memory as the "neurocognitive capacity to encode, store, and retrieve information“, they also suggested that there may be many separate memory systems that fit this definition. Nowadays, memory systems are characterized by differences in timing, storage capacity, conscious access, active maintenance, and mechanisms of operation.

We, as humans, are only conscious of what is currently stored in our working memory. We are unconscious of things stored in the long-term memory (Sweller, Van Merrienboer, \& Paas, 1998). Through manipulation of data stored in our working memory we can perform cognitive tasks (A. Baddeley, 2000). All information currently stored in the working memory can be moved to long-term memory.

As memory and vision concern both the processes of the memory and nature of the stored representations, research in these domains seems to be especially interesting (Luck \& Hollingworth, 2008).

Figure 2 shows the data flow in the human brain. Sensory information is initially stored in sensory memory (John Robert Anderson, 2000), from which it can be encoded to working memory (A. D. Baddeley \& Hitch, 1974; Tulving \& Craik, 2000). At this stage, we become aware (i.e. conscious) of perceived stimuli. The data from working memory can be stored in long-term memory, from which working memory can later retrieve necessary information. Episodic and semantic memories are widely considered to be separate. Visual memory and spatial memory may overlap partially and support both episodic and semantic memory. 


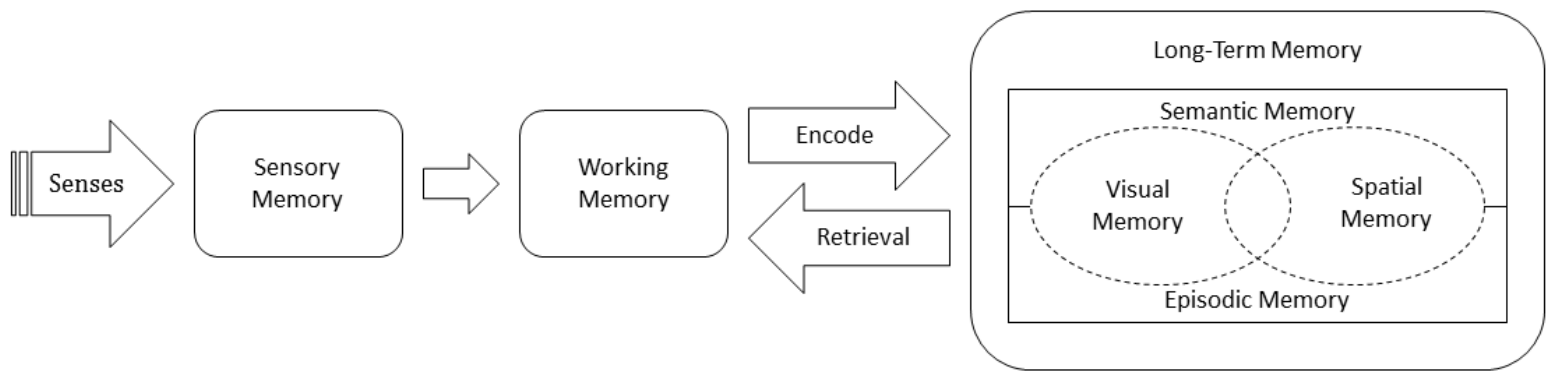

Figure 2. Pipeline of data flow in the human memory system.

Retrieval of memory can be based on sense of familiarity or the recollection (Yonelinas, 2002). The case of familiarity can be explained as example: "I saw this face before". And recollection can be explained in following way: "I remember that I met that person before". Familiarity, as well as, recollection is assumed to be continuous processes. One can say how certain he or she is in his or her familiarity or recollection measures (i.e. from weak to strong) (Eichenbaum, Yonelinas, \& Ranganath, 2007; Rugg \& Vilberg, 2012).

\subsubsection{Episodic Memory}

Episodic memory is a type of declarative, long-term memory that enables humans to remember their past experiences (Tulving, 1972, 2002). It includes contextualized information about specific events, such as the memory of your first trip abroad. It allows us to associate many different types of information (e.g. visual, auditory, tactile, etc.) into a single spatial-temporal context, to create our autobiographical timeline. The defining feature of episodic memory is that it allows us to travel mentally back in time and experience the event all over again.

Episodic memory allows us to consciously recall personal experiences and specific events that happened in the past. This includes recalling information like: when an event took place, where the event happened, what occurred during the event, or even the emotions associated with this event. Episodic memory is fully developed when we are around age four. 
Thanks to the episodic memory, we can recall layout of our home (floors plan), and even localize most furniture within this layout.

\subsubsection{Semantic Memory}

Semantic memory is also a type of declarative memory; it is what you "know". It consists of facts that are not linked to a specific experience, and are useful for reasoning about general properties of the world (Lahey et al., 2012). Semantic memory includes facts like the names of colours, the capitals of countries etc. The concept of semantic memory was introduced by Endel Tulving (1972).

Semantic memory is older than episodic memory in evolution. This is based on comparison of human beings with other mammals and birds who only have semantic memory but do not exhibit episodic memories. In addition, in human cognitive function development, semantic memory comes first. Children (2-3 years old) quickly gather knowledge about all sorts of things but do not have the capability to remember past events like adults do. Thanks to the semantic memory, we can anticipate seeing a lift lobby when entering, for a first time, a lobby of a new high office building. Even if we have never visited this particular building before, our abstract schema (stored in the semantic memory) of a typical high office building tell us what can we expect inside and which floor layouts are most probable.

\subsubsection{Spatial Memory}

Spatial memory contains information about the environment layout and spatial relations between entities. Environment and objects, stored in spatial memory, can be encoded by egocentric representation (related directly to the observer) and/or by allocentric representation (dependent on the relational position of objects in space) (Burgess, 2006; Klatzky, 1998). The allocentric representation is linked to a reference frame based on the external environment and independent of one's current location in it. For example, a 2D map of an environment is an allocentric representation. The egocentric representation is linked to a 
reference frame based on one's own location within the environment. For example, Google Glass and human guide are using egocentric representation while providing navigation, as when giving the direction as "please turn right" rather than "go north".

Moscovitch, Nadel, Winocur, Gilboa, and Rosenbaum (2006) presented a spatial memory framework that supports both episodic and semantic memories. In the former case, spatial memory provides detailed representations of the environment for autobiographical memories. In the latter case, general schematic representations of the environment are provided to support semantic memories. Hence, spatial memory helps us to localize and orientate ourselves during navigation by both positioning us in a specific mental map and guiding us with a general schematic map.

\subsubsection{Visual Memory}

Much has been described about the visual pathways in animals' eyes and brain. However, little has been said about high-level cognition involving visual memories, especially reconciling the visual information processing flow in the human brain. In unfettered environments, human vision is considered better than computer vision in many ways.

Visual working memory has a very limited capacity (only a few items), on the other hand visual long-term memory can retain thousands of items (Brady, Konkle, \& Alvarez, 2011). In general, visual memory allows us to recognize objects by matching them to similar objects that we have seen before (e.g. recognize that we are at a lift although it is the first time we see this particular lift). During navigation task, visual memory is responsible for recognizing familiar landmarks, and for encoding and storing new ones.

How is data stored in working memory? Brady et al. (2011) present one of the possibilities that initial encoding process is object-based (or location based), but that the "unit" of visual working memory is a hierarchically structured feature bundle: at the top level of individual "unit" is an integrated object representation; at the bottom level of an individual 
"unit" are low-level feature representations. In this same publication, authors provide evidence that individual items are not represented independently of other items shown on the same display; visual system efficiently extracts and encodes structure from the spatial and featural information across the visual scene.

Wood (2011) presents a system that uses three representations: spatio-temporal representation for object tracking, object property/kind representation for object recognition, and view-dependent snapshot representation for place recognition. Each of these systems may use its own buffer to keep information. Wood refers to this idea as "core knowledge architecture" of visual working memory.

\subsection{Visual Scenes Memorability}

Every day, we are exposed to huge amount of visual information. According to Koch et al. (2006) human retina may transfer data at roughly the rate of 10 million bits per second ( $\approx 10 \mathrm{Mbps}$ ). Our brain is not able to process that amount of visual information efficiently. Therefore, we use attention mechanism to focus on most important visual stimuli.

We present current findings on visual attention in the next subsection (see Visual Attention). While visual attention helps us to choose the most interesting (salient) or important (related to current task) visual entity, another brain mechanism evaluates its memorability.

Our brain is able to encode, store, and recall many visual memories. However, we do not remember all scenes or objects equally well. For example, we may remember what we dressed on our $18^{\text {th }}$ birthday party, but do not remember the face of a new office colleague, whom we met yesterday. 
Figure 3 presents generalized visual information flow in our brain. Scene information received through the eyes is temporary stored in sensory memory. Top-down and bottom-up attention decide which region in the scene should be processed next. At this stage, a representation of the perceived scene is created. When the conscious representation of a visual input is available in the working memory, it is evaluated in terms of memorability. Our brain needs to decide how useful currently perceived visual input is, and how important it may be for future tasks. Information stored temporarily in working memory may be transferred (encoded) to long-term memory. The higher the memorability prediction is the more likely this information will be encoded in long-term memory. Finally, working memory can hold information recalled from long-term memory. For example, recollection occurs when searching through visual long-term memory that visually matched the currently perceived scene, or when recalling episodic memories related to current visual information.

\subsubsection{Visual Attention}

Studies of attention and visual memory present peoples' limitations in encoding and maintaining visual information (Pashler, 1999). Attention affects which parts of visual scene will or will not be remembered; it impacts memorability. Therefore, in this section we present current findings on visual attention.

According to well-known psychologist and philosopher James (1890), attention can

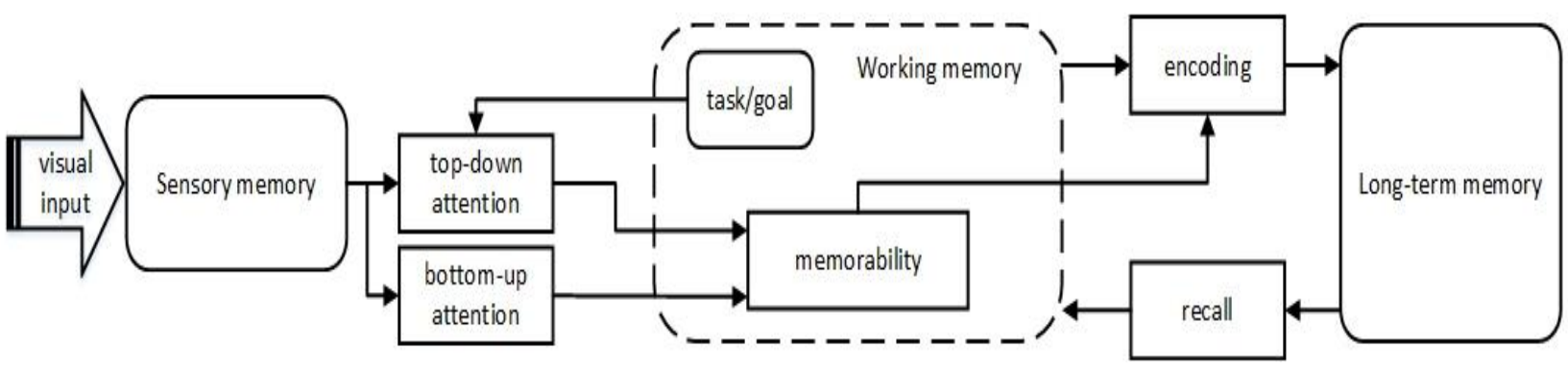

Figure 3. Visual information flow in human memory system. 
be defined as follow: "It is the taking possession of the mind, in clear and vivid form, of one out of what may seem several simultaneously possible objects or trains of thoughts. It implies withdrawal from some things in order to deal effectively with others" (p. 403 - 404). Therefore, attention can be considered as mechanisms by which relevant parts of sensory data are selected for further, more detailed processing; the rest are discarded. Attention controls the access to higher levels of processing, short-term memory, and consciousness. It is more than just noticing incoming stimuli. Attention involves many processes, like filtering our perceptions, balancing them (i.e. more important ones has priority before less important ones), or attaching emotional significance to these perceptions. In this study, we omit emotions' impact on attention because the mechanism and effect of emotion require significant separate effort and treatment.

Visual attention is one of our crucial perceptual mechanisms, as the human brain has limited resources and cannot process all visual input. Moreover, due to the brain's limited resources, human eyes are capable of perceiving high-resolution visual input only for about $2^{\circ}$ (i.e. visual input received by fovea) of our field of view (Fairchild, 2013). Peripheries are perceived in low resolution. Therefore, eye saccades (i.e. rapid movements of the eye from one fixation location to another) are also one of the attention's mechanisms. Attention helps us to decide where to move our eyes next.

There are two major forms of visual attention: bottom-up (passive) and top-down (active). Bottom-up attention visual attention is very rapid (approximately $100 \mathrm{~ms}$ ) (Itti, Koch, \& Niebur, 1998), primitive, and task-independent. It is an involuntary process directed by visual stimuli, like salient region (region which stands out among other regions) on the picture, or sudden motion pattern in the field of view. Top-down visual attention, is slower (approximately $200 \mathrm{~ms}$ ) (Connor, Egeth, \& Yantis, 2004), task-dependent, and under cognitive control. It is a multidimensional cognitive process that includes the ability to select 
and focus on what is important at given task. This complex process allows us to select what we should be attending to, in order to accomplish the task while ignoring task-irrelevant visual entities.

In terms of head, body, and eye movements, we can distinguish two types of attentions: overt and covert attention (Posner, 1980). Overt attention is related to head or eyes movements (e.g. when someone walks away from the painting in the museum). Covert attention happens without head or eyes movement. The attention is switched between different objects, or regions, while visual input remains the same.

Many models of visual attention have been developed (Borji \& Itti, 2013). By predicting which regions of visual input are more salient then others, computational model may focus on deeper analysis of those regions. Therefore, models of visual attention find application in many computer vision tasks, which requires such kind of information for efficient performance.

\subsubsection{Current Findings on Image Memorability}

Isola, Xiao, Torralba, and Oliva (2011) are one of the first researchers who attempt to connect image features with image memorability. By testing a few hundred subjects, they provided evidences that some images are more memorable than others, independent of the subjects' memories or other biases. By using only image features like GIST (Oliva \& Torralba, 2006), SIFT (Lowe, 2004), HOW2x2 (Dalal \& Triggs, 2005), and SSIM (Shechtman \& Irani, 2007), and by combining those features, they manage to predict (i.e. with Spearman's rank correlation $\rho=0.46$ ) image memorability.

In another study (Isola, Parikh, Torralba, \& Oliva, 2011) the authors tried to understand better intrinsic image memorability. They found that images which contain people with visible faces are more memorable, while images of wide vistas or calm scenes are less memorable. They provided surprising conclusion, that unusual or aesthetically pleasing 
scenes are not always highly memorable. They present general attributes, which can be used for picture description. Those attributes cover five categories: Spatial layout, Aesthetics, Emotions, Dynamics, and Location (each category has many multiply possible answers). There is additional attribute, which has only one answer, "Contains a person?". For those images, which contain a person, the additional set of attributes is available: visibility, demographics, appearance, clothing, accessories, etc.

Khosla, Xiao, Torralba, and Oliva (2012) proposed a probabilistic framework, that models which image regions are less memorable, and how weakly memorable they are. They used data-driven approach, which combines local and global images features. They extract information about gradient, colour, and texture from the image. Next feature memorability map is created for each of them. Those maps are pooled together to create overall memorability map. To verify that some image regions are more memorable, other studies Khosla, Xiao, Isola, Torralba, and Oliva (2012) modified image memorability, by changing some of its regions. They did it in two stages. In the first stage, they identified memorability of different image regions. In the second stage those regions were changed, to observe lower or higher (depends which regions have been changed) memorability of a new image.

Mancas and Le Meur (2013) inspired by the work described above, added saliency map information to check if that will improve memorability predictions. They combined image features information with subjects' eyes movements. The performance achieved (i.e. Spearman's rank correlation $\rho=0.48$ ) is slightly better than that (i.e. Spearman's rank correlation $\rho=0.46$ ) presented in the original paper by Isola, Parikh, et al. (2011).

\subsection{Human Navigation}

When we perform navigation tasks such as driving to work, we often do it unconsciously. We just "know" where we want to go and "how" to get there. In novel environments, we are able to learn fast and assimilate the general and detailed topology of a room, building, and the 
broader environment. Navigation is a capability that comes naturally for most of us, although it can degenerate during old age. However, this ability represents one of the most fundamental and complex humans' cognitive function. Indeed, navigation encompasses a family of inter-related tasks, and involves many brain regions. On everyday basis, we are unaware of this complex ability. We take our navigation ability for granted, assuming that we will always be able to use it efficiently. However, even small brain lesions may disrupt some of our navigation functions. This may lead to many daily problems, like finding way back home after an evening walk. This chapter presents what human navigation is and what cognitive neuroscience and psychology know about this process.

Navigation in general is defined as the process of controlling and monitoring the movement from one place to another (Torres-Méndez \& Cervantes-Jacobo, 2012). In this thesis we adopt more specific definition of human navigation proposed by Montello (2005): navigation consists of two components, locomotion and wayfinding. Locomotion refers to short-term navigation behaviour (body movement) in response to current position and local surrounding, whereas wayfinding refers to planning and decision making, which is based on some representation of the environment, and aims at reaching desired destination. From the perspective of time, it is a more long-term behaviour than locomotion. For efficient locomotion, a person needs to avoid obstacles, steers the body movements away from approaching objects. Wayfinding requires reasoning, planning, decision making, and some representation of the environment. Locomotion requires coordination of one's sensory and motor systems to move successfully in the intended direction without injuring oneself or moving into obstacles. During wayfinding, a person needs to constantly locate his or her position, checks if he or she is on the right path and direction by identifying specific landmarks. As locomotion depends mostly on coordination between sensory information and 
body movements (i.e. motorsensory functions and not so much on higher cognitive functions), in this thesis we focus on wayfinding aspect of human navigation.

Allen (1999) defines three types of wayfinding. He called the first one "oriented search", in this task a person starts navigation from a known location, and searches for a specific destination. The second type is called "route following". It can be well described with example of following painted lines on a supermarket floor that guides the person to the bakery section of the supermarket. The third category presented by Allen is "piloting from landmark to landmark". There might be a list of directions, which we receive from someone, when asking for direction to a specific place: e.g. go straight towards the city hall, turn left after the tallest building etc. In this type of navigation one landmark leads to another one. Wiener, Büchner, and Hölscher (2009) proposed extended wayfinding taxonomy. Wayfinding types were divided into different categories, based on the information available to the person, and the purpose of the navigation. The main distinction is between aided and unaided wayfinding. During aided wayfinding, the person follows a map, signs, GPS, or voice commands to reach his or her destination. Unaided wayfinding can be divided into undirected and directed modes. During undirected wayfinding there is no specific destination, while during directed wayfinding a destination is specified. Wiener et al. (2009) specified three deeper levels of directed wayfinding in his proposed taxonomy, but we will omit them, as they are out of the scope of this thesis.

Human navigation is regarded as a family of interrelated subtasks. Wolbers and Hegarty (2010) gathered and presented various sensory cues, computational mechanism, and spatial representations, which are necessary for efficient human navigation - there are presented in Figure 4. 
The list in Figure 4 presents the complexity of human navigation task. In this thesis, we focus on these cues, computations, and representations, which are crucial for wayfinding, and which can be obtained from visual input.

To perform computations required for human navigation system, some basic information is needed. The most crucial ones are: current location, heading direction, velocity, and some representation of the environment (e.g. spatial map). In theory, not all of these are required as raw inputs to a navigational system, because some can be computed from others. For example, if the location at any point in time is known, then heading direction and speed can be computed as the temporal derivative of location. Conversely, the current location can be identified by using the sensory cues of self-motion to update the distances and directions travelled from an arbitrary starting location. This process is known as "path integration” (McNaughton, Battaglia, Jensen, Moser, \& Moser, 2006). Similarly, a map can be constructed from scratch from the other raw ingredients, like an adventurer mapping unexplored territory.

The different raw ingredients may be redundant in theory, but in practice, there are trade-offs. Estimating current position by triangulating from known landmarks is highly dependent on map accuracy, while path integration is highly dependent on accurate perception of self-movement. In the relatively featureless desert environment, these trade-offs favour the use of path integration by desert ants (Müller \& Wehner, 1988). 

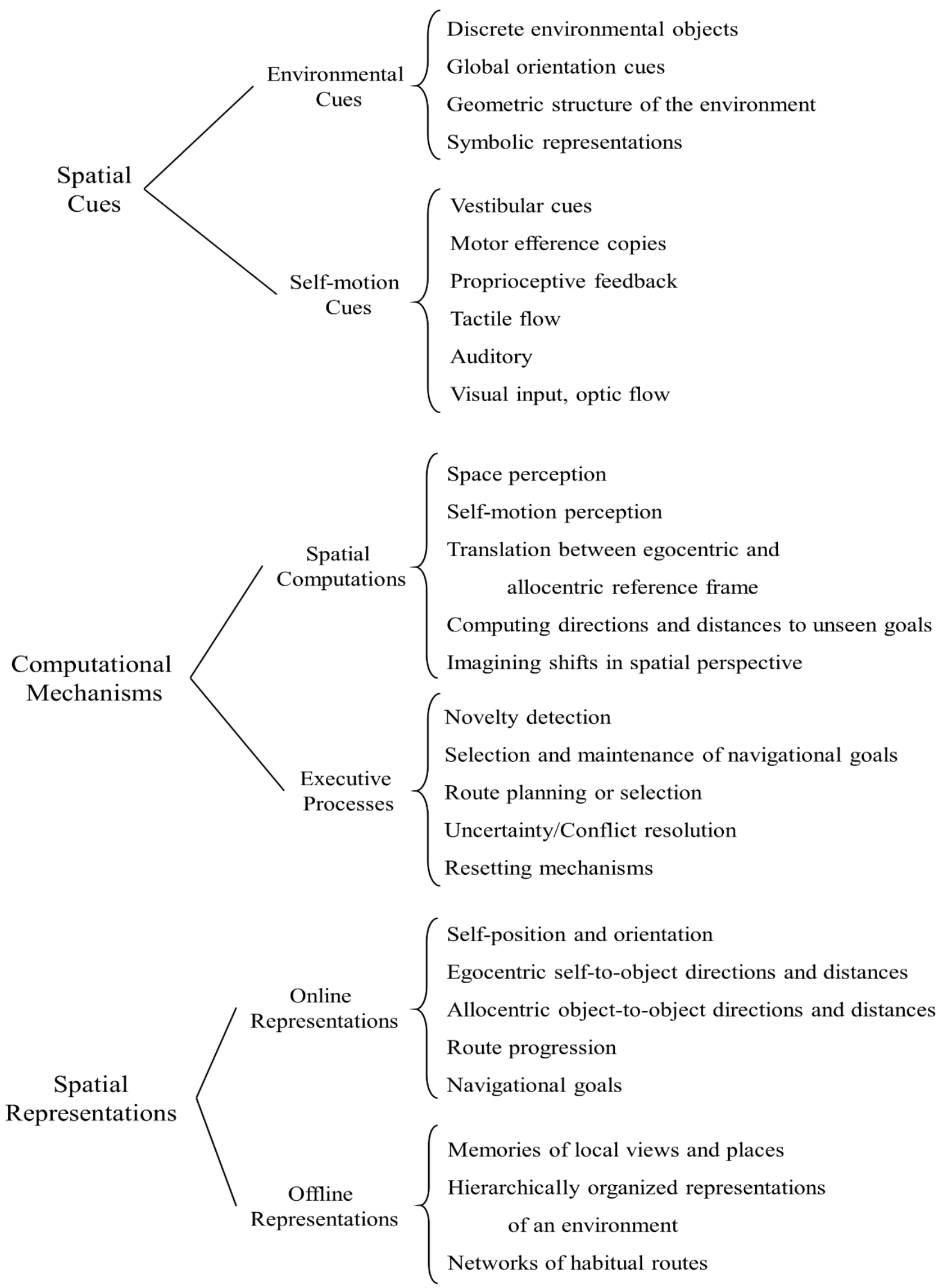

Figure 4. Different sensory cues, computational mechanism, and spatial representations necessary for efficient human navigation. Adapted from (Wolbers \& Hegarty, 2010). 


\subsection{Neuroscience Basis for Navigation}

The neural correlates of many of raw ingredients, mentioned in the previous section, have been found in animals and humans (i.e. biological systems capable of navigation). Current location is represented by "place cells" (O'Keefe \& Dostrovsky, 1971) with support of "grid cells" (Hafting, Fyhn, Molden, Moser, \& Moser, 2005). Heading is represented by "head direction cells" (Taube, 1995). These cells are illustrated in Figure 5 and will be described next.

O'Keefe and Dostrovsky (1971) observed that individual neurons fired when a rat was in a specific location in its environment. They called that kind of neurons "place cells". Their firing place (place field) is related to available visual landmarks: when the most silent landmark position is changed, the location where a particular place cell fires also changes. However, place cells keep their activities even when visual information is unavailable. Therefore, place cells can retain representation of different locations within the environment. This may be considered as cognitive map of nearest surrounding. Interesting finding was presented by Pfeiffer and Foster (2013) as they show that "place cells" play out the sequence, just before a rat starts the movement, which can be used to predict rat's future behaviour.

Head direction (HD) cell fires when the head is pointed in the "preferred" direction of that cell. The average range over which an HD cells would fire is approximately $84^{\circ}$ (Taube, 1995). Taube et al. also found that preferred firing directions of HD cells are anchored on the most silent landmark in the environment. When the position of the landmark is shifted, the same HD cell would now be firing with the new preferred direction (shifted accordingly to landmark shift). HD cells keep firing even when visual landmarks are unavailable, or when visual stimuli are absent. HD cells are also sensitive to the shape of the environment. When rats were moved from the cylindrical shape cage to rectangular one, their preferred firing direction changed significantly (at least by $78^{\circ}$ ). According to Stackman and Taube (1997), 


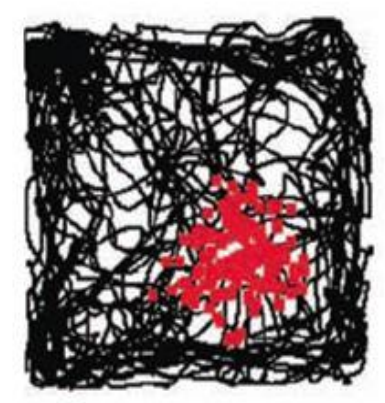

a) Place cells

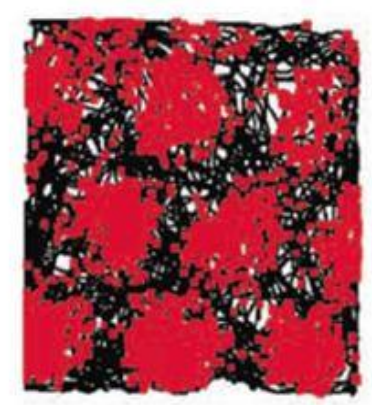

c) Grid cells

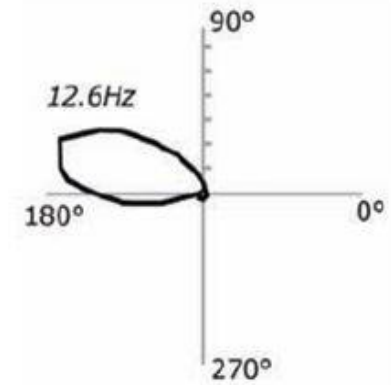

b) Head direction cells

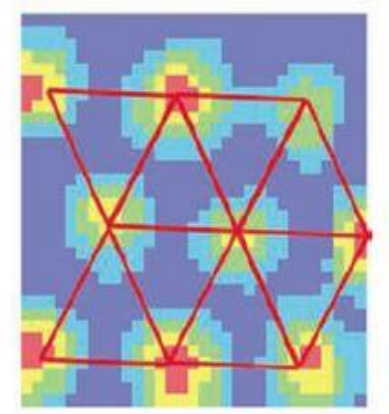

d) Grid cells - firing rate

Figure 5. Selected brain cells involved in navigation. a) Place cells - rat's path is shown as black line; red dots show firing field of observed place cell neurons. b) Head direction cells - plot shows firing rate of an observed neuron for different orientations of the rat's head. c) Grid cells - red dots show firing fields of observed cells. Grid cells have multiple firing fields, which are distributed in a regular triangular grid - diagram (d). Adapted from Doeller, Barry, and Burgess (2012).

head direction cells are neither purely driven by one of the sensory inputs. They are strongly dependent on the vestibular system, but can be 'adjusted' by visual information.

Grid cells are neurons that fire in multiple place fields arranged in triangular shape, or grid. The firing fields of a particular neuron are spaced by the same distance, and same orientation. The specific location of firing field differs between cells. Therefore, just a few grid cells are enough to represent the entire perceived environment. Records from the same grid cell, taken during different experiments (separated in time), confirm that the cell is firing in the same locations. Firing fields are anchored on visual landmarks, and changes of landmarks positions causes corresponding changes in firing locations. Grid cells continue to 
fire even if visual information is unavailable. Grid cells probably influence the place cells. Fyhn, Hafting, Treves, Moser, and Moser (2007) found that by changing the shape or location of the environment, shifts in the vertices of grid cell and remapping of place cells are observed.

Maguire et al. (1998) shown that the more accurate the navigation, the more active the right hippocampus is. Researchers agree that hippocampus plays an important role in navigation, and may be a strong candidate for storing temporal cognitive map. In the same study (Maguire et al., 1998), positive correlation between left frontal brain activity and requirements for strategy switching, in the presence of unexpected obstacles during navigation, has been shown. Frontal brain play important role in planning and decision making (Luria, 1966).

\subsection{Cognitive Processes of Navigation}

Psychology studies on human navigation provide many important and interesting findings, which help to better understand this complex ability. Next, we will present most relevant ones. Locomotion, as one of navigation abilities, should be perceived as mainly visually guided behaviour. Despite of our vestibular and proprioceptive systems, if deprived of sight, humans' locomotion abilities drastically decrease. When people try to walk in a straight line without vision veering can be observed (Kallie, Schrater, \& Legge, 2007; Souman, Frissen, Sreenivasa, \& Ernst, 2009). The debate why this occurs is ongoing, and is not crucial for this thesis. Studies of Böök and Gärling (1981) investigated human performance in estimating distance and direction. Participant had been placed in the dark test room, where he or she saw red light for 5 seconds. Next, after light was turned off, subject was guided to a new position between 1.4 and 11 meters from previous one. In the absence of visual information people perform poorly when estimating the distance and direction to the previously seen light. Error in estimations increased with increasing distance between new and old subject position in the 
room. Those studies indicate that without vision people perform poorly in navigation task, both during locomotion or wayfinding. Next, we will review a few studies which allow us to better understand vision's role in human navigation.

Lee (1974) proposed a theory of optical flow. He argues that locomotion is controlled through temporal brain resources, rather than more complicated spatial concepts. The brain analyses visual input from the eyes to estimate how quickly and in which direction the body is moving in relation to the perceived objects. We use these estimations to make adjustments to our locomotion (e.g. turn, slow down, accelerate etc.). But how human is able to filter out the fact that the eyeballs are moving while the body is moving? For the purpose of this thesis it is sufficient to assume that human interprets itself as a whole body, not an independent moving components (Gibson, 1986). Kearns, Warren, Duchon, and Tarr (2002) in their studies show evidences that information about self-motion is used in privilege to optical flow, when estimating body movements.

While orientating within environment visual landmarks can be very useful. Wikipedia ${ }^{1}$ defines a landmark as "a recognizable natural or artificial feature used for navigation”. In their experiment Lynch (1960) asked people to sketch their hometown. After comparing a large number of sketches, one common element in these sketches Lynch called landmarks - identifiable objects which serve as external reference points. Presson and Montello (1988) define landmarks as "objects that are relatively better known and define the location of other points". In other words, scenes, to be used as landmarks (points of reference), must be scenes that are relatively better known than the other scenes in their neighbourhood. In addition, landmarks are selected along a path, and used during learning and recalling turning points along the path (Allen, 1999). Moreover, the use of landmarks transcends human navigation of physical spaces to include navigation in virtual environments

\footnotetext{
${ }^{1}$ https://en.wikipedia.org/wiki/Landmark, visited 06/06/2017
} 
(Dieberger \& Frank, 1998). In general, landmarks are essential for any spatial reasoning (e.g., for orientation and wayfinding). Also, people use them quite naturally.

Riecke, van Veen, and Bülthoff (2002) showed that in virtual town, participants are able to return to a start position, with an average error of $4^{\circ}$. In contrast, when tested in virtual environment, which contained only 3D uniform abstract objects (for optical flow perception), average error was $18.6^{\circ}$. Foo, Warren, Duchon, and Tarr (2005) investigated the ability to take a shortcut between two virtual locations. Both locations had been experienced independently. When attempting to take a shortcut of just 9.5 meters between two locations in a virtual desert environment, participants were off (on average) by $30^{\circ}$. In contrary, when stable landmarks had been added to the virtual environment, participants were much more accurate, especially in cases when the landmarks were near the goal location. From these results, it may be concluded that people are not very accurate at calculating the distance and direction between locations, which have been experienced separately. In addition, AbuGhazzeh (1996) showed that the uniform visual nature of the space made an environment extremely difficult to learn and navigate - nearly identical appearance of the University buildings and internal floor plans. However, people use visual landmarks to make shortcuts. In another work of Foo, Duchon, Warren Jr, and Tarr (2007), they show that visual landmarks are used during wayfinding, even when seen for the first time. In general, a landmark is not a global characteristic of a particular object or scene, but rather a temporal property perceived by an observer who uses the landmark to memorize an environment and/or to make navigation decision. Together, these studies indicate that people use visual landmarks (when present) instead of other sources of information during wayfinding,

According to traditional view, when we represent large environments (beyond area perceived by our visual system), we use a stable, Euclidean representation of the distances and directions between different landmarks and interesting locations. It is equivalent to 
allocentric view of the environment. However, alternative view assumes that human represent his location by updating current position relatively to individual landmarks. R. F. Wang and Spelke (2000) proposed model, in which human maintains his current position by keeping track of how far he has moved relative to the environment's landmarks. This way of representing the environment is called egocentric view. In traditional view, we remember spatial relation between landmarks; we also keep tracking our distance and direction travelled. If we are lost, we are still able to recall how the landmarks are related to each other. In egocentric view, our current position is updated relatively to landmarks. If we are lost, and become disoriented, our ability to recall spatial relationships between landmarks would be disrupted. Based on previous findings and his own works, Burgess (2006) tried to reconcile the allocentric and egocentric views. In the Chapter 5 (Towards a Novel Cognitive-Inspired Indoor Navigation System), we proposed a two-tier hybrid model in which allocentric and egocentric representation cooperate. Allocentric representation is used to create topological structure of the egocentric representations of an environment.

What is needed for efficient wayfinding during navigation? Cutting (1996) proposes an overall strategy for wayfinding. It can be presented as four subtasks:

- $\quad$ Plot a course (path) through the environment representation.

- $\quad$ Periodically check progress in following the planed path

- $\quad$ Avoid fixed and moving obstacles

- If returning in unfamiliar environment, recognize landmarks from the reverse side, and navigate towards initial location

\subsection{Indoor Localization and Navigation with Mobile Devices}

Navigation on mobile devices is becoming dominant and the number of users is likely to increase in the next few years. Many users benefit from these systems; however, at the same 
time, they have to cope with high cognitive load, accept poor interface design, or too much content (H. Huang, Schmidt, \& Gartner, 2012).

Indoor localization can be provided by various techniques. Vision-based methods require the user to use a camera - e.g., embedded in a cell phone or a wearable (head mounted) device, like Google Glass. While users move through an environment, a camera processes images of the environment, these images are compared against a database of images with assigned (known) location. This process allows to determine users' position and orientation within an environment. (H. Li, Zhang, Wu, \& Mei, 2016; L. Li et al., 2016). The main disadvantage of the vision-based method is the high storage capacity required for storing all the images (or their features) that are paired with the environment structure. Also, often a significant computational power may be needed to complete the image matching process (Hightower \& Borriello, 2001). This requirement is especially challenging to implement, given current computational power of a handheld or wearable devices.

Dead reckoning technique estimates a user's location based on a previously estimated or known position (Harle, 2013). This kind of systems estimates the user's location through the aggregation of odometry readings. However, the inaccuracy and the need to combine it with other techniques are the main downsides of the dead reckoning method. Radiofrequency identification (RFID) method use active (Ding, Yuan, Jiang, \& Zang, 2007), or passive tags (D'Atri et al., 2007). Some solutions use both types of tags (Chumkamon, Tuvaphanthaphiphat, \& Keeratiwintakorn, 2008). Active tags are equipped in a battery and transmit identification signals automatically. These tags have a larger range - when compared to passive tags - which reduces the number of required tags that need to be used. The downside of active tags is the required maintenance. Passive tags do not use a battery to transmit signals, they are powered by the RFID reader. Passive tags are much less expensive, 
but at the same time they have much shorter range, which increases their installation cost more RFID readers are required within an environment.

Moreover, some navigation systems make use of barcodes or quick response (QR) codes, embedded within an environment, to provide navigation instructions through personal digital assistant (Chang, Tsai, Chang, \& Wang, 2007). While navigating through an environment, a user has to scan the codes along the way and they are located based on code's unique IDs. The personal assistant can then provide the user with information about their current location, and inform whether they are moving in the right direction (Chang, Tsai, \& Wang, 2008). This method is relatively cheap, easy to install and maintain. However, the main drawback of this method is that the user has to localize each barcode (or QR code) and scan it - this may be inconvenient and in general will slow down navigation. Moreover, this method is not suitable for users with visual impairments, as they may be unable to see the codes.

Also, the signal strength from the Wi-Fi access point(s) can be also used for localization (Rajamäki, Viinikainen, Tuomisto, Sederholm, \& Säämänen, 2007). This is an example of signal distribution localization. This method compares the signal data sensed at a particular location with a pre-recorded data. However, this technique requires an intense training phase, during which a map of an environment is created - signal strength has to be measured throughout the environment and then stored in a database. When the user is navigating, the closest match is continuously retrieved by comparing received signal data with a pre-created map of an environment.

In general, there are many technologies that support indoor navigation. In the following sections, we present mobile and wearable navigation systems, that use visual input for guidance - as this thesis focuses on vision-based navigation. We also present different 
interaction modalities utilized for indoor navigation. Finally, we present properties of a usercentred wearable navigation system.

\subsection{Different Interaction Modalities for Navigation Systems}

Modern systems for navigation support focus primarily on user-centred communication. Different interaction modalities can be used in different cases, to accommodate different navigation conditions and user needs. The three main modalities of navigation information are: auditory, visual, and tactile.

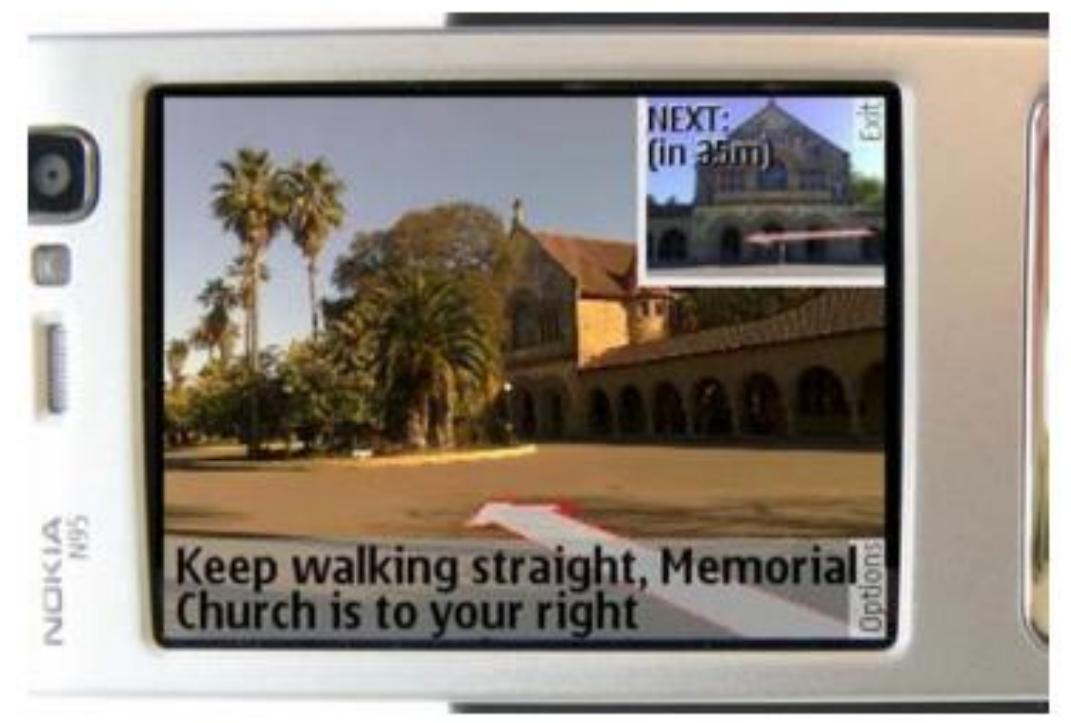

Figure 6. Landmark-based navigation system implemented on a mobile phone (Hile et al., 2008).

An auditory modality may provide instructional or corrective messages with voice information. More dedicated systems, especially for visually impaired, may provide an auditory output which projects a distance from obstacles with high- and low-pitched sounds. It should be considered, that lengthy voice information may overwhelm a user and may impede his or her ability to correctly the navigation instructions. Also, speech-based solutions are language dependent, and therefore, an additional effort is required to incorporate multiple languages into these systems. At the same time, speech-based solutions may be safer than 
using a display, as they require less attention - only the hearing is additionally utilized, and no shifts (between a display and an environment) in visual attention are needed. However, voice-based techniques are not suitable for noisy environments, or when a user has hearing disabilities. An evaluation of only speech-based navigation system has been carried out (Arning, Ziefle, Li, \& Kobbelt, 2012).

Next, providing directions using a display is the most common technique for providing directions. Visual modality can use real (e.g., maps or photos) or simulated imagery (e.g., landmark model), basic graphic icons (e.g., $\rightarrow$ ), or even text (e.g., "Please turn right”). For example, Figure 6 presents a navigation system with route descriptions that include geo-tagged images as additional cues besides textual information (Hile et al., 2008). Using visual modality allows to provide to the user large amount of information in a short amount of time. This may be especially beneficial for individuals with a cognitive disability as they can directly match the navigation guidance (displayed on a screen) with the environment. At the same time, navigation systems with display modality, require users to shift their visual attention between the screen and the environment while following directions. Also, as users' attention is focused on the screen, their safety may be impeded. Moreover, utilizing visual modality is difficult or even impossible for users with visual impairments.

Finally, Figure 7 presents navigation systems, that use tactile feedback to provide navigation information with the use of pulses transmitted by vibration-motors mounted somewhere on user's body, e.g., on a waist belt (Grierson et al., 2009), back array (Ross \& Blasch, 2000), or gloves (Zelek, 2005). This interaction modality can be used even by people with seeing or hearing disabilities. Often, some form of haptification (e.g, changes in signal frequency or intensity) is used to represent a distance or angle between the user and a destination. Haptic interaction modality has far less interference with sensing the environment; however, at the same time it requires training and additional concentration - it 

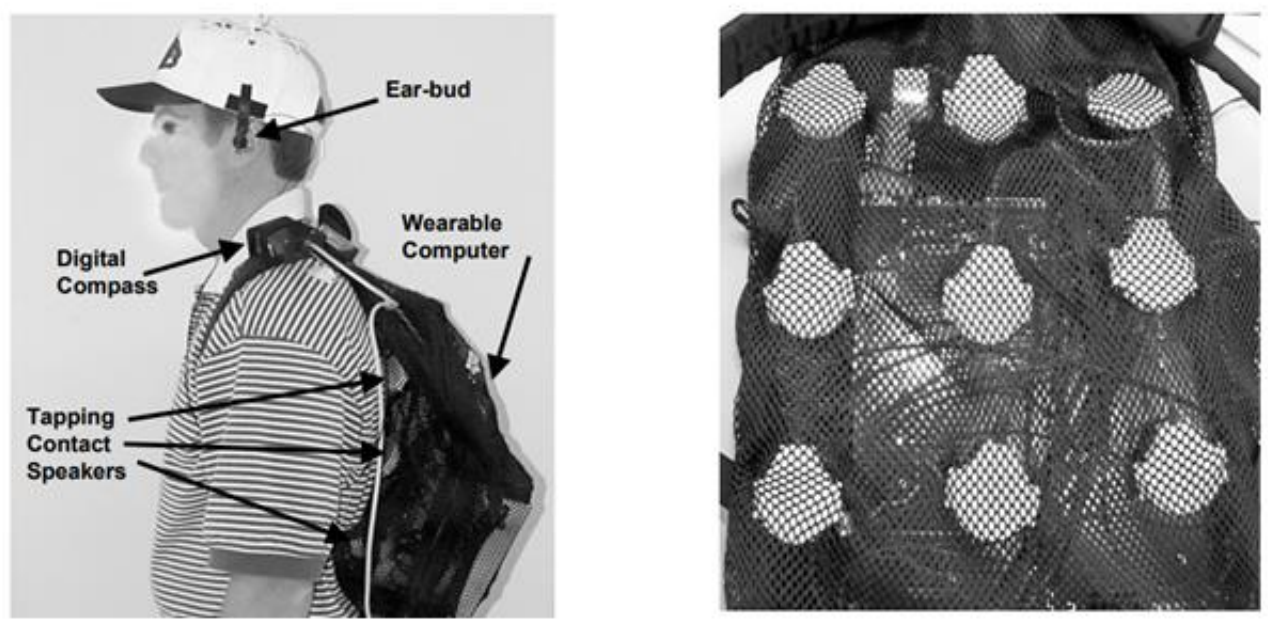

a) b) Back array navigation system with contact speakers

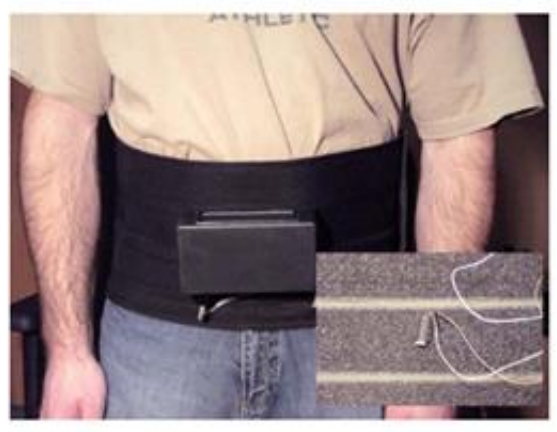

c) Tactile belt

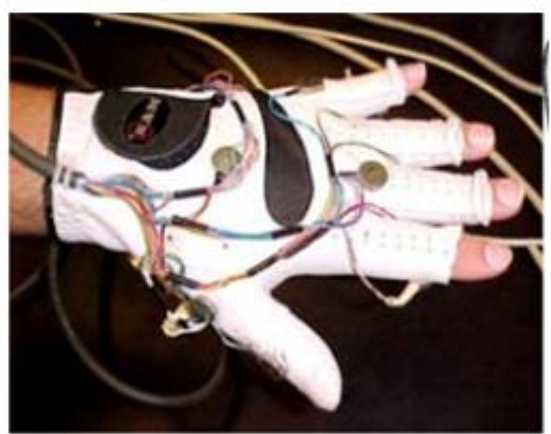

d) Haptic glove

Figure 7. Different types of interaction modalities for navigation. a) and b) present a system which utilizes $3 \times 3$ tapping interface grid - contact speakers. Adapted from Ross and Blasch (2000). c) presents a tactile way-finding belt, which uses vibrating motors (Grierson, Zelek, \& Carnahan, 2009). d) presents a haptic glove, which utilize small vibrotactile motors - adapted from Zelek (2005).

is less natural, for people without disabilities, to receive complicated information through touch. Moreover, systems which utilize haptic modality require additional hardware (e.g., gloves, belt), this may increase their total cost (Srikulwong \& O’Neill, 2010).

\subsection{Handheld and Wearable Indoor Navigation Systems}

Möller et al. (2014) uncovered some disadvantages of augmented reality (AR) interfaces on handheld devices. Users have to hold the devices in an upright position, which is 
inconvenient and may cause fatigue. The typical orientation when holding a mobile phone is $45^{\circ}$ downwards. In this position, mostly the floor is visible to the camera. Therefore, the pose of a device is especially important for vision-based navigation. At the same time, it has been shown that AR solutions provide better user experience than more conventional approaches (Zhong, 2014). A navigation system based on Google Glass (GG), which utilize display, is also considered as an augmented reality (AR) system (U. Rehman \& S. Cao, 2015). Therefore, camera orientation problems related to handheld devices are eliminated with a wearable device equipped with a front-facing camera (e.g., Google Glass), while preserving benefits of AR techniques.

Hub, Diepstraten, and Ertl (2004) present a vision-based system, which uses a handheld camera and Wi-Fi signals for indoor navigation. However, this system requires precisely predefined models of environments to provide guidance, this requirement greatly limits its use in novel environments.

Figure 8 presents the Google Indoor Maps. This navigation system is available for all users who have Android or iOS devices or a web browser. It has over 10,000 floor plans ${ }^{2}$ throughout the world. These indoor spaces include shopping malls, airports, hospitals, etc. Google's indoor navigation algorithm is based on Wi-Fi access points and mobile towers (Base Transceiver Stations) to calculate user's location. Therefore, this system requires additional infrastructure (Wi-Fi access points); and moreover, it requires pre-mapping of an environment - so Wi-Fi and mobile towers signals can be measured at particular locations.

\footnotetext{
${ }^{2}$ As of March 2013.
} 


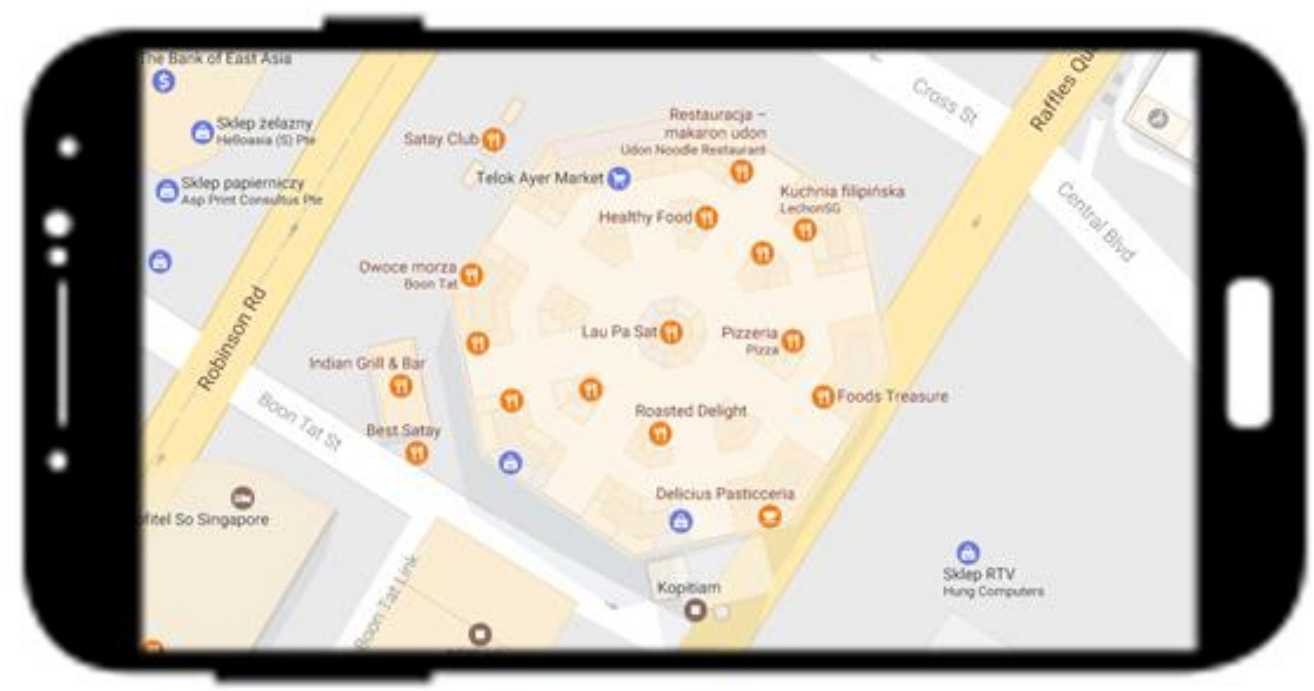

Figure 8. Google Indoor Maps navigation solution allows users to seamlessly switch between outdoor and indoor navigation.

Place Lab indoor navigation system, developed by Intel Research, consists of three elements: RIFDs installed throughout an indoor environment, a database that matches RIFDs with their precise location within the environment, and a user who can estimate his or her location (LaMarca et al., 2005). This solution also requires an additional infrastructure (RIFDs), and precise environment mapping

The NAVVIS localization system uses a database of images of indoor places (Steinbach et al., 2017). These images are used to generate a 3D model of an environment. While using NAVVIS, a user takes a picture of his surroundings and the system computes his or her location and orientation by comparing this picture with the images in the database. This solution requires a large database of images of environments at which we would like to get navigation assistance. Moreover, for a large number of 'mapped' environments (stored in the database), the number of stored images may be vast, which could result in a long computational time needed for image matching process. 


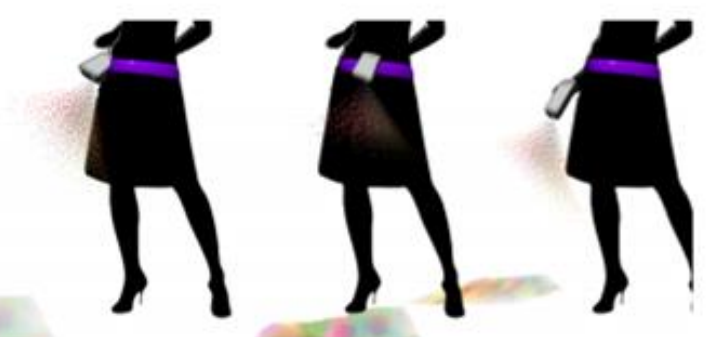

a) Projector at different possible locations on the belt

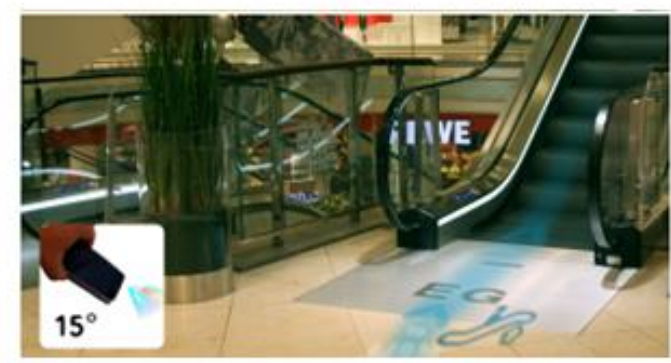

b) Visualization of navigation (mockup)

Figure 9. Navigation system which utilize projector for guidance. The projector displays, in front of a user, navigation path. Adapted from Winkler, Broscheit, and Rukzio (2011).

Furthermore, Figure 9 presents a projector-based navigation system proposed by Winkler et al. (2011). Users wear a mobile phone with a projector at their belt. The projector constantly projects a navigation information in front of them. This solution has many advantages, for example, user has free hands, navigational information is displayed directly on a walking path. However, this solution is not feasible for crowded places, at which projected information will not be clearly visible - most of the time the projector will display information on someone's back.

Wearables, with a display, provide an excellent platform for hands-free, unobtrusive navigation assistance (Joseph et al., 2013; Kalkusch et al., 2002). However, as with any new emerging technologies, we need to understand their limitations, analyse how people interact with them, and address any potential danger that can result (Froehlich, Baillie, \& Simon, 2008). Despite that, there is still a lack of studies on the evaluation of navigation systems based on wearable devices.

Umair Rehman and Shi Cao (2015) investigated the usability of augmented reality navigation prototype implemented on Google Glass (see Figure 10) and a handheld device (i.e., mobile phone). Also, 2D paper map is used for comparison. However, in this study, the authors do not investigate how the navigation system affects users' spatial cognition, nor do 

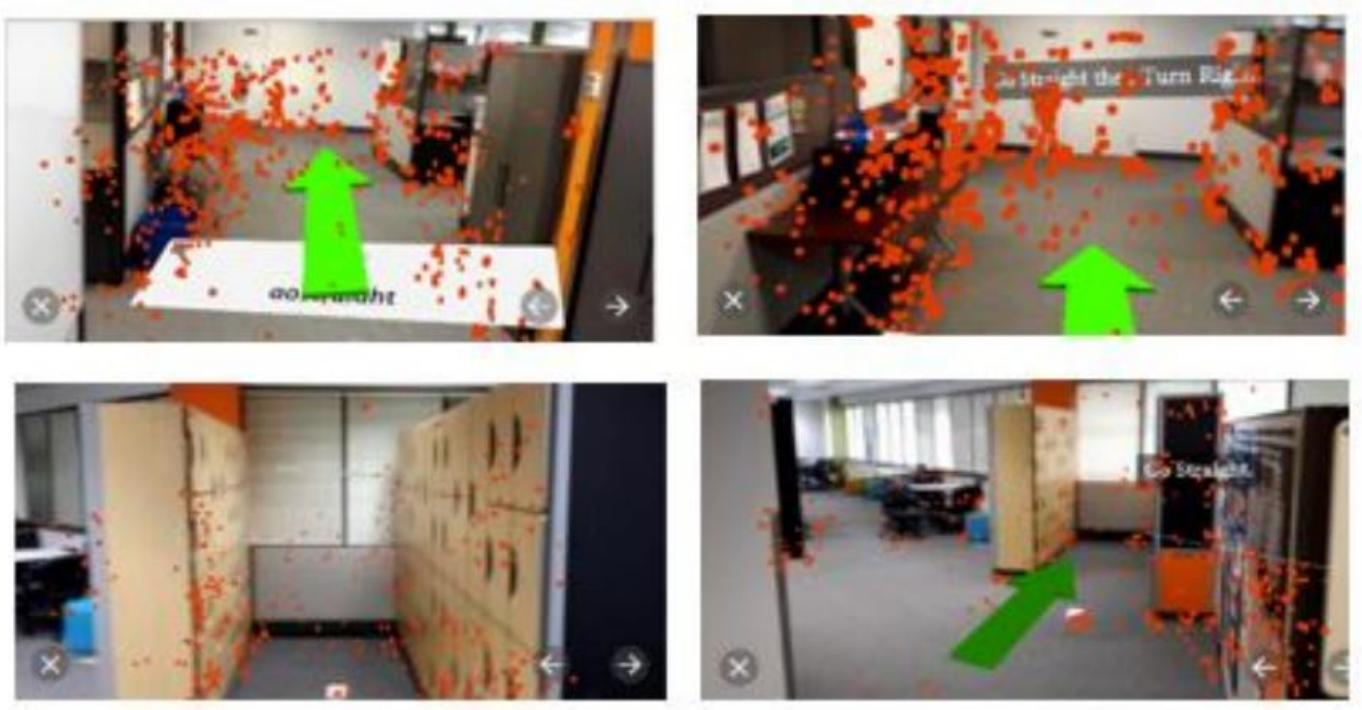

Figure 10. Navigation system that uses vision-based methods for localization orange dots indicate recognized points. Guidance information is provided on the Google Glass display (green arrow). Adapted from U. Rehman and S. Cao (2015).

they analyse the efficiency of Google Glass navigation against a Human Guide (which may be considered as the most effective navigation assistance). Moreover, in the mentioned study, only one interaction modality is evaluated, namely: voice information with additional augmented reality direction (i.e., superimposed direction arrow). In addition, this study uses "real" localization method (i.e., image recognition for localization). Therefore, the localization accuracy variation between Google Glass and the mobile phone might be significant as both have different types of camera and processing power. In addition, the orientation of the camera may be different for each user. These variabilities result in discrepancies between locations at which navigation command is provided for a particular user.

Giannopoulos, Kiefer, Raubal, Richter, and Thrash (2014) that stated the combination of user, navigation instruction, and environmental factors, is more important than any single factor alone. In addition, some studies show that users tend to rate unimodal technology as easier to use (Loomis, Marston, Golledge, \& Klatzky, 2005). Therefore, our research investigates how a wearable, autonomous indoor navigation system affects users' memory 
and spatial abilities, when compared to traditional 2D map navigation and human guide. Moreover, our research examines how a Google Glass based, navigation system with augmented reality feature affects users' navigation abilities, memory, and subjective experience, when compared to human guide. Also, our study investigates which interaction modality (i.e., voice, display, voice+display) should be used in order to achieve human-level, effective, and acceptable assistance for indoor guidance.

Finally, navigation assistance systems may lead to dependence on them, which can reduce users' proactive effort in exploring the environment. Therefore, it may negatively affect an individual's spatial cognition abilities in the long run. In our research, we verify this possibility by evaluating the participants' navigation and backtracking performance, as well as their visual memory. 


\section{Scene Memorability and Visual Memory - Experiments}

\subsection{Navigation Assistance and Scene Memorability - Introduction and} Methodology

Our ability to navigate represents one of the most fundamental and complex humans' cognitive function (Doeller, Barry, and Burgess 2012). In novel environments, we are able to learn fast and assimilate the general and detailed topology of a surrounding (Dudchenko 2010). Our navigation process can be supported by using information from different sources, like: signs, maps, passers, or even technology-based assistance (e.g., digital maps on mobiles). At the same time, landmarks, even when seen for the first time, are used during navigation (Foo et al., 2007). They seem to be crucial to reduce navigation errors (Foo et al., 2005; Riecke et al., 2002). Also, during mobile guidance for older people landmarks can significantly outperform a paper map in a navigation task (Goodman, Brewster, and Gray 2005). Moreover, some mobile navigation systems have the potential to convey landmark knowledge but fail to convey survey knowledge (Krüger, Aslan, and Zimmer 2004). Therefore, motivated by the research on human navigation and image memorability, we designed an experiment to understand which visual scenes we tend to remember during guided navigation. This experiment investigates if different types of navigation assistance (i.e., map, wearable navigation system, human guide) affect memorability of indoor visual scenes. It is of great importance to investigate how wearable navigation assistance affects our spatial abilities. We choose 2D map and human guidance, as they represent well-known navigation aids. However, these two methods are notably different in terms of the cognitive load required during the guidance. To the best of our knowledge, our study is the first experiment designed to investigate how the mentioned types of assistance affect scene memorability. Moreover, we are curious if people remember scenes with navigation information. Finally, we investigate if a scene location can predict how well the scene will be 
memorized. Focusing on indoor navigation for office buildings, our study on scene memorability as reported in this thesis is applicable to scenarios such as navigation in airports, hospitals, schools, shopping malls, etc.

Each participant used all three navigation assistance - one at a time and in a random order-during navigation through three route stages. The participants were asked to remember the path taken, as they will need to backtrack by themselves. The visual memory retention test was carried out after completing the final stage of the navigation route (but before backtracking). The test aims to reveal which visual scenes participant remembered.

\subsubsection{Participants}

20 adults (12 males and 8 females) were recruited. Ages range between 21 and 33 years old (Mean $=27.2$ with the standard deviation of 2.9 years). All participants had normal or corrected-to-normal vision. Each assisted navigation session lasted for about 50 minutes. The participants have no prior knowledge of the building where the experiment was conducted. The building has two towers; each of them consists of 23 levels and each has separate lift lobby. The towers are connected - by office space - on some floors. For first time visitors, it is hard to notice in which tower they are while moving within these levels. The participants visited three different office levels. The navigation route led through one of the levels with connected towers. The environment consists of a typical office space with cubicles, offices, meeting rooms, and pantries.

\subsubsection{Apparatus}

The participants used three different types of navigation assistance: a 2D map (MAP), a wearable virtual navigator (WVN) (L. Li, Wang, Goh, Lim, \& Tan, 2013), and a human guide (HUG). 2D map shows a floor plan, level number, together with the name and marked location of the navigation goal (see an example in Figure 11 (a)). The WVN provides context-aware assistance to guide the participant. It recognizes current location based on 
visual input provided by a wearable camera. The camera was placed on the participant's forehead (see Figure 5 (b)). Current visual scene is compared with the cognitive route model. Once a match is found, specific predefined navigation instruction is provided to a participant. Sample WVN instructions were as followed: "We will go to Bayes at level ten. Please walk through the glass doors.", "Please turn left towards the South Tower gate.", "You are facing south tower gate. Please go ahead to the lift lobby.". The WVN also provides verbal details about the name of a destination, and level at which it is located (e.g., "We are close to Bayes. It is the room on the left side of the junction.”). During the HUG assistance, the experimenter provides verbal directions necessary for reaching a destination (e.g., "Please go straight ahead through the glass doors.", “At the next junction, please turn left.”).

The visual memory retention test was carried out using Samsung Series 7 Slate tablet (1.86 GHz Core i5 processor and 4GB of RAM). The test was conducted with a simple applet. For each presented image, there are two possible answers: 'Yes' - if a participant thinks that

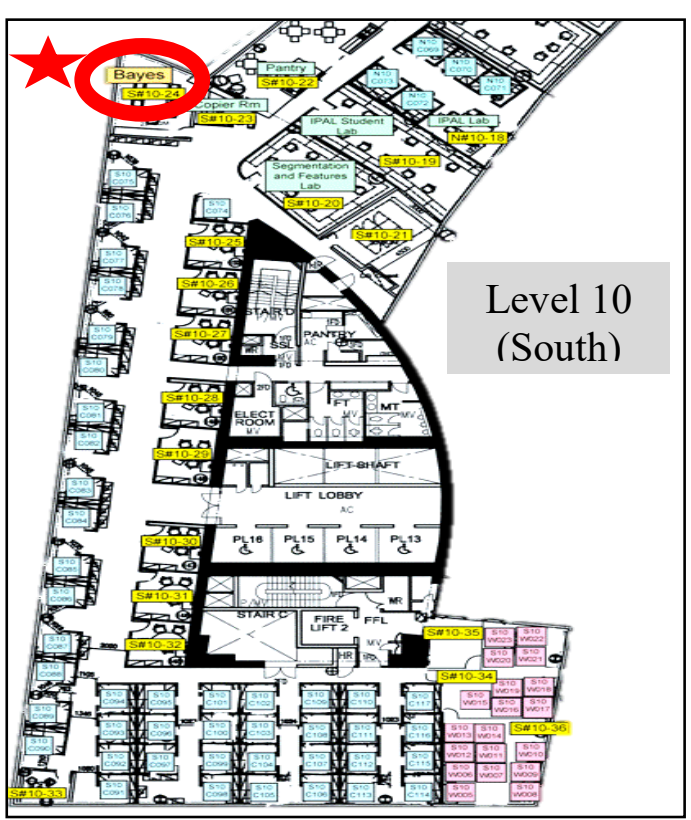

(a)

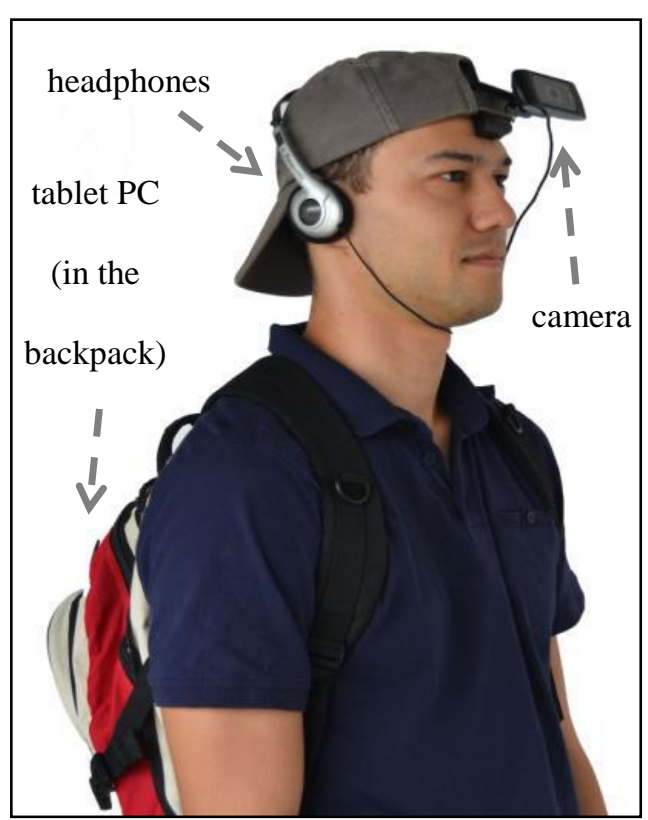

(b)

Figure 11. Two types of navigation assistance: (a) 2D map with marked destination e.g., the first navigation goal (star symbol); (b) wearable virtual navigator Mukawa et al. (2017). 
he or she visited the place presented on the image; and 'No' - if the participant thinks otherwise ("I have not visited the place showed on this image"). Each image is presented for a maximum of 5 seconds. We have chosen 5-second duration based on our preliminary user study. The remaining time is displayed in a form of a progress bar. When no answer is provided within 5 seconds, the system saves it as 'No Response'. The participants performed the visual memory retention test while seated with the tablet held in front of them, or placed on their laps. Therefore, the distance between a participant's eyes and the tablet screen varied. It was in the range between 30 and $60 \mathrm{~cm}$. At these distances, participants viewed the images at $45 \times 18$, and $24 \times 9$ degrees of visual angles, respectively - visual angles in degrees of arc for width $\times$ height of viewed screen.

\subsubsection{Stimuli}

We collected eighty images of indoor scenes. Half of them were taken along the navigation path. Therefore, this subset of images is denoted as the visited-scenes. We chose these spots so that the dataset consists of scenes which are spread equally along the navigation path and scenes which are important for the navigation task (e.g., scenes that contain information about floor number, room name, etc.). The other half were taken in different parts of the same indoor environment, but at locations outside line-of-sight from any point along the navigation path. This subset of images is denoted as the unvisited-scenes. These spots are selected so that the number of scenes at a particular location is balanced. 


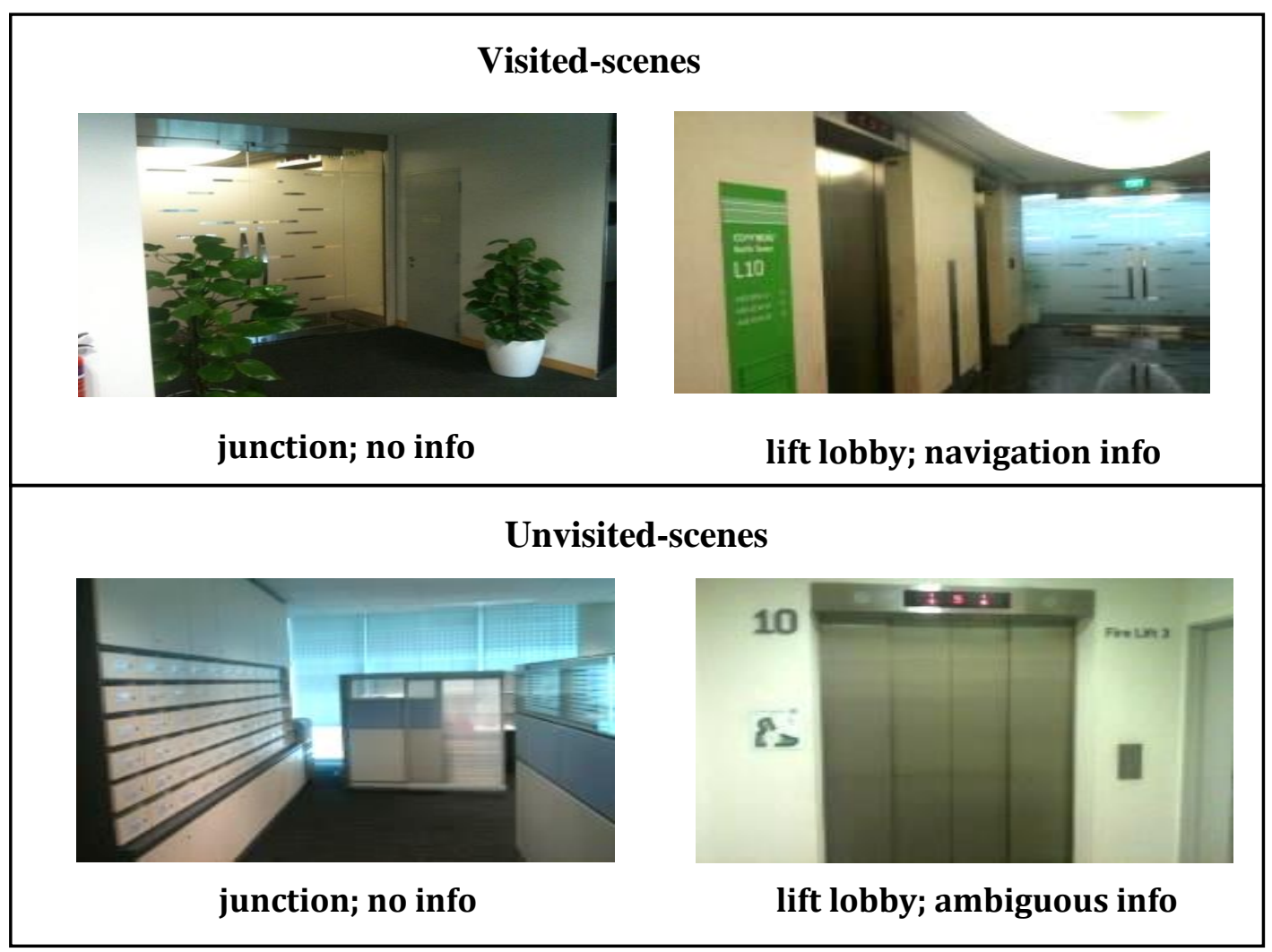

Figure 12. Sample visited-scenes (top row) and unvisited-scenes (bottom row) images with annotations about their location and category of navigation information. Ambiguous info category image presents scene which contains number 10 - indicates floor number. This information can be wrongly interpreted by a participant as a location that he or she visited if mostly semantic information (i.e., level 10) was remembered during navigation task. Adapted from Mukawa et al. (2017).

Within the visited-scenes dataset, we defined two categories, depending on the presence and purpose of the navigation information contained in a scene (see Figure 12). Each image was assigned to one of the categories: "no info" (28 images) or "navigation info" (12 images). The "no info" images, do not contain textual or graphical indicators of location or direction (e.g., the first image on the top row in Figure 12); while the "navigation info" images, do contain information (graphical or textual) about location or direction (e.g. the second image on the top row in Figure 12). We also defined four location categories, depending on the location at which the image was taken: junction (12 images), lift lobby (10 images), corridor (15 images), and target meeting room entrance (3 images). 
Similarly, we defined four location categories for the unvisited-scenes images (see Figure 12): junction (7 images), lift lobby (9 images), corridor (13 images), and others (11 images). Finally, the information categories for the unvisited-scenes images were defined as follow: "no info" (28 images), "navigation info" (7 images), "ambiguous info" (5 images). The scenes, under the "ambiguous info" category, were chosen deliberately to look similar to those scenes encountered by the participants. They contain information which may be easily mistaken for navigation information encountered along the navigation path. For instance, the label " 10 " in the picture of unvisited lift lobby (see Figure 12 bottom row) may be wrongly recognized as the label "L10" in the picture of visited lift lobby (see Figure 12 top row).

\subsubsection{Procedure}

All the participants performed the same task. Instructions provided by the experimenter explained that a participant will wear a camera that is mounted on a cap throughout participation in this research when a participant is asked to locate 3 meeting rooms inside a building using 3 different navigation methods 1) a 2D map with the relevant floor plan, 2) WVN that provides audio-instructions via earphones, 3) HUG that provides audioinstructions. The participants were also instructed to remember the navigation path, as they will need to backtrack without any assistance. The navigation path has three sections:

- Section one: from the main building entrance to the Bayes meeting room;

- Section two: from the Bayes meeting room to the Faraday meeting room;

- Section three: from the Faraday meeting room to the Spectrum meeting room.

All the meeting rooms are located at different levels. The order of the navigation sections was identical across all test sessions, namely, Section one $\rightarrow$ Section two $\rightarrow$ Section three. Participants used different navigation assistance (i.e., MAP, WVN, HUG) at different sections of the navigation path. A participant used each navigation assistance mechanism only once, assigned in different sequence, and counter-balanced for the order of usage - this 
is to control that each navigation assistance was used the same number of times; to cancel out the learning effect and the effect of task difficulty

The time needed to find subsequent destinations was recorded. The visual memory retention test was carried out at the final destination (i.e., Spectrum meeting room), prior to the backtracking task. Before the test, a participant was asked to fill up a few questionnaires that were not related to the study. This procedure took around 3-5 minutes. We assume that the visual memory retention test examines the participant's long-term visual memory (Atkinson \& Shiffrin, 1968).

During the visual memory retention test, 80 images of scenes - in a random order were shown to the participants. For each presented scene, the participant needs to decide (by pressing either 'Yes' or 'No' button) if he or she had seen the scene along the travelled path. Based on the data collected from the visual memory retention test, we compute a memorability score to each image from the visited-scenes dataset. The scene memorability score is defined as the percentage of correct recognitions (i.e. true positive) obtained by all participants; whereas, false positive (FP) score - assigned for each unvisited-scenes image is defined as the percentage of false recognitions (i.e., a participant marked the unvisitedscenes image as the visited-scenes image).

After completing the visual memory retention test, the participant was asked to backtrack to the starting location using the taken path (i.e., Spectrum $\rightarrow$ Faraday $\rightarrow$ Bayes $\rightarrow$ main building entrance). During backtracking, no assistance was provided. The time needed for backtracking was recorded.

\subsubsection{Hypotheses}

In this section, we present hypotheses related to our research goals. We present assumptions on how different types of navigation assistance, and associated cognitive loads, influence scene memorability during navigation; how the presence of navigation information makes a 
particular scene more memorable; and how a location of a scene affects its memorability. For the purpose of this study, we refer to the cognitive load as the total amount of mental effort required from the participant during the navigation task (Xu et al., 2014).

Our first hypothesis states that the participants should remember more scenes while using a 2D map than while being guided by the wearable assistance, or by a human. In general, people who used a 2D paper map to learn a new environment perform better during the route knowledge test, than people who used technology-based assistance (Münzer, Zimmer, Schwalm, Baus, \& Aslan, 2006). Moreover, a map provides a holistic view of the entire environment, which leads to better spatial performance (Willis, Hölscher, Wilbertz, \& $\mathrm{Li}, 2009)$. Also, navigation with the use of a $2 \mathrm{D}$ map requires from a user to mentally associate the navigation information from allocentric map representation to egocentric one, and vice versa. This process involves more attention during observation of an environment (Liu et al., 2009). Therefore, we assume that if the participants pay more attention to the environment, they should be able to remember more scenes.

Next, we expect that the participants will memorize more visual scenes while being guided by a human than while using wearable assistance. Both types of assistance provide just-in-time information and require less effort from the participants - compared to the $2 \mathrm{D}$ map. However, earlier experimental evaluation in similar settings shows that users' cognition load was higher when they used the wearable virtual navigator (WVN) than when they were guided by a human usher (Xu et al., 2014). Therefore, even if wearable assistance and human guidance provide a similar form of assistance, higher cognitive load - associated with the WVN assistance - may reduce participants' visual memory performance.

Our second hypothesis is concerned with the effect of navigation information contained within scenes on the memorability of those scenes. Human visual attention shows higher attractiveness to text regions than to other regions of the real world scenes (H.-C. 
Wang, Lu, Lim, \& Pomplun, 2012; H.-C. Wang \& Pomplun, 2012). Much navigation information within the encountered places is in the form of text. Therefore, we assume that scenes which provide navigation information will be more memorable than scenes without such information.

Our third hypothesis predicts how a location of a particular scene impacts its memorability. During indoor navigation, humans make use of labels to share common concepts of the existence of junctions, corridors, lift lobbies, etc. (Torres-Méndez \& Cervantes-Jacobo, 2012). In the context of our study, we expect that people will memorize more scenes located in lift lobbies, or in front of the meeting rooms than scenes located at junctions or along corridors. This hypothesis is partially based on the fact that most lift lobby scenes contain navigation signs. Also, participants may remember lift lobby scenes better as they have been waiting for lifts, while for other categories they are just passing by. In addition, during exploration of an environment, people do not acquire more navigation knowledge at intersections than between them (Gale, Golledge, Pellegrino, \& Doherty, 1990). Meeting room entrances should be memorized by the participants as they are the intermediate and final goals of the navigation task.

We summarize our hypotheses in Table 1. The number of remembered scenes, obtained from the visual memory retention test, is our dependent variable. The hypotheses are formulated with respect to three independent variables: (1) type of a navigation assistance, (2) significance of an information contained in a visual scene, and (3) a location of a particular visual scene. The " $\mathrm{X}=\mathrm{Y}$ " formula means that there is no statistical significance difference in the number of remembered scenes under the respective conditions. The " $\mathrm{X}>\mathrm{Y}$ " formula means that the number of remembered scenes is significantly higher in condition $\mathrm{X}$ than in condition Y. 
Table 1. Scene memory hypotheses with respect to our variables. (MAP - 2D map, WVN - Wearable Virtual Navigator, HUG - Human guide).

\begin{tabular}{c|c} 
Independent Variable & Hypotheses on Scene Memorability \\
\hline Navigation Assistance & MAP $>$ HUG $>$ WVN \\
\hline Contained Information & navigation info $>$ no info \\
\hline Location & lift lobby $=$ room entrance $>$ junction $=$ corridor \\
\hline
\end{tabular}

\subsection{Navigation Assistance and Scene Memorability - Results}

In this section, we present experimental results collected from twenty participants. All of them successfully completed the experiment, by finding the way to three different meeting rooms (i.e., Bayes, Faraday, Spectrum) during guided navigation. Also, all participants were able to backtrack by themselves. The mean time required for locomotion from the main building entrance to the final location (i.e., Spectrum meeting room) was $7 \min (S D=1 \mathrm{~min})$. We found no difference in the navigation times between different types of navigation assistance $[F(2,59)=0.03, p=.974]$. The mean time needed for backtracking was 5 min 40 $\sec (S D=1 \mathrm{~min})$, it indicates that participants were able to find the correct path efficiently. Also, no difference in the backtracking performance was found between sections at which a particular navigation assistance was used $[F(2,59)=0.32, p=.731]$.

After analysis of the visual memory test data, we noticed that the number of "No response" answers accounted for approximately $1 \%$ of all the answers. This is considered to be a marginal amount. Therefore, for simplicity, "No response" was considered as a "No" answer.

The mean memorability score, for all 40 visited-scenes images, was $47.9 \%$ ( $S E=$ $4.3 \%$ ), which is at chance level. The mean false positive (FP) score was $26.3 \%$ ( $S E=4.7 \%$ ), which is significantly lower than the chance level. Although the mean memorability score (47.9\%) seems to be obtained by chance two indicators show otherwise. Firstly, the 
discriminability index ${ }^{3}\left(d^{\prime}\right)$, between TP and FP answers, is equal to 2.18 . This indicates that the participants are sensitive to the difference between the TP and the FP answers - their answers are not at chance level. Secondly, by randomly splitting the participants into two independent halves (10 participants in each group), and comparing how well the memorability scores of the first half match the memorability scores of the second half, we evaluate performance in human consistency (Isola, Xiao, et al., 2011). Over one hundred of these random splits ${ }^{4}$, the mean value of Spearman's rank correlation $(\rho)$ is $0.75(S D=.054)$. This shows that the participants tend to remember the same scenes. In conclusion, discriminability index and human consistency values indicate that the obtained memorability scores are adequate measures of how well the participants memorized particular scenes answers, of a particular participant, are not at a chance level, and participants tend to memorize the same visual scenes

\subsubsection{Memorability of Scenes with Different Types of Navigation}

\section{Assistance}

To check how different navigation assistance mechanisms impact memorability, we computed the method-specific memorability score for each of the visited-scenes images. This score is computed for each scene with respect to the used navigation assistance. The methodspecific, mean memorability scores for all visited-scenes images are as follow (see Figure 13) $\mathrm{MAP}=44.7 \%(S E=4 \%) ; \mathrm{WVN}=50.6 \%(S E=4.1 \%) ; \mathrm{HUG}=45.5 \%(S E=3.7 \%) . \mathrm{A}$ one-way within-subject ANOVA show no significant effect of navigation assistance on scene memorability during guided navigation $[F(2,19)=0.66, p=.525]$. Based on these results, we conclude that different types of navigation assistance did not impact the memorability of encountered visual scenes. This finding does not support our hypothesis. Scene memorability

\footnotetext{
${ }^{3}$ The distance between the two means of the distributions measured in terms of their standard deviations (Macmillan \& Creelman, 2004).

${ }^{4}$ Repeat 100 times: randomly split the participants into two independent halves and compute correlation of memorability scores between those halves. Report the mean correlation score and $S D$.
} 


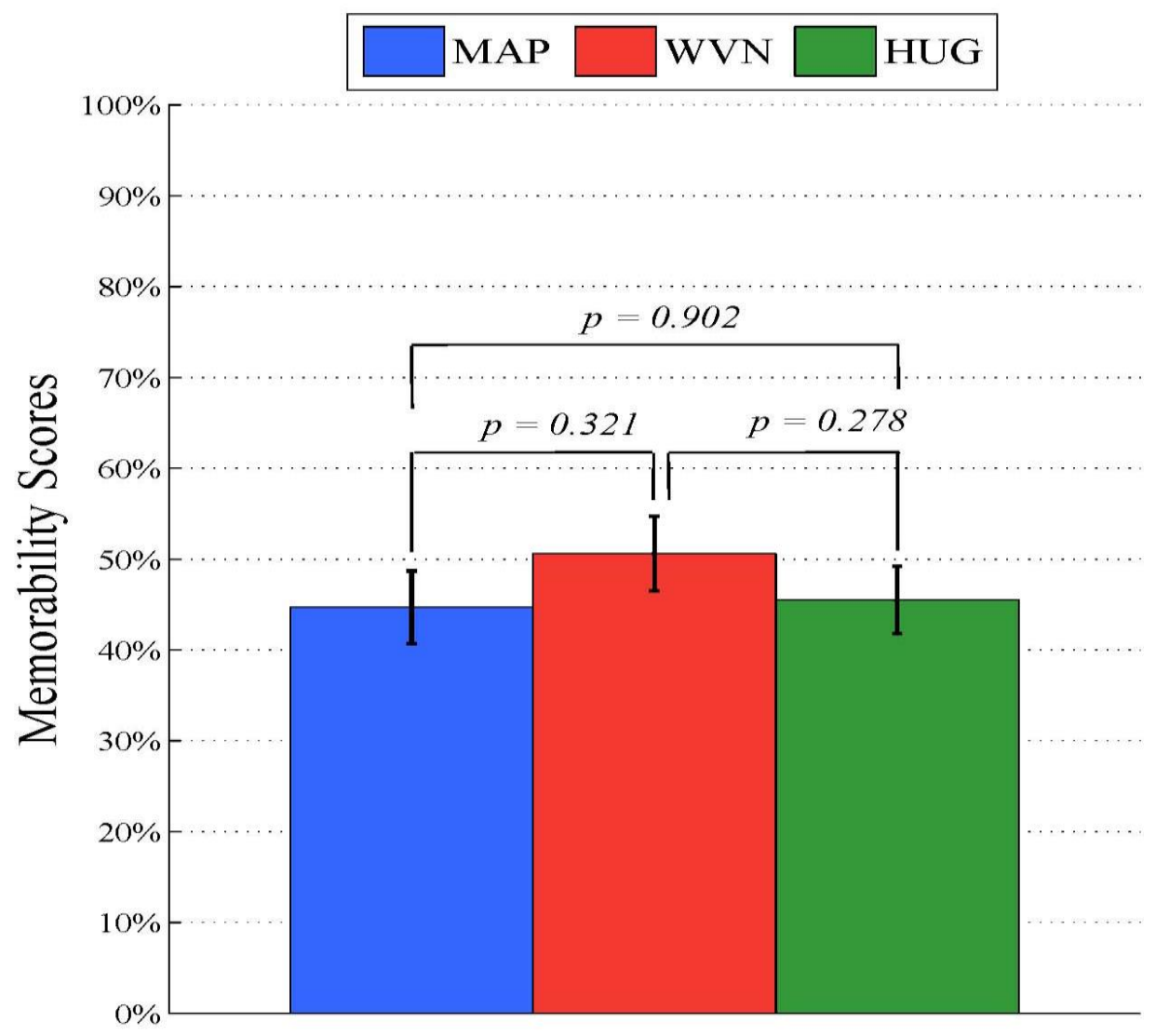

Navigation Assistances

Figure 13. Scene memorability scores for different types of navigation assistance. MAP 2D map; WVN - Wearable Virtual Navigator; HUG - Human Guide. Error bars show the standard error. Sample size $n=20$. Adapted from Mukawa et al. (2017).

is not higher with the use of 2D map as an assistance when compared to WVN and HUG. Also, the participants, guided by human, do not remember more scenes than those guided by wearable assistance. However, the lack of a statistically significant difference between different types of navigation assistance might be caused by the small number of participants.

An earlier experiment evaluated the users' cognitive load - how much mental effort the subject has to make to complete the task - experienced while using different types of navigation assistance compared in our study (Xu et al., 2014). The mean scores of the cognitive load, obtained from 7 levels Likert scale ${ }^{5}$, were as follow: MAP $=4.71$; WVN $=$

\footnotetext{
${ }^{5}$ An example question: 'It required much mental load to find the place using the navigation guide', possible Likert scale answers are numbered from 1 to 7 , where 1 is denoted as 'Not at all', 4 is denoted as 'Moderately so', and 7 is denoted as 'Very much so'.
} 
3.69; HUG $=2.81$. These results indicate that participants experienced significantly lower cognitive load when being guided by human, than when using $2 \mathrm{D}$ map, or wearable assistance [(MAP, HUG): $p<.001$; (WVN, HUG): $p=0.02$ ]. Also, cognitive load was significantly lower for wearable assistance than for 2D map [(MAP, WVN): $t(11)=-5.65$ $p<.001]$. These results show that people report different cognitive load-mental effort, required to finish the navigation task-for different types of assistance. However, in our experiment, scene memorability is unaffected by the type of assistance and corresponding cognitive load. This may indicate dissociation of spatial working memory, reported as cognitive load, and visual memory.

In general, types of navigation assistance as compared in the study - and the corresponding cognitive loads - do not affect memorability of encountered visual scenes. The participants remembered similar number of places. Also, results show a high correlation between memorized scenes.

As different types of assistance do not affect scene memorability, the remaining results are presented without distinguishing which of them was used. Hence, from this point onward, memorability score of a scene is reported as the average from all the memory tests.

\subsubsection{Memorability of Scenes with Different Navigation Information,} and at Different Locations

To investigate how information contained within a visual scene, and location of a scene, affects its memorability, we used factorial design. The two factors are: location (e.g., junction, lift lobby) and contained information (e.g., no info, navigation info). In the Stimuli section we presented two information categories for the visited-scenes images: "no info", and "navigation info"; and three categories for the unvisited-scenes images: "no info", "navigation info", and "ambiguous info". We also introduced four location categories for visited-scenes: junction, lift lobby, corridor, and target meeting room entrance; and four 
location categories for unvisited-scenes: junction, lift lobby, corridor, and other. Table 2 presents mean memorability score, standard deviation, and number of images for different groups under visited-scenes dataset. Table 3 presents mean false positive score, standard deviation, and number of images for different groups under unvisited-scenes dataset.

A 2x4 within subjects ANOVA, for visited-scenes, shows interaction between contained information and location factors $[F(3,133)=12.1, p<.001]$. Also $3 \times 4$ within subjects ANOVA, for unvisited-scenes, shows interaction between the factors $[F(3,171)=$ $82.2, p<.001]$. Therefore, we will analyse the results with respect to this confounding effect.

Table 2. Mean memorability score (mean $M$ ), standard deviation, and number of images for each group of visited-scenes dataset.

\begin{tabular}{|c|c|c|c|c|c|}
\hline \multirow{2}{*}{\multicolumn{2}{|c|}{ Visited-scenes }} & \multicolumn{4}{|c|}{ Locations } \\
\hline & & junction & lift lobby & corridor & meeting room \\
\hline \multirow{2}{*}{ 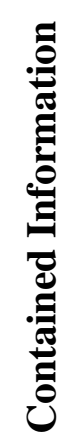 } & $\begin{array}{l}\stackrel{\bigcirc}{\leftrightarrows} \\
\stackrel{0}{\Xi}\end{array}$ & $\begin{array}{c}\text { mean } M=40.1 \% \\
S D=14.2 \% \\
11 \text { images }\end{array}$ & $\begin{array}{c}\text { mean } M=33.3 \% \\
\qquad S D=29.8 \% \\
3 \text { images }\end{array}$ & $\begin{array}{c}\text { mean } M=35.4 \% \\
\qquad S D=16.5 \% \\
14 \text { images }\end{array}$ & NO image \\
\hline & 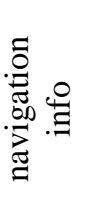 & $\begin{array}{c}\text { mean } M=90 \% \\
S D=30 \% \\
1 \text { image }\end{array}$ & $\begin{array}{c}\text { mean } M=80 \% \\
S D=13.1 \% \\
7 \text { images }\end{array}$ & $\begin{array}{c}\text { mean } M=30 \% \\
S D=45.8 \% \\
1 \text { image }\end{array}$ & $\begin{array}{c}\text { mean } M=63.3 \% \\
S D=25.6 \% \\
3 \text { images }\end{array}$ \\
\hline
\end{tabular}


Table 3. Mean false positive score (mean FP), standard deviation, and number of images for each group of unvisited-scenes dataset.

\begin{tabular}{|c|c|c|c|c|c|}
\hline \multirow{2}{*}{\multicolumn{2}{|c|}{ Unvisited-scenes }} & \multicolumn{4}{|c|}{ Locations } \\
\hline & & junction & lift lobby & corridor & other \\
\hline \multirow{3}{*}{ 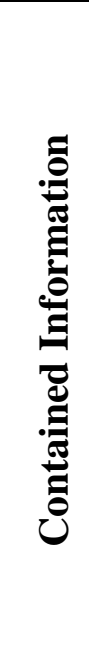 } & $\begin{array}{l}\stackrel{0}{\Xi} \\
\stackrel{8}{g}\end{array}$ & $\begin{array}{c}\text { mean } F P=29.3 \% \\
\qquad D=18.9 \% \\
7 \text { images }\end{array}$ & $\begin{array}{c}\text { mean } F P=5 \% \\
S D=21.8 \% \\
1 \text { image }\end{array}$ & $\begin{array}{c}\text { mean } F P=32.3 \% \\
\qquad D=12.4 \% \\
11 \text { images }\end{array}$ & $\begin{array}{c}\text { mean } F P=5 \% \\
S D=8.9 \% \\
9 \text { images }\end{array}$ \\
\hline & 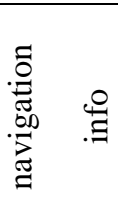 & NO image & $\begin{array}{c}\text { mean } F P=8.8 \% \\
S D=14.3 \% \\
4 \text { images }\end{array}$ & $\begin{array}{c}\text { mean } F P=7.5 \% \\
S D=17.9 \% \\
2 \text { images }\end{array}$ & $\begin{array}{c}\text { mean } F P=70 \% \\
S D=45.8 \% \\
1 \text { image }\end{array}$ \\
\hline & 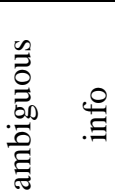 & NO image & $\begin{array}{c}\text { mean } F P=80 \% \\
S D=24.5 \% \\
4 \text { images }\end{array}$ & NO image & $\begin{array}{c}\text { mean } F P=0 \% \\
S D=0 \% \\
1 \text { image }\end{array}$ \\
\hline
\end{tabular}

\subsubsection{The Effect of Navigation Information on Scene Memorability}

Figure 14 presents memorability (i.e., true positive scores) and false positive (FP) scores of scenes that contain different information at different locations categories. We have found a significant difference between visited-scenes that contain or do not contain navigation information $[t(19)=-11.33, p<.001]$. The visited-scenes that contain navigation information and that are located at junctions or lift lobbies show significantly higher memorability scores than scenes without such information assistance [junctions(No Info, Navigation Info): $t(19)=$ -7.43, $p<.001$; lifts(No Info, Navigation Info): $t(19)=-6.72, p<.001]$. The difference in memorability score for scenes located at corridors is not significant [corridors(No Info, Navigation Info): $t(19)=0.52, p=.61$ ]. For the meeting room entrances category, all images contain navigation information. Therefore, it is impossible to quantitatively check if these scenes are more memorable than scenes without navigation information. However, we argue 

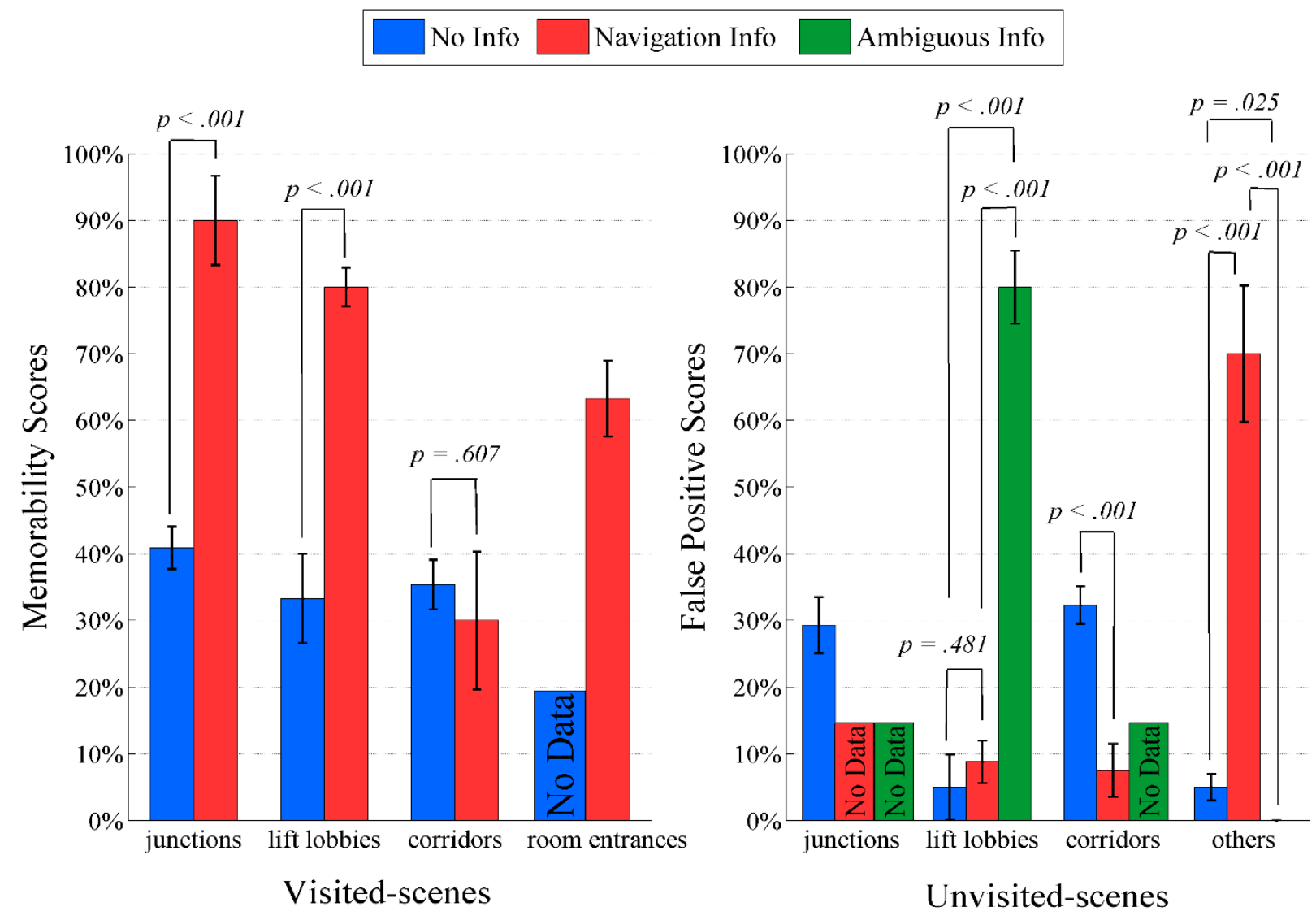

Figure 14. Results of information categories at different locations. Error bars show standard error. Sample size $n=20$. Adapted from Mukawa et al. (2017).

that scenes located at meeting room entrances, which contain navigation information, are more memorable than scenes without this kind of information.

These findings indicate that participants, in general, remembered scenes that contain navigation information, which supports our hypothesis. Those scenes with navigation information should be more memorable than scenes that do not contain this information.

Next, we analysed false positive (FP) answers for the unvisited-scenes images (see Figure 14). We have found a significant difference between unvisited-scenes that contain particular navigation information [(no info, navigation info, ambiguous info): $F(2,19)=81.51$, $p<.001]$. Scenes located at lift lobbies, which contain ambiguous information were most often incorrectly identified as visited places $($ Mean $=80 \%, S E=5.5 \%)$. False positive scores of these scenes was significantly different from scenes which do not contain any information, or 
which contain navigation information [lifts(No Info, Ambiguous Info): $t(19)=-11.05$, $p<.001$; lifts(Navigation Info, Ambiguous Info): $t(19)=-12.9, p<.001]$. The participants made similar number of mistakes when identifying lift lobbies scenes without, and with navigation information [lifts(No Info, Navigation Info): $t(19)=-0.72, p=.48$ ]. Moreover, corridors scenes, which do not contain any information, were incorrectly recognized as visited-scenes more often than corridors scenes with navigation information [corridors(No Info, Navigation Info): $t(19)=4.44, p<.001]$. Surprisingly, scenes with the navigation information under "other" location category are more likely to be incorrectly recognized than scenes with ambiguous information, under the same category [other(Navigation Info, Ambiguous Info): $t(19)=6.66, p<.001]$. In addition, navigation information scenes are also incorrectly recognized more often than scenes with no information [other(No Info, Navigation Info): $t(19)=-6.53, p<.001]$. Finally, scenes with no information, similarly to ambiguous info scenes, are most often correctly recognized as unvisited places [other(No Info, Ambiguous Info): $t(19)=2.44, p=0.25]$. For the unvisited-scenes that are located at junctions, we do not have enough data to provide quantitative analysis.

In general, these results indicate that the participants encoded and memorized navigation information obtained from the encountered scenes. However, the participants did not remember visual details of these scenes, which is indicated by the high false positive score of unvisited lift lobbies scenes with ambiguous info.

\subsubsection{The Effect of Scene Location on Its Memorability}

Figure 15 presents memorability (i.e., true positive scores) and false positive (FP) scores of scenes at different locations for different information categories. In general, we have found a significant difference, in scene memorability, between different visited locations $[F(3,19)=$ 17.03, $p<.001]$.Memorability scores are similar for scenes with no navigation information, which are located at junctions, lift lobbies, or corridors $[F(2,58)=0.64, p=.53]$. However, 

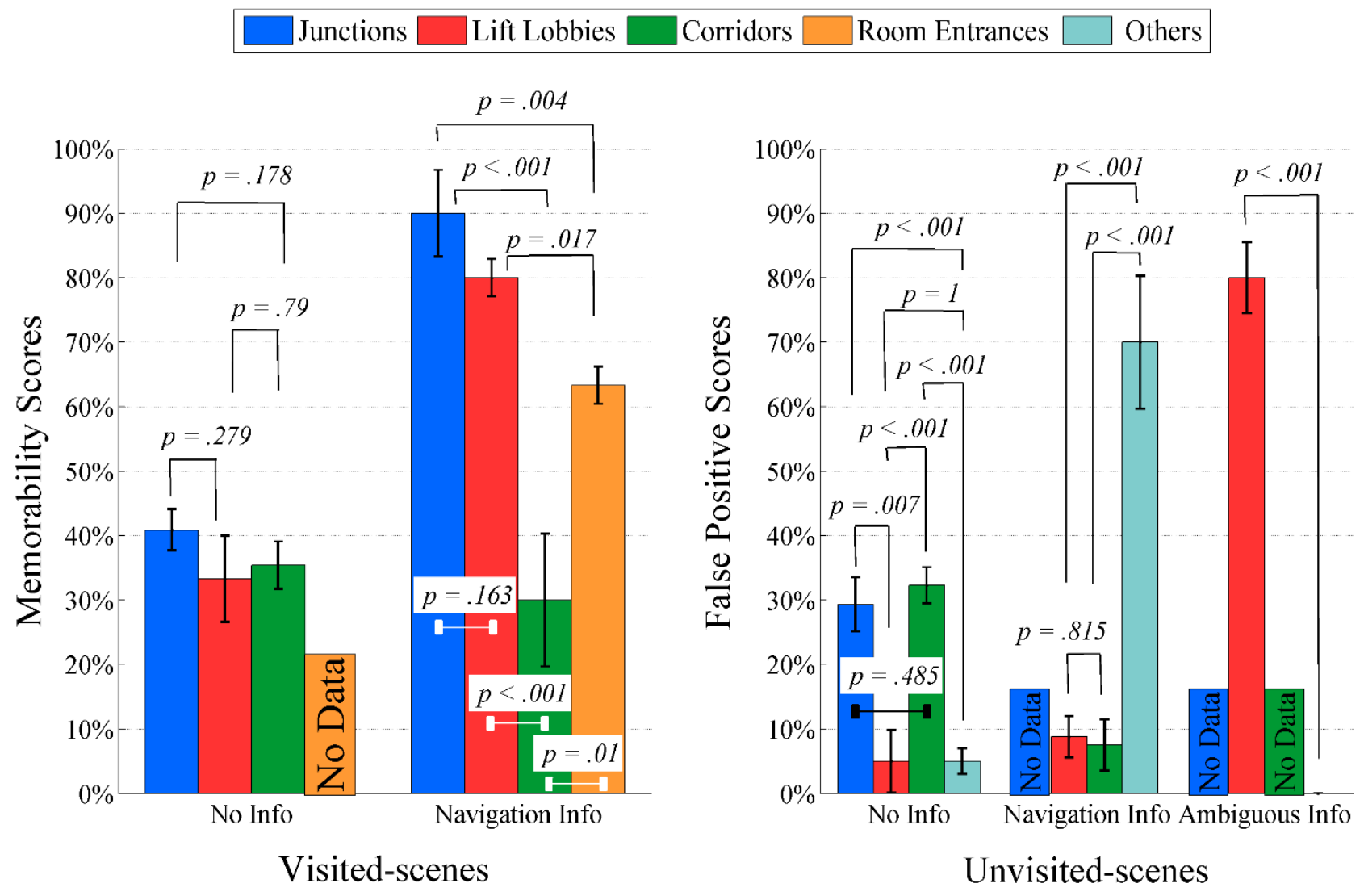

Figure 15. Results of location categories for different information categories. Error bars show standard error. Sample size $n=20$. Adapted from Mukawa, Tan, Lim, Xu, and Li (2017).

memorability scores of scenes, which contain navigation information (navInfo), differ across locations. Junctions and lift lobbies scenes are significantly more memorable than corridors, and meeting room entrances scenes [navInfo(Junctions, Corridors): $t(19)=4.49, p<.001$; navInfo(Lift Lobbies, Corridors): $t(19)=4.63, p<.001$; navInfo(Junctions, Room Entrances): $t(19)=3.24, p=.004$; navInfo(Lift Lobbies, Room Entrances): $t(19)=2.62, p$ $=.017]$. Also, scenes of meeting room entrances are significantly more memorable than scenes of corridors [navInfo(Corridors, Room Entrances): $t(19)=-2.87, p=.01$ ]. Finally, there is no significant difference between memorability of scenes, which provide navigation information, and are located at junctions, or lift lobbies [navInfo(Junctions, Lift Lobbies): $t(19)=1.45, p=.16]$. 
These results partially support our hypothesis that participants would memorize more scenes at lift lobbies and in front of target meeting rooms than scenes at corridors. However, scenes at lift lobbies and room entrances are not more memorable than scenes at junctions. These might be partially explained by the fact that there was only one scene with navigation information located at a junction. While, there were seven scenes, with navigation information, located at lift lobbies.

Next, we analysed false positive (FP) scores for the unvisited-scenes images (see Figure 15). We checked if the participants incorrectly identified scenes from a particular category, or if their mistakes are evenly distributed across all categories. Scenes located at lift lobbies, which contain ambiguous information (ambInfo), are incorrectly recognized as visited-scenes more often than scenes under the others category [ambInfo(Lift Lobbies, Other): $t(19)=14.24, p<.001]$. Also, scenes from others category, which contain some navigation information, are more prone to be incorrectly identified, when compared to scenes with navigation information located at lift lobbies or corridors [navInfo(Lift Lobbies, Other): $t(19)=-5.31, p<.001$; navInfo(Corridors, Other): $t(19)=-5.48, p<.001]$. There is no significant difference in between FP scores of lift lobby and corridors scenes, which contain navigation information [navInfo(Lift Lobbies, Corridors): $t(19)=0.24, p=.815$ ]. Finally, scenes that do not provide navigation information (noInfo) and are located at junctions or corridors, are incorrectly recognized as encountered places similar number of times [noInfo(Junctions, Corridors): $t(19)=-0.71, p=.49]$. Moreover, junctions scenes are incorrectly recognized more often than scenes at lift lobbies, or other places [noInfo(Junctions, Lift Lobbies): $t(19)=4.01, p=.007$; noInfo(Junctions, Other): $t(19)=$ $5.95, p<.001]$. Also, scenes of corridors are more often wrongly identified when compared to scenes of lift lobbies, or other places [noInfo(Lift Lobbies, Corridors): $t(19)=-4.91, p<$ .001 ; noInfo(Corridors, Other): $t(19)=8.82, p<.001$ ]. Finally, results show no difference in 
false positive scores between scenes with no navigation information, at lift lobbies and other places [noInfo(Lift Lobbies, Other): $t(19)<.001, p=1]$.

In conclusion, results indicate that the participants remembered scenes which provide navigation information, and which are located at important spots, such as: junctions, lift lobbies, or room entrances. When faced with images of unvisited scenes, the participants made most mistakes if a particular scene contained ambiguous information, which might be wrongly interpreted as information encountered along the navigation path.

\subsection{Navigation Assistance and Scene Memorability - Discussion}

This study investigates which visual scenes we memorize during navigation. In particular, we explore how different types of navigation assistance, and associated cognitive loads, affect scene memorability during guided indoor navigation. We compared three types of assistance: the $2 \mathrm{D}$ map, the wearable virtual navigator, and the human guide.

\subsubsection{Navigation Assistance Do Not Affect Scene Memorability}

In our hypotheses, we think that people should remember the largest number of visual scenes while using 2D map for navigation, and moreover the participants will memorize more visual scenes while being guided by a human than while using wearable assistance. The results do not confirm our predictions. Scene memorability scores $(\mathrm{MAP}=44.7 \%$; $\mathrm{WVN}=50.6 \%$; HUG $=45.5 \%$ ) show that the participants remembered almost equal number of visual scenes while using different types of navigation assistance. Moreover, a one-way within-subject ANOVA shows no significant difference in scenes recognition performance - among all three types of assistance. This indicates that none of the tested navigation assistance affects scene memorability during exploration of a novel environment.

These results support findings from previous works on image memorability (Isola, Xiao, et al., 2011), which showed that people - in general - tend to remember the same images when presented with an image dataset and asked to remember presented images. 
Moreover, our findings are in agreement with a few studies on human navigation, which show that different navigation systems (Krüger, Aslan, \& Zimmer, 2004) or different modes of exploration (Gale et al., 1990; Richardson, Montello, \& Hegarty, 1999) do not influence the acquisition and formation of landmark knowledge. However, our results are in opposition to findings that people who use $2 \mathrm{D}$ map for navigation remember more scenes than people who use a hand-held computer (Münzer et al., 2006), although, in that study, participants were not asked to memorize the navigation path.

We suspect that differences in cognitive load, incurred by the different types of navigation assistance, probably contributed to the absence of an effect of navigation assistance. Previous investigation has shown that cognitive load was higher for 2D map than for wearable assistance, which in turn was higher than for human guide (Xu et al., 2014). Thus, if the 2D map is enhancing spatial memory due to required additional attention towards an environment (compared to WVN and HUG), this effect might have been cancelled out by a higher level of cognitive load (i.e., mental effort required to locate oneself within an environment). However, it is also possible that visual attention shifts, between 2D map and an environment, which were necessary for navigation might impeded memorization of encountered scenes.

Also, as people report different cognitive load for different types of assistance (Xu et al., 2014) our experiment shows that scene memorability is unaffected by the type of assistance and corresponding cognitive load. This may indicate dissociation of spatial working memory, reported as cognitive load, and visual memory.

Based on our results, and previous works on human navigation and image memorability, we conclude that none of the compared types of navigation assistance has notable effect on scene memorability during guided indoor navigation. 


\subsubsection{Navigation Information Make a Scene Highly Memorable}

The objective of our second experiment was to compare how information contained within a scene, and scene location, affect its memorability. A two-way within-subject ANOVA showed that location and information factor confound with each other. Therefore, we discuss results for information category with respect to scene locations.

Results show that scenes that provide navigation information are significantly more memorable than scenes that do not contain such information. The only exception occurred for corridor scenes. This might be caused by the fact that only one of the corridor scenes contains navigation information, and fourteen of them do not. Therefore, the proportion of compared groups might be inadequate to show the difference between them. In general, the results support our hypothesis. Scenes that provide navigation guidance are more memorable than scenes without such information.

Our findings confirm that people use navigation signs in wayfinding tasks (TorresMéndez \& Cervantes-Jacobo, 2012). High false positive (FP) scores for the "ambiguous info" category of unvisited-scenes images may indicate that the participants encoded and remembered the semantic information obtained during the navigation. When presented with the image of an unvisited scene that contains ambiguous information - semantic information from this image is similar to the semantic information of some scene encountered during navigation - the participants tend to wrongly classify this unvisited scene as a visited one. Therefore, this may imply that the participants remember what they have done - and its semantical aspect, and not visual details - after the task. Hence, if they are presented with some images that contain information which seems to fit into where they have been (e.g., floor level, similar door, etc.) and they have relatively little time to decide, they may reason 'I cannot remember having seen exactly this, but I should have seen this given where I moved through, so I better answer "Yes, I have seen this scene"'. 
However, these results might be caused by insufficient or confusing navigational labelling used in the building, which make it inappropriate to draw clear conclusions. This issue is worth further investigation.

Nevertheless, based on our results and previous works on the importance of navigation signs, we think that scenes that contain navigation information are more memorable than scenes without such information.

\subsubsection{Scenes at Some Locations Are More Memorable}

Our experiment also aimed to investigate if scene location can predict its memorability. As location and information factors interact with each other, we discuss results for location category with respect to the information contained within the scene.

Our hypothesis predicts that scenes located at the lift lobbies, and in front of the visited meeting rooms, should be more memorable than scenes located at the junctions, or along the corridors. The results partially confirm our prediction. In particular, lift lobbies and meeting room entrances scenes, which contain navigation information, are more memorable than corridors scenes. However, scenes of junctions are also significantly more memorable than scenes of corridors and room entrances. This result might be caused by the fact that our dataset contains only one scene which contains navigation information and is located at a junction. Therefore, other factors might cause that scene to be particularly memorable.

Our results do not show any significant difference between scenes, with no navigation information, at different locations. Therefore, we cannot conclude that the participants remembered some scenes more precisely due to their localization function. A higher memorability of some scenes might be caused by the fact that these scenes contained navigation information. High false positive scores of unvisited, lift lobbies, scenes which contain ambiguous information, might confirm that the participants encoded semantic information obtained from the encountered places. In order to define better localization 
function (i.e., the relation between a visual scene location and its memorability) future work will be needed.

\subsubsection{Notable Scenes Are Not Always Memorable}

While analysing the results, we have been surprised by memorability of some of the visitedscenes (see Figure 16). We expected that an art structure - certainly easily noticeable located at the ground floor lift lobby (see Figure 16 (a)) will be highly memorable. However, only four participants (20\%) correctly recognized it during the visual memory retention test. This is especially interesting when we compare this scene with some more memorable scenes (see Figure 16 (b) $-45 \%$ and Figure 16 (c) - 90\%). Although these scenes do not seem to be particularly interesting and do not contain crucial navigation information, they turn out to be more memorable than the art structure. This finding may echo the thesis that unusual, aesthetically pleasing, or interesting scenes are not always highly memorable (Isola, Parikh, et al., 2011). During a recognition task, people tend to guess ${ }^{6}$. Thus, they are biased by general scene knowledge (semantic memory), and therefore they are more likely to rationalize than to actually recall their surroundings (Brewer \& Treyens, 1981; Hollingworth \& Henderson, 1998; Nurmi, Salovaara, Bhattacharya, Pulkkinen, \& Kahl, 2011). This may

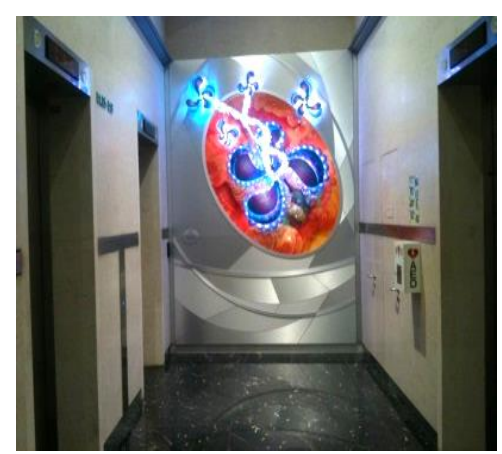

(a) mem. score $=\mathbf{2 0} \%$

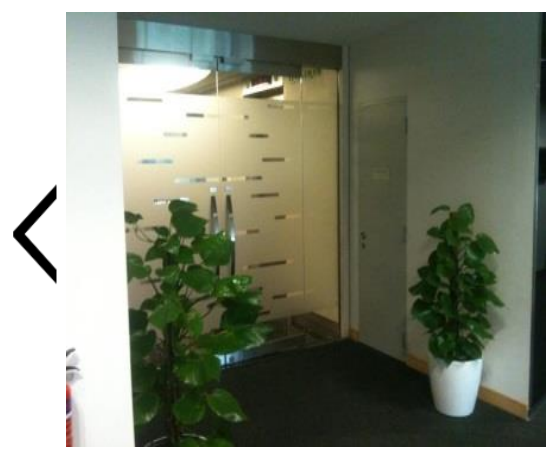

(b) mem. score $=45 \%$

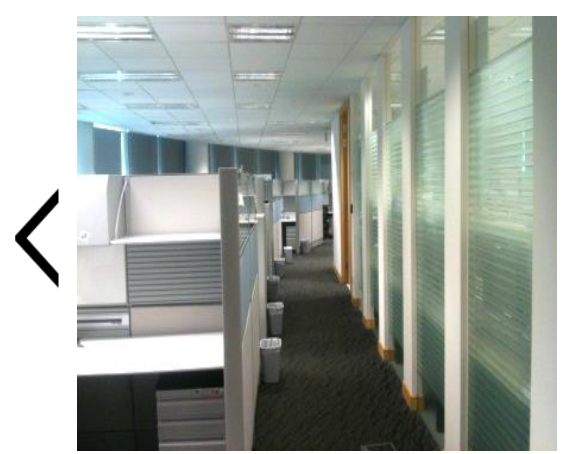

(c) mem. score $=90 \%$

Figure 16. Examples of visited scenes images with the memorability scores (mem. score). Memorability score indicates percent of the participants who correctly recognized particular scene. Sample size $n=20$. Adapted from Mukawa et al. (2017). 
partially explain why the art structure was not well memorized. Perhaps the participants unable to recognize this piece of art from their visual memory as it was probably not encoded into their long term visual memory, and being biased by their general knowledge encoded in their semantic memory - wrongly guessed that such a structure is rarely present at a lift lobby.

\subsubsection{Limitations and Future Research Directions}

Although our experiment provides valuable insights on scene memorability during guided navigation, we are fully aware of its limitations. We compared only three types of navigation assistance while a few more are available, e.g., mobile phone, a head-mounted display, or 3-D route planning (van Schaik, Mayouf, \& Aranyi, 2015). It might be of special interest to investigate how eyewear devices - equipped with head-mounted display - impact scene memorability, when used for guided navigation. Experiment described in the Chapter 4 (Do Navigation Assistance Interaction Modalities Affect Spatial Cognition) address this research issue.

Also, future studies should use a control group(s), which will navigate with a use of pre-installed indoor signage system, without any additional support. Moreover, due to time and resources constraints, we recruited 20 participants. Therefore, future studies may replicate our experiment on a larger number of participants to support our findings.

Indoor navigation assistance is especially important for people with cognitive impairment in navigation abilities, like: dementia, inability to localize oneself in an environment (Corkin, 1984), with difficulties in long-term planning (Luria, 1966), or with degraded cognitive processes (Rau \& Wang, 2003). It should be noted, that the participants recruited in this study are relatively young, and may not be representative of potential users (e.g., elderly, with mild cognitive impairment). Therefore, future studies should support our findings with a broader range of potential users. For example, the system could be evaluated in a situation of an elderly person who needs to find his or her way to a bank cashier in a new 
public building. Next, all the participants had normal or corrected-to-normal vision. Hence, it may be interesting to conduct the experiment with visually impaired people, to see how different types of navigation assistance, contained information, and location affects their memorability of encountered scenes. Finally, none of the participants has prior knowledge of the building (i.e., never visited the building before). Hence, it may be of special interest to compare scene memorability performance between first-time visitors and people who are familiar with an explored environment (i.e., visited it prior to an experiment).

Next, due to time constraints of the experiment, and the length of the navigation path, we studied the memorability of 40 scenes. Future studies may replicate our experiment on a longer, more complex route. This will allow defining and including a larger number of test scenes. It should be noted that we did not use specific criteria to choose our scenes. We tried to counterbalance visited-scenes dataset. Therefore, it consists of scenes that are equally spread among the navigation path and scenes that are most important for the navigation task. Also, in the future studies, the number of visited and unvisited scenes in particular information categories should be balanced; moreover, the number of scenes in particular location categories should be also balanced. This will allow to obtain stronger statistical results.

Moreover, in the future experiments, it would be of great benefit, to describe each image of a scene, with similarity and memorability score. These scores should be collected from independent judges (people that will not be aware of the aim of the main experiment). Therefore, future studies would have additional data to investigate if participants of guided navigation experiment remember the same scenes as judges; moreover, it will be possible to check how similarity of particular scenes affects their memorability scores, as well as false positive results. 
The confounding effect between information contained within a scene and its location made it inconclusive how those factors affect scene memorability. Future work is needed to better understand how navigation information influences scene memorability.

The images of the visited scenes were taken from the viewing perspective that imitates the participants' point of view. However, it is impossible to project all possible views, of all the participants, into one image. Therefore, some participants might perceive particular scenes from different perspectives than those presented as the test images. This could impact the recognition task. It might be useful to design future experiments with an emphasis on the selection of more realistic stimuli.

In addition, as all the participants in our study managed to successfully find their way back to the origin, it might be worth repeating the study with a more complex environment. This will help to understand which scenes people remember when faced with more complex navigation task. Will they still remember the same scenes, or will the task complexity affect their visual memory?

Also, our visual memory retention test was conducted around 5 minutes after a participant finished the navigation task. Due to time and resource constraints, we were not able to perform follow-up memory tests, to check the participants' performance after weeks (e.g., 2 weeks) and/or months (e.g., 1 and 3 months) of the experiment. In future, it may be interesting to check if the differences in participants' performance, or lack of them, are still present after weeks and/or months of the guided navigation task.

It is also worth investigating how top-down, task-specific attention affects scene memorability. We are especially interested in which scenes participants will memorize when they are not asked to remember the navigation path, but instead we asked them just to follow the experimenter. They will be instructed that a test will be carried out in the meeting room, and they will not be aware that the test checks what they remembered on the way. Will 
participants remember the same scenes, as in the case when they were asked to remember the path, or will they remember some other places?

\subsection{Repetition Priming and Visual Memory - Introduction and Methodology}

Our vision is essential for efficient exploration of a novel environment. However, we cannot process all perceived visual input; it is attention that allows us to optimize performance in a visual task. Attention affects which parts of a visual scene we will process; it impacts memories. Our attention is driven not only by currently perceived stimuli (bottom-up attention), but also by our memories (top-down attention).

Priming affects our top-down attention, which is driven not only by explicit, by also by implicit memories. What we have recently seen and focus our attention to, strongly influence how we will allocate our future attention (Maljkovic \& Nakayama, 1994). Therefore, attention affects which scenes will or will not be processed; it indirectly affects visual memory of that scene.

Studies on repetition priming investigate effects of prior presentation of a stimulus on one's response to the same stimulus when it is presented at a later time. These priming pattern results from matching currently perceived stimuli with a memory representation of the previous trial (L. Huang, Holcombe, \& Pashler, 2004). In addition, priming effects are visible even in people who do not remember the priming procedure; their implicit memory is affected (Warrington \& Weiskrantz, 1974).

As some visual scenes are more memorable than others, independent of the perceivers' memories of other biases (Isola, Xiao, et al., 2011), our study aims to investigate how repetition priming affects our visual memory during backtracking in a novel environment. To the best of our knowledge, our experiment is the first work which investigates this question. Moreover, we check how backtracking alone improves memorization of encountered places. 
Ultimately, understanding the effect of backtracking and repetition priming on visual memory during wayfinding in a novel environment may allow us to design better tools and training procedures in support for this demanding task.

Our experiment investigates if repetition priming improves visual memory of visited places during backtracking in a novel environment. It also checks if backtracking alone improves memory of these places. The participants were asked to remember the taken path, as they would need to backtrack by themselves. A visual memory retention test was carried out at different locations for two separate groups of the participants. The test checked which visited scenes were remembered after guided wayfinding and after backtracking

\subsubsection{Participants}

20 adults (11 males and 9 females) were recruited. Ages ranged between 21 and 36 years old (mean age 26.2 years with standard deviation of 4 years). All participants had normal or corrected-to-normal vision. The participants had never visited the experimental environment (i.e., the institute building) before and had no prior knowledge of the office layout. The participants were randomly split into two groups: $1^{\text {st }}$ Group: 6 males and 4 females; $2^{\text {nd }}$ Group: 5 males and 5 females. The $1^{\text {st }}$ Group underwent the visual memory test twice: at the destination location, and after backtracking to the starting location. The $2^{\text {nd }}$ Group underwent the test only once: after backtracking to the starting location.

\subsubsection{Apparatus}

Similarly to our first experiment, the visual memory retention test was carried out with the use of a tablet PC. For each shown image (i.e., stimulus), a participant needs to decide (by pressing either 'Yes' or 'No' button) if he or she remember the presented scene. Each image is displayed for a maximum of 5 seconds. The distance between a participant's eyes and the tablet screen varied: between 30 and $60 \mathrm{~cm}$. For these distances, participants had seen the images at $45 \times 18$, and $24 \times 9$ degrees of visual angles respectively. 


\subsubsection{Stimuli}

80 images of indoor scenes were collected. Half of them were taken along the wayfinding path. These images are denoted as visited scenes. We chose these scenes so that they are spread equally along the navigation path. Opposite to the previous study, in this one, we did not categorize images into distinct groups - neither based on information they contain (i.e., "no info" or "navigation info"), nor on their location (i.e., junction, lift lobby, corridor, or meeting room entrance). The other half were taken at different parts of the office environment, but at locations unseen from the wayfinding path. This subset is denoted as the unvisited scenes. Images of both visited and unvisited scenes, were mixed together and presented to a participant in a random order

\subsubsection{Procedure}

The experiment was carried out for individual subjects. After informed consent was obtained, a participant was led to the starting point. A participant was instructed to remember the taken path, as he or she will need to backtrack without any support. Next, the participants were guided to the destination location. The visual memory retention test, for the $1^{\text {st }}$ Group, was carried out at the destination location. Before the test, a participant was asked to fill up a few questionnaires that were not related to the study. It took around $3-5$ minutes; therefore, we assume that the visual memory retention test examines the participant's long-term visual memory.

During the visual memory retention test, 80 images of scenes were shown to the participants. For each presented scene, the participant needs to decide if he or she had seen the scene along the travelled path. The participants did not receive feedback if their answer is correct or not. Based on the data collected from the test, we got information about which scenes were remembered by a particular participant. After completing the visual memory retention test, a participant was asked to backtrack to the starting location. During 
backtracking, no assistance was provided. At the starting location, all participants $\left(1^{\text {st }}\right.$ and $2^{\text {nd }}$ Group) were asked to fill up a few questionnaires, and to conduct the visual memory test.

\subsubsection{Hypotheses}

Our first hypothesis states that, the participants should correctly recognize more encountered scenes after backtracking to the starting location, than after guided wayfinding (i.e., at the destination location). This hypothesis is based on current findings on human wayfinding. People use landmarks as decision points when choosing a correct route (Foo et al., 2007). Therefore, during backtracking their attention should be attracted by those scenes that contain valuable wayfinding information. Visual memory of those scenes will be enhanced, and the recognition performance, during the memory test, should be higher.

Our second hypothesis predicts that, the repetition priming - memory test performed at the destination location, that presents mixed visited and unvisited places in random order will further improve visual memory during backtracking. Our attention is driven not only by currently perceived stimuli, or given task, by also by our explicit and implicit memories; therefore, participants exposed to the test images - priming - should consciously or unconsciously look for the presented scenes during backtracking. Moreover, the participants may be curious how well they memorized visited scenes, as they became aware that we test their visual memory. Some participants may also expect that they will be tested again, after backtracking to the starting location. In general, all the participants exposed to the priming should memorize more scenes after backtracking, than the participants who do not conduct the memory test at the destination location.

\subsection{Repetition Priming and Visual Memory - Results}

Here we present results collected from twenty participants (10 in the $1^{\text {st }}$ Group and 10 in the $2^{\text {nd }}$ Group). All participants successfully found the destination location during guided wayfinding. Also, all participants were able to backtrack by themselves to the starting 
location. Based on the data collected during the visual memory test, we calculated a visual memory score for each participant. The memory score is defined as the percentage of correct recognitions (i.e., true positive) of the visited scenes.

\subsubsection{The effect of backtracking on visual memory}

To check how backtracking task enhance visual memory of visited scenes, we compared the performance of the $1^{\text {st }}$ Group at the destination location with the performance of the $2^{\text {nd }}$ Group after backtracking (see Figure 17 ). The average memory score of the $1^{\text {st }}$ Group at the destination location was $49.8 \%(S E=2.5 \%)$, and the average memory score of the $2^{\text {nd }}$ Group (i.e., only one test, after backtracking) was $62 \%(S E=4.6 \%)$. Independent-measures t-test shows significant improvement $[t(18)=2.13, p=0.047]$ of visual memory after backtracking. Moreover, high correlation $\left(r_{s}=0.71\right)$ between the scenes memorized by the 1 st and 2 nd

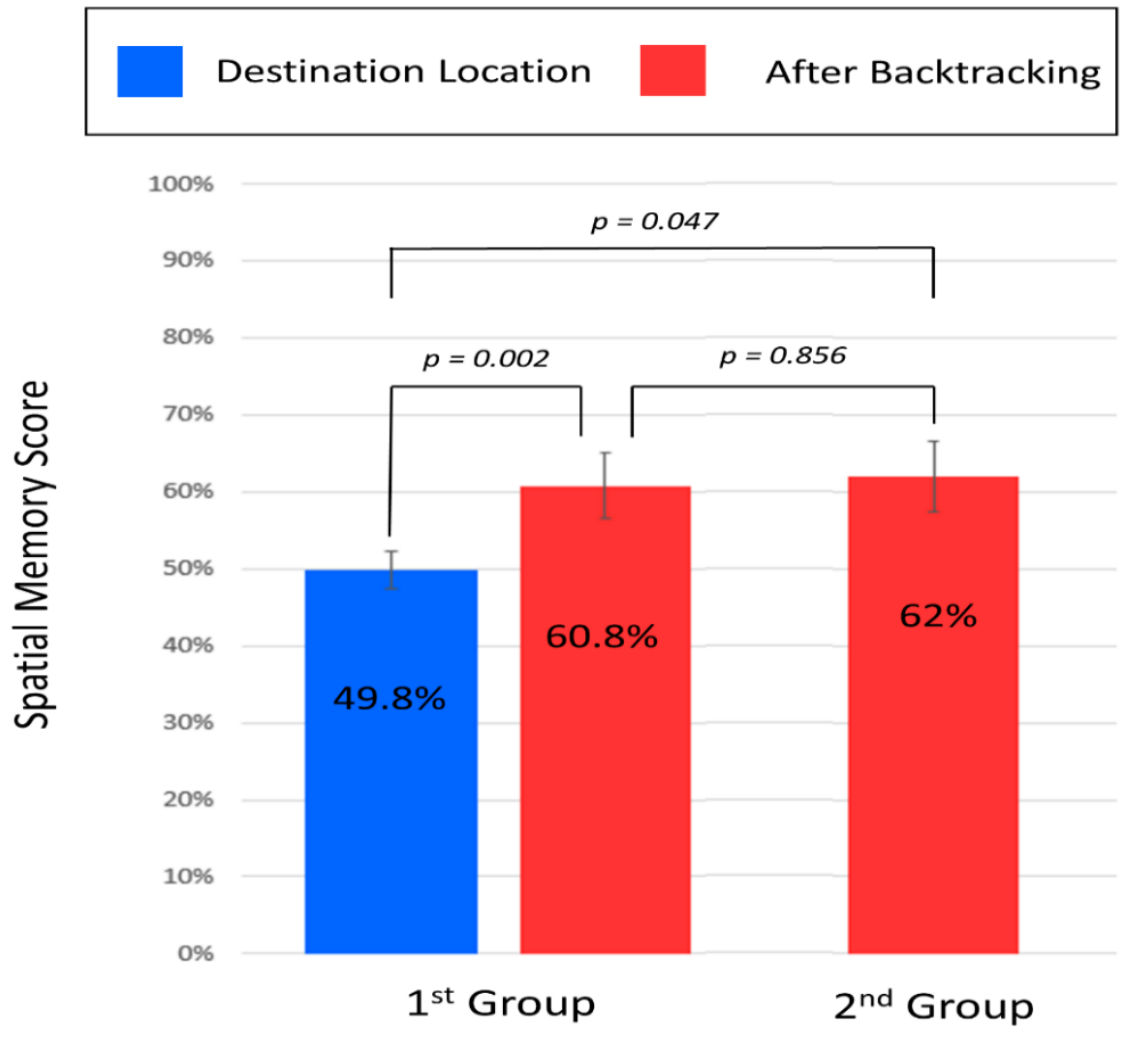

Figure 17. Average visual memory scores for the $1^{\text {st }}$ Group (at destination location and after backtracking) and for the $2^{\text {nd }}$ Group (only after backtracking) of the participants. Error bars show the standard error. Sample size $n=20$. Adapted from Mukawa et al. (2015). 
Group, indicates that the participants memorized the same scenes during guided wayfinding and after backtracking.

\subsubsection{The effect of repetition priming on visual memory}

$1^{\text {st }}$ Group of the participants was exposed to repetition priming - the visual memory retention test at the destination location. After backtracking the same group was asked to repeat the memory test. This procedure was used to investigate if - and if yes, how strongly - repetition priming affects further enhancement of visual memory during backtracking. Matched-pair (dependent-measures) t-test shows that the memory score of the $1^{\text {st }}$ Group improved significantly $[t(9)=-4.3, p=0.002]$ from $49.8 \%$, at destination location, to $60.8 \%$, after backtracking (see Figure 17). Surprisingly, no significant difference, with use of independentmeasures t-test, $[t(18)=0.18, p=0.856]$ was found in memory performance between the $1^{\text {st }}$ Group (i.e., exposed to repetition priming) and the $2^{\text {nd }}$ Group (i.e., tested only after backtracking). These results indicate that repetition priming does not enhance memorization of encountered locations. Finally, high correlations, between scenes memorized by different groups, show that repetition priming does not affect selection of memorized scenes (see Figure 18).

\subsection{Repetition Priming and Visual Memory - Discussion}

Our experiment explores how backtracking and repetition priming affect visual memory during wayfinding in a novel environment. Our hypotheses assumed that: (1) people should remember more visited scenes after backtracking to the starting location, compared to after guided wayfinding to the destination location; (2) repetition priming, in form of visual memory test at destination location, should result in further enhancement of the visual memory during backtracking.

The results confirm our first prediction. After backtracking, the participants correctly recognized more encountered scenes. Their attention is guided by a given task - backtrack to 
the starting location. They enhance visual memory of encountered landmarks, which provide wayfinding information. Hence, their visual memory of encountered scenes is strengthened. Moreover, during the search for landmarks, the participants probably memorize other, less significant but memorable, objects or visual scenes.

On the other hand, our second hypothesis is not supported by the results. Repetition priming does not further enhance memorization of these scenes. The participants exposed to priming memorized similar number of scenes as the participants who were not exposed to priming. This may be explained by the following reasons. Firstly, our attention is strongly guided by a given task, and may be independently of priming (Leonard \& Egeth, 2008). Therefore, it is possible that the participants focused only on the backtracking task and were unreceptive to the priming effect. Secondly, the cognitive load-associated with the backtracking task - might be too high, and thwarts further, conscious or unconscious, improvement of visual memory.

\section{Groups Correlations}

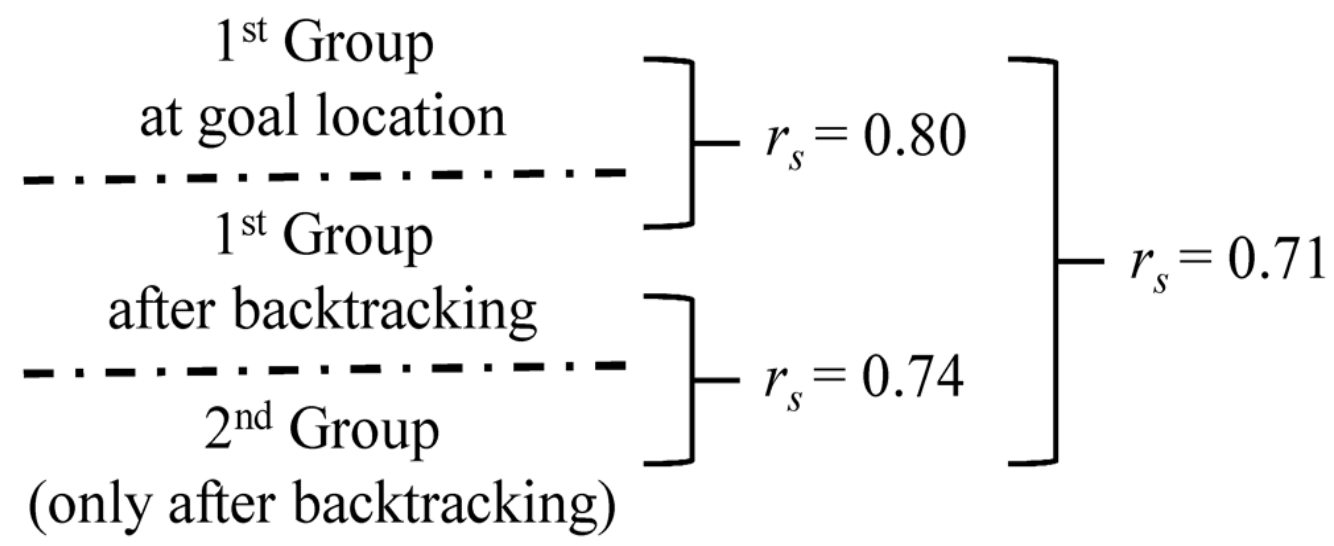

Figure 18. Correlations between scenes memorized by the different groups of participants. Adapted from Mukawa, Tan, Lim, $\mathrm{Xu}$, and $\mathrm{Li}$ (2015). 
High correlations between scenes memorized by different groups show that most of the participants remember the same visited places. This finding echoes the previous works on image memorability, which showed that people tend to remember the same images (Isola, Xiao, et al., 2011).

In the future, a modification of our experiment may bring some additional findings. We would like to investigate how backtracking and repetition priming affects people's visual memory when they are not deliberately asked to remember a navigation path.

In general, our findings provide valuable insights on how backtracking and repetition priming affects visual memory during wayfinding in a novel environment. These findings can be used to design better tools and training procedures to support this demanding task. 


\section{Do Navigation Assistance Interaction Modalities Affect Spatial}

\section{Cognition?}

\subsection{Introduction and Methodology}

This experiment investigates if Google Glass (GG) is able to provide navigation assistance as efficiently as a person familiar with an environment - Human Guide (HG). Also, it compares the efficiency of different GG interaction modalities (i.e., voice, display, voice+display). We check which of the modality is the more effective and acceptable by a user. Finally, we investigate how different types of navigation assistance, and different interaction modalities, affect users' spatial cognition - navigation and backtracking performance, correct backtracking decision, and visual memory. In addition, we evaluate participants' subjective experience (i.e., enjoyment, ease-of-use, etc.) of using a particular navigation tool. Focusing on how GG affects user's spatial cognition, and through usability evaluation, results of this experiment are applicable to scenarios such as navigation in hospitals, schools, shopping malls, airports, etc.

We used Google Glass to implement wearable navigation assistance. Hence, we compare two types of navigation assistance, Google Glass (GG) and Human Guide (HG). For each participant, GG provided navigation instructions in one of three interaction modalities: voice, display, or voice+display.

In general, our main research questions for this study include:

(1) Is user's spatial cognition affected by the use of GG, with different interaction modalities, when compared to being guided by the HG?

(2) Which type of navigation assistance is most effective? In particular, is GG able to provide navigation assistance as efficiently as a person (HG) familiar with an environment? 
(3) Which GG interaction modality is the most beneficial for the users?

This experiment evaluated the participants' navigation and backtracking performance under different conditions. Furthermore, we checked if (and how) the participants' visual memory is affected. Two independent variables were examined. The first was the type of navigation assistance, i.e., GG vs. HG. The second independent variable was the interaction modality in which GG provided navigation information. Three different modalities were examined: voice vs. display vs. voice+display. These modalities are described in more detail in the following section (Apparatus).

Table 4 shows our experiment design. We used within-subject (2 types of navigation assistance) and between-subject (3 interaction modalities) design. Each participant was guided along two routes, in each of them, he or she used different navigation assistance (GG or $\mathrm{HG})$. The order of assistance usage was random and counter-balanced. When GG was used for navigation, the following conditions were specified for different groups of participants: C1: voice modality; C2: display modality; C3: voice+display modality. The order of conditions was random and counter-balanced.

Table 4. Google Glass for Indoor Navigation - Experiment design.

\begin{tabular}{|c|c|c|c|c|}
\hline & Condition 1 (C1) & Condition 2 (C2) & Condition 3 (C3) \\
\hline \multicolumn{2}{|c|}{$\begin{array}{c}\text { Google Glass Interaction } \\
\text { Modality }\end{array}$} & voice & display & voice+display \\
\hline \multirow{2}{*}{$\begin{array}{l}\text { Navigation } \\
\text { Assistance }\end{array}$} & $1^{\text {st }}$ Route $\left(R_{1}\right)$ & Google Glass & \multicolumn{2}{|c|}{ Human Guide } \\
\hline & $2^{\text {nd }}$ Route $\left(\mathbf{R}_{2}\right)$ & Human Guide & iide & oogle Glass \\
\hline
\end{tabular}




\subsubsection{Participants}

Our results cover data collected from 51 participants (26 males and 25 females), with ages range between 19 and 39 years old (Mean = 23.9 with the standard deviation of 3.6 years). Each group has 17 participants. All participants had normal or corrected-to-normal vision. Each assisted navigation session lasted for about 50 minutes. The participants have no prior knowledge of the building where the experiment was conducted. The participants visited three different office levels. The environment consists of a typical office space with cubicles, offices, meeting rooms, pantries, and lift lobbies.

\subsubsection{Apparatus}

The participants used two different types of navigation assistance: GG (see Figure 19), and HG. When HG assistance is used, the experimenter provides verbal directions necessary for reaching a destination, like: "Please go straight ahead", "Please turn right", etc. When GG assistance is used, it provides location-based assistance to guide a participant. We prepared a mock-up application installed on Google Glass and controlled by an experimenter from a mobile phone. However, the participants were informed that the guidance is provided by an automated navigation system, which recognizes the environment (with the use of GG camera) and provides corresponding navigation instructions. Actually, the experimenter, while following a participant, was activating predefined navigation information at particular route locations. GG provides navigation information in one of the three modalities: (1) voice commands with the use of GG speaker, (2) show instruction on the Glass display, (3) combination of both, voice+display information. In all three modes, a navigation instruction is preceded by a prompt sound (i.e., Android system Success Sound), which informs a participant about a new navigation instruction. On the display, a participant may see: an arrow that indicates navigation direction, a lift icon with additional information about the level to which he or she should take a lift, an icon that indicates that a participant reached a 


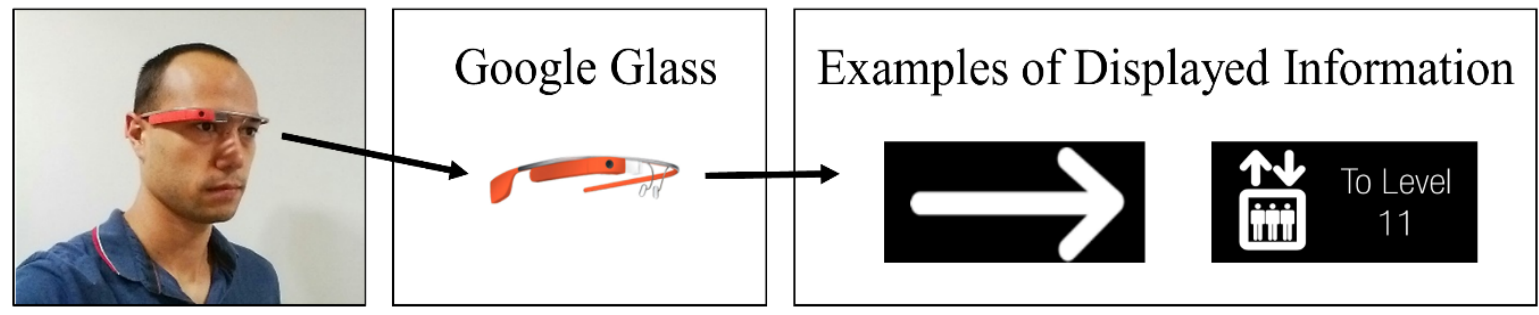

a)

b)

c)

Figure 19. Navigation assistance with use of Google Glass, a) a person wearing the Google Glass, b) the Google Glass, c) samples of navigation information displayed on the Google Glass display.

final location (see Figure 19 (c)). The navigation instruction is displayed for 3 seconds - time was specified based on our preliminary user study. When voice commands are specified as the modality, a participant hears a navigation instruction; for example: "Please go straight ahead", "Please turn right", etc. When GG operates in the third modality, a participant sees an instruction and also hears a corresponding voice command.

To check how a particular navigation assistance, or interaction modality, affects participants' visual memory, we designed a visual memory retention test. The test was carried out using a desktop computer with a 17', 4:3 aspect ratio monitor. The participants performed the test while sitting on a typical office chair in front of a monitor. The distance between a participant's eyes and the monitor screen is between 70 and $100 \mathrm{~cm}$. At these distances, participants viewed the images at $28 \times 15$, and $19 \times 11$ degrees of visual angles, respectively. The test was conducted with a simple applet. For each presented image there are two possible answers: 'Yes' - if a participant thinks that he or she visited the place presented on the image; and 'No' - if the participant thinks otherwise. An answer is chosen by pressing either "Yes" or "No" button - green or red labels with text, glued to a computer keyboard keys. Each image is presented for a maximum of 5 seconds. We have chosen 5 seconds period based on our preliminary user study. The time remained for an answer is displayed in 
a form of a decreasing progress bar. When no answer is provided within 5 seconds, the system saves 'No Response' information.

For the visual memory retention test, we collected sixty images of indoor scenes. Half of them were taken along the navigation path. Therefore, this subset of images is denoted as the visited-scenes. We chose these places so that the dataset consists of scenes that are equally spread along the navigation path. The other half of images were taken in different parts of the same indoor environment, but at locations outside line-of-sight from any point along the navigation path. This subset of images is denoted as the unvisited-scenes. These places are selected so that the number of scenes at particular locations is balanced. During the test, images of both visited- and unvisited-scenes were mixed together and presented to a participant in a random order.

\subsubsection{Independent Variables}

\subsubsection{Navigation Assistance}

Two types of navigation assistance were compared: GG and HG. Both types of assistance provide just-in-time information and require less effort from the participants than other types of navigation aids (e.g., 2D map, digital map, etc.).

When GG uses voice as the interaction modality it is most similar to how HG provides guidance. With the use of only voice information, a user's visual attention should not be affected. However, when using GG display users may still need to switch their visual attention between the display and the environment. Therefore, our experiment investigates if users' visual attention, and indirectly their spatial cognition, is affected by the type of assistance used for navigation and by the interaction modality of the GG.

HG assistance is probably the most reliable navigation aid. A guide has an expert knowledge of an environment and provides just-in-time information. Moreover, a guide can immediately react to a traveller's incorrect action by showing a correct direction. Therefore, 
in our experiment we used $\mathrm{HG}$ as the optimal means of navigation assistance, so as to compare with GG-based wearable navigation assistance.

\subsubsection{Interaction Modality}

While guided by HG assistance, the participants were informed which direction to go in order to reach a destination. Navigation information was provided at locations with more than one possibility of further movement.

While guided by GG assistance, three modalities can be used to present the navigation information, namely, voice, display, and voice+display. Navigation information provided with the use of any of the three modalities was orientation-sensitive and gives timely information about which direction to take. Similarly to $\mathrm{HG}$ assistance, information was provided at location with more than one possibility of further movement.

\subsubsection{Dependent Variables}

For dependent variables, we assessed two categories of variables: objective measures, and subjective measures.

Objective measures include:

- $\quad$ Time needed to complete a particular route (NavTime),

- $\quad$ Time needed to backtrack on a particular route (BackTime),

- $\quad$ Correct backtracking decisions (CorrectDecisions),

- $\quad$ Visual memory score of scenes encountered along a route (MemoryScore).

For NavTime and BackTime, we normalized the time for each route among all experiment conditions. The normalization was intended to cancel out the effect of varied locomotion speed of each participant, route length, and other conditions. From all the collected navigation times, we computed the mean time $(\mu)$ and standard deviation $(\sigma)$. The normalized time $t_{i}^{n}$ for a participant $i$ was computed as: $t_{i}^{n}=\left(t_{i}-\mu\right) / \sigma$. The backtracking time (BackTime) was normalized using the same formula. 
During backtracking, each participant had to make a navigation decision at the route junctions. The correct backtracking decisions (CorrectDecisions) for each participant was calculated as: (number of correct navigation decisions during backtracking) / (sum of necessary navigation decisions during backtracking).

Based on the data collected from the visual memory retention test, we obtained the MemoryScore for each participant. The MemoryScore is defined as the percentage of correctly recognized images of scenes encountered along a route.

Subjective measures address user experience. The participants were asked to fill up two questionnaires - one for each navigation assistance. The questionnaires used a seven-point Likert Scale. Seven measures (listed below) were evaluated, each consisting of 3-4 items.

- Usefulness - how useful the navigation assistance is during the task,

- Ease-of-use - how easy it is to use the navigation assistance during the task,

- Cognitive load - how much mental effort the user has to make to complete the task,

- Enjoyment - how enjoyable it is to use the navigation assistance,

- $\quad$ Stress level - how stressful the user feels during the task,

- Intelligence - to what extent the user perceives the navigation assistance as intelligent,

- $\quad$ Trust - how trustful the information provided by the navigation assistance are.

\subsubsection{Procedure}

A general procedure of the experiment is as follows. First, a participant was led to the starting point $\left(\mathrm{P}_{0}\right)$, where he or she was informed about the experiment structure and procedure. At this stage, informed consent was obtained. Next, we presented GG and explained how it works. Sample navigation information (in the form of one of the modalities) was presented to the participant to ensure he or she is familiar with the system. If needed, Glass display was 
adjusted to the participant eyesight. As most of the participants had not used GG before, we have given them enough time to get familiar with the system until they felt confident to start the experiment.

All the participants were guided along two routes. They were asked to follow the navigation assistance instructions in order to reach their final destination for each route. The first route $\left(\mathrm{R}_{1}\right)$ leads from starting point $\left(\mathrm{P}_{0}\right)$ to a meeting room $\left(\mathrm{P}_{1}\right)$. This route consists of 5 junctions and covers two building levels, a lift is used to move between the levels. The second route $\left(\mathrm{R}_{2}\right)$ leads from $\mathrm{P}_{1}$ to another meeting room $\left(\mathrm{P}_{2}\right)$. This route consists of 6 junctions and also covers two levels (also, a lift is used). The participants used different navigation assistance (i.e., GG or $\mathrm{HG}$ ) in each route. A participant used each navigation assistance only once. The sequence of routes was fixed for each participant for experimental control reasons. Sequence effect was eliminated by balancing the order of the navigation aids. Some participants were guided along the $\mathrm{R}_{1}$ route by $\mathrm{HG}$ and along the $\mathrm{R}_{2}$ route by $\mathrm{GG}$, while others were guided along the $\mathrm{R}_{1}$ route by $\mathrm{GG}$ and along the $\mathrm{R}_{2}$ route by $\mathrm{HG}$.

The time needed to travel along a particular route was recorded to compute the NavTime. At the last destination (i.e., $\mathrm{P}_{2}$ ), a participant was asked to fill up the two user experience questionnaires. This took around 5-10 minutes; therefore, we assume that the memory test - performed next - examine a participant's long-term visual memory (Atkinson \& Shiffrin, 1968). During the test, 60 images of scenes - in a random order - were shown to a participant. For each presented scene, a participant needs to decide (by pressing either 'Yes' or 'No' button) if he or she encountered the presented scene along the route. This data allowed us to compute MemoryScore for each participant.

After completing the visual memory retention test, a participant was asked to backtrack along the two navigation routes. First, a participant was asked to find the way to the first meeting room (i.e., $\mathrm{P}_{1}$ ). Next, he or she was asked to find the way to the starting 
location (i.e., $\mathrm{P}_{0}$ ). During backtracking, no assistance was provided. If a participant made a mistake (e.g., took a wrong turn at a junction), he or she was corrected. Times needed for backtracking to each of the locations were recorded and used to compute the BackTime for each of the routes. Also, the number of correct backtracking decisions was counted and used to compute the CorrectDecisions.

\subsubsection{Hypotheses}

We investigate how different types of navigation assistance and interaction modalities affect users' spatial cognition (i.e., navigation and backtracking), as well as the visual memory of scenes encountered along the route.

We expect that GG assistance, with voice interaction modality, emulates a HG to achieve equivalent performance with respect to navigation and backtracking time (i.e., NavTime and BackTime). This is based on the fact that both types of assistance use only voice to provide navigation information. Moreover, we expect no differences in the number of correct backtracking decisions, and visual memory scores between the participants who used GG with the voice modality, and the participants guided by HG.

For the display, and voice+display interaction modalities of GG, we expect that it will take participants longer to navigate when compared to use of HG. Moreover, we also expect that backtracking performance, number of correct backtracking decisions, and visual memory will be worse for participants who use GG with the display and voice+display modalities when compared to HG users. We think that GG users need to shift their visual attention between the Glass display and the environment. While doing it, a participant may not assimilate enough environment information - like a floor layout or visual information needed for efficient navigation, backtracking, and remembering encountered scenes. However, during data analysis we may expect some deviations. They may happen if a particular participant(s), while guided by GG with voice+display modality, will be using only 
voice information, while not shifting his or her visual attention to the Glass display. However, even in such case, it is high probably that participant's visual attention will be involuntary shifted towards the Glass display. In general, performance differences, between HG and GG with voice and voice+display modalities, will allow us to analyse how users' spatial cognition is affected.

The hypotheses in the study are presented in Table 5. The comparison is made with respect to the values of individual measurements. The " $\mathrm{X}=\mathrm{Y}$ " formula means that there is no statistical significance difference between compared conditions. The " $\mathrm{X}<\mathrm{Y}$ " formula means that the obtained result is significantly higher for condition $\mathrm{Y}$ than for condition $\mathrm{X}$.

Table 5. Hypotheses for different interaction modalities (GG - Google Glass, HG - Human Guide).

\begin{tabular}{|c|c|c|}
\hline Dependent Variable & & Hypotheses \\
\hline $\begin{array}{l}\text { Navigation Time } \\
\quad \text { (NavTime) }\end{array}$ & $\mathrm{H} 1$ & $\begin{array}{c}\mathrm{GG}_{\text {Voice }}=\mathrm{HG} \\
\mathrm{GG}_{\text {Display }}=\mathrm{GG}_{\text {Voice+Display }}>\mathrm{HG}\end{array}$ \\
\hline $\begin{array}{l}\text { Backtracking Time } \\
\quad(\text { BackTime })\end{array}$ & $\mathrm{H} 2$ & $\begin{array}{c}\mathrm{GG}_{\text {Voice }}=\mathrm{HG} \\
\mathrm{GG}_{\text {Display }}=\mathrm{GG}_{\text {Voice+Display }}>\mathrm{HG}\end{array}$ \\
\hline $\begin{array}{l}\text { Correct Backtracking } \\
\text { Decisions } \\
\text { (CorrectDecisions) }\end{array}$ & H3 & $\begin{array}{c}\mathrm{GG}_{\text {Voice }}=\mathrm{HG} \\
\mathrm{GG}_{\text {Display }}=\mathrm{GG}_{\text {Voice+Display }}<\mathrm{HG}\end{array}$ \\
\hline $\begin{array}{l}\text { Visual Memory } \\
\text { (MemoryScore) }\end{array}$ & $\mathrm{H} 4$ & $\begin{array}{c}\mathrm{GG}_{\text {Voice }}=\mathrm{HG} \\
\mathrm{GG}_{\text {Display }}=\mathrm{GG}_{\text {Voice+Display }}<\mathrm{HG}\end{array}$ \\
\hline
\end{tabular}

\subsection{Results}

In this section, we present results collected from fifty-one participants. We show how different types of navigation assistance and interaction modalities affect users' spatial cognition - in performing navigation and backtracking. Next, we present how these factors influence users' visual memory. Finally, we analyse subjective user experience. The 
navigation and backtracking times were normalized. After analysis of the visual memory retention test data, we noticed that the number of "No response" answers accounted for approximately $0.5 \%$ of all the answers. Therefore, for simplicity, "No response" was considered as a "No" answer during data analysis.

For data analysis, we used one-way ANOVA with independent measures, as well as Kruskal-Wallis test; for post-hoc analysis we used t-tests, Wilcoxon and Mann-Whitney tests for non-parametric data. The significance level was set at $5 \%$.

\subsubsection{Navigation and Backtracking Performance}

Figure 20 shows navigation and backtracking performance under different conditions. The navigation and backtracking times obtained with the use of GG operating in different interaction modality (i.e., voice, or display, or voice+display) are also obtained with the use of HG. Table 6 shows navigation time differences between different types of navigation assistance. During navigation, the participants who used GG with the voice modality, needed more time to reach the goal location than the participants who used HG. Similarly, when the participants used GG with the display modality, it took them longer to complete the task. However, when using GG with the combination of both modalities - voice+display, the participants were able to complete the task as efficiently as the group guided by HG. 

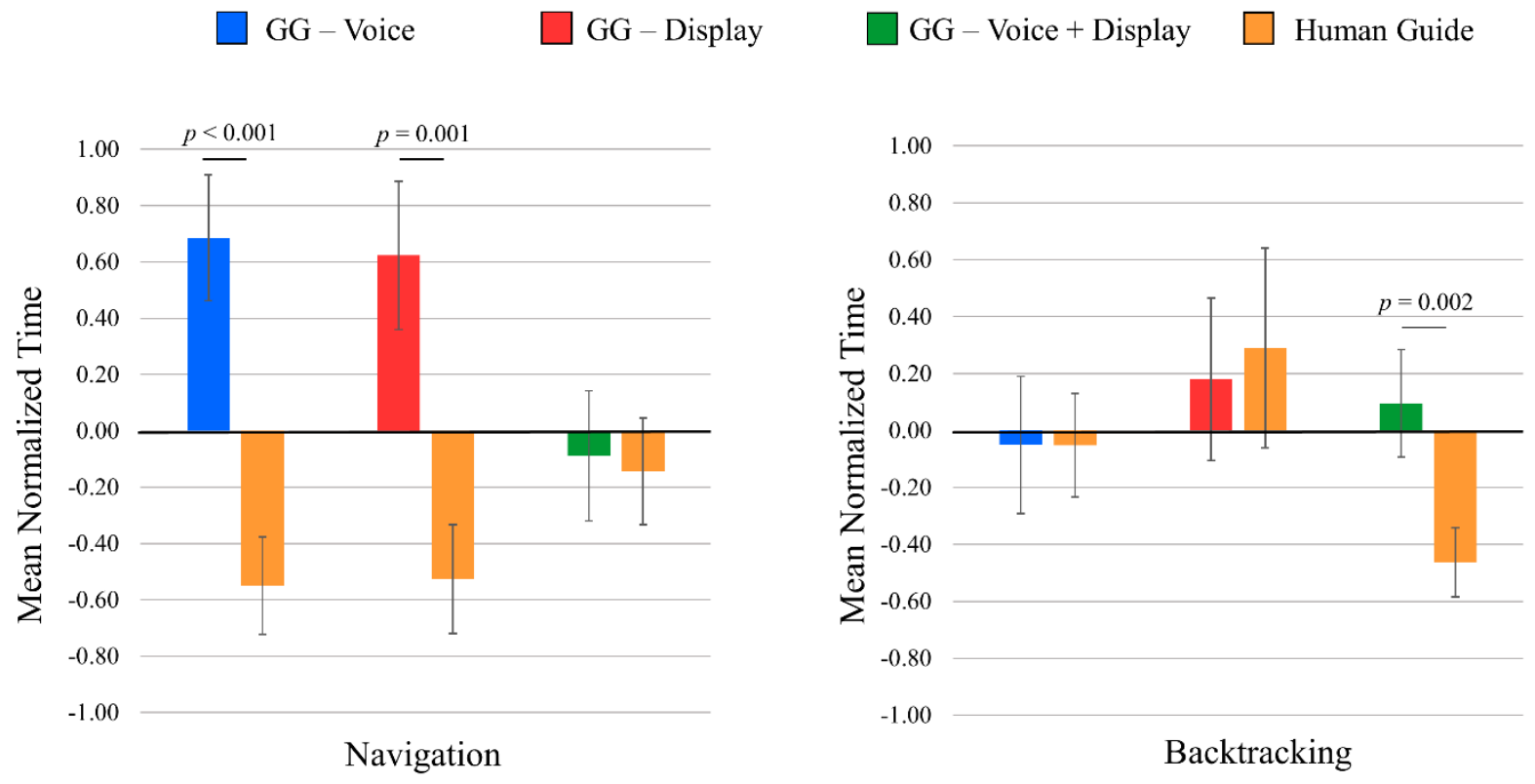

Figure 20. Mean navigation and backtracking times with the use of different types of assistance and different Google Glass modalities. Error bars show standard error. Sample size $n=51$ (17 participants in each group - GG-Voice, GG-Display, GG-Voice+Display).

Table 6. Navigation Time T-test Results Between Different Types of Navigation Assistance.

\begin{tabular}{|c|c|c|c|}
\hline & \multicolumn{3}{|c|}{ Google Glass Interaction Modality } \\
\hline Navigation Assistance & voice & display & voice+display \\
\hline Google Glass Mean $_{\text {NavTime }}$ & 0.69 & 0.62 & -0.09 \\
\hline Human Guide Mean $_{\text {NavTime }}$ & -0.55 & -0.53 & -0.14 \\
\hline T-Test & $\begin{array}{c}t(16)=5.22 \\
p<0.001\end{array}$ & $\begin{array}{c}t(16)=3.93 \\
p=0.001\end{array}$ & $\begin{array}{c}t(16)=0.32 \\
p=0.75\end{array}$ \\
\hline
\end{tabular}

Table 7 shows navigation time ANOVA and t-test results for different interaction modalities of Google Glass. The between subjects one-way ANOVA showed significant differences between navigation times acquired with the use of GG with respect to different 
modalities. We found no difference between navigation performance with the use of GG in the voice or the display modality. However, there were significant differences between remaining modalities: voice vs. voice+display, and display vs. voice+display. For the navigation times acquired with $\mathrm{HG}$, one-way ANOVA showed no significant differences between different groups.

Table 7. Navigation Time ANOVA and T-test Results for Different Interaction Modalities of Google Glass.

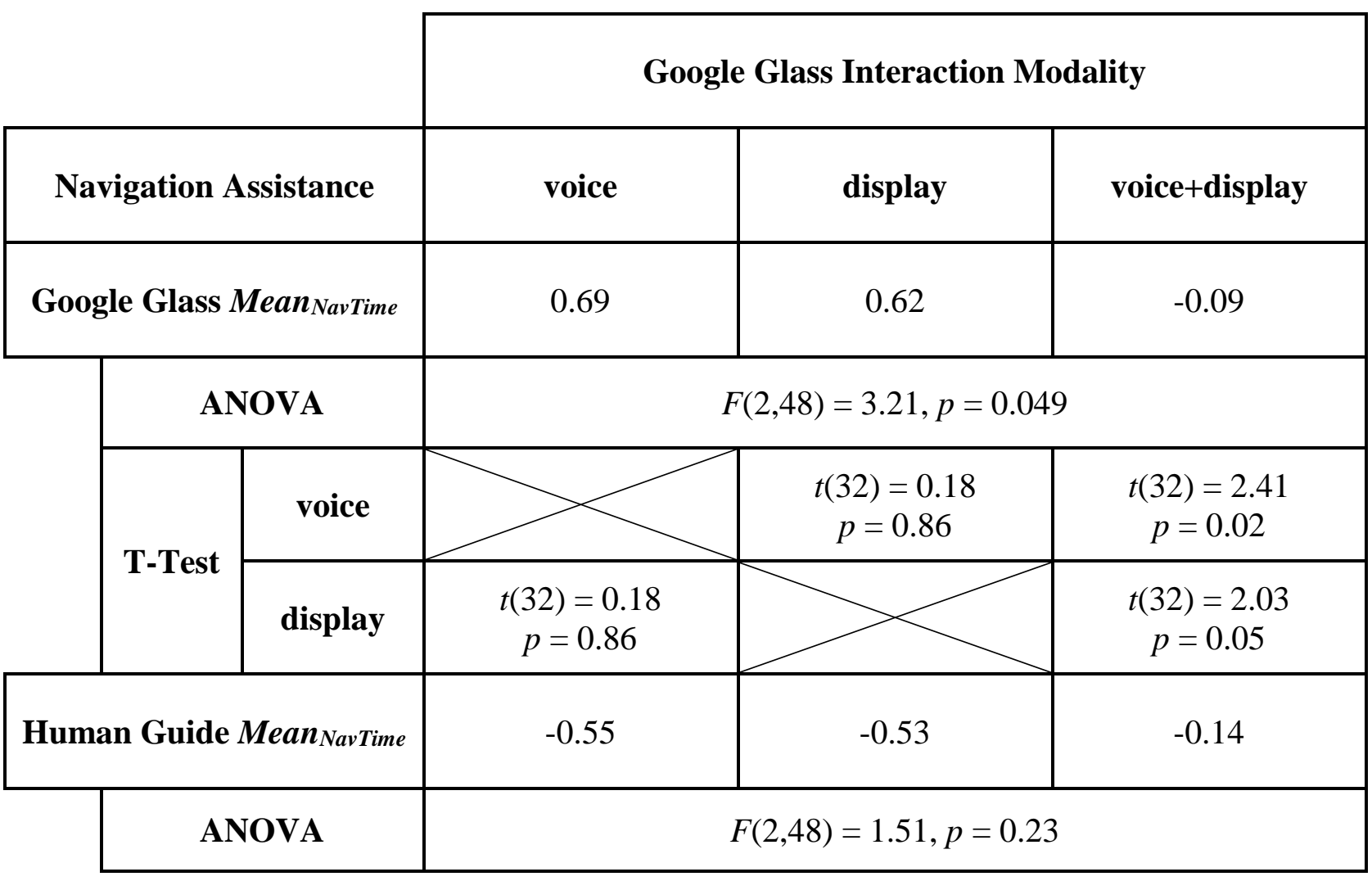

These results confirm only one of our hypotheses, namely, that the participants will need more time to navigate when guided by GG with display interaction modality. Our other hypotheses are not confirmed. Participants needed more time to navigate when guided by GG with voice modality, than when guided by HG. Also, we expected that with the voice+display modality participants will need more time for navigation when compared to HG; however, in 
this scenario the performance of both groups was similar. Thus, our hypotheses that display modality will show similar performance to voice+display one was not supported.

Table 8 shows the backtracking time t-test results between different types of navigation assistance. We found no difference between GG and HG assistance, when GG is operated with voice, or display modality. However, we found a significant difference between the GG and HG assistance, when GG operated in the voice+display modality.

Table 8. Backtracking Time T-test Results Between Different Types of Navigation Assistance.

\begin{tabular}{|c|c|c|c|}
\hline & \multicolumn{3}{|c|}{ Google Glass Interaction Modality } \\
\hline Navigation Assistance & voice & display & voice+display \\
\hline Google Glass Mean BackTime & -0.05 & 0.18 & 0.10 \\
\hline Human Guide Mean BackTime & -0.05 & 0.29 & -0.46 \\
\hline T-Test & $\begin{array}{c}t(16)=0.01 \\
p=0.99\end{array}$ & $\begin{array}{c}t(16)=-0.21 \\
p=0.84\end{array}$ & $\begin{array}{c}t(16)=3.61 \\
p=0.002\end{array}$ \\
\hline
\end{tabular}

Table 9 shows the backtracking time ANOVA results for different interaction modalities of Google Glass. We found no difference between the tested GG modalities with respect to backtracking performance. Also, no difference was found between the HG groups. Our hypotheses are only partially confirmed. We predicted that it will take longer to backtrack on routes at which GG, with display or voice+display modality, was used when compared to HG. However, only for the voice+display modality it took participants longer to backtrack. For the display and voice modalities, the backtracking time was similar to the HG. Therefore, our hypothesis regarding the voice modality is confirmed. 
Table 9. Backtracking Time ANOVA Results for Different Interaction Modalities of Google Glass.

\begin{tabular}{|c|c|c|c|}
\hline & \multicolumn{3}{|c|}{ Google Glass Interaction Modality } \\
\hline Navigation Assistance & voice & display & voice+display \\
\hline Google Glass Mean BackTime & -0.05 & 0.18 & 0.10 \\
\hline ANOVA & \multicolumn{3}{|c|}{$F(2,48)=2.34, p=0.79$} \\
\hline Human Guide Mean BackTime & -0.05 & 0.29 & -0.46 \\
\hline ANOVA & \multicolumn{3}{|c|}{$F(2,48)=2.50, p=0.09$} \\
\hline
\end{tabular}

\subsubsection{Correct Backtracking Decisions Performance}

Figure 21 shows the correct backtracking decisions made by the participants under different conditions. Also, Table 10 shows correct backtracking decisions t-test results between different types of navigation assistance. There was no significant difference between HG and GG with the voice modality, as well as with the display modality. However, when GG was used with the voice+display modality, the number of correct backtracking decisions was significantly lower than for HG. 
GG - Voice

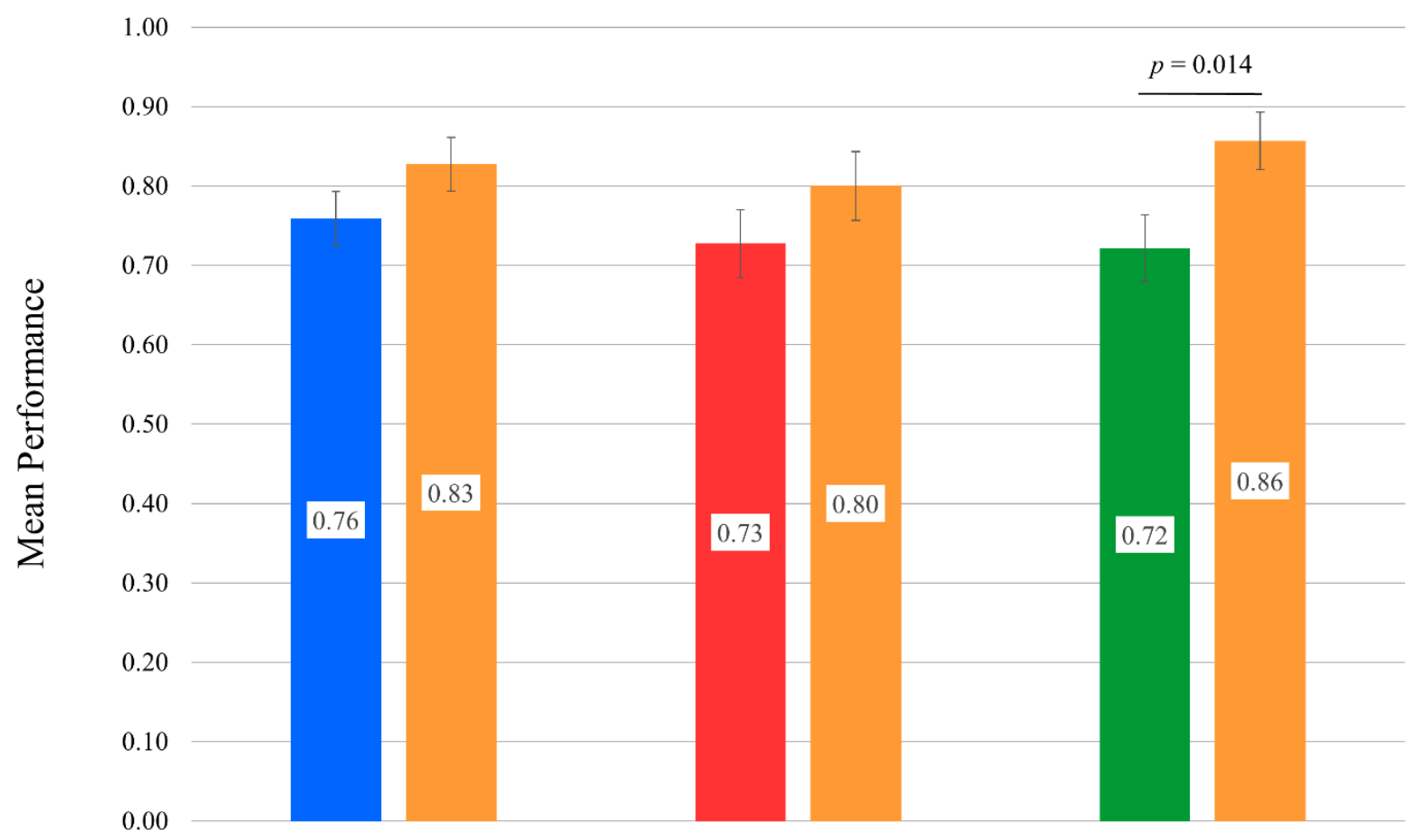

Correct Backtracking Decisions

Figure 21. Average number of correct backtracking decisions with the use of different types of navigation assistance and different Google Glass modalities. Error bars show standard errors. Sample size $n=51$ (17 participants in each group - GG-Voice, GGDisplay, GG-Voice+Display).

Table 10. Correct Backtracking Decisions T-test Results Between Different Types of Navigation Assistance.

\begin{tabular}{|c|c|c|c|}
\hline & \multicolumn{3}{|c|}{ Google Glass Interaction Modality } \\
\hline Navigation Assistance & voice & display & voice+display \\
\hline $\begin{array}{c}\text { Google Glass } \\
\text { Mean }_{\text {CorrectDecisions }}\end{array}$ & 0.76 & 0.73 & 0.72 \\
\hline $\begin{array}{l}\text { Human Guide } \\
\text { Mean }_{\text {CorrectDecision }}\end{array}$ & 0.83 & 0.80 & 0.86 \\
\hline T-Test & $\begin{array}{c}t(16)=-1.49 \\
p=0.16\end{array}$ & $\begin{array}{c}t(16)=-1.29 \\
p=0.21\end{array}$ & $\begin{array}{c}t(16)=-2.77 \\
p=0.014\end{array}$ \\
\hline
\end{tabular}


Table 11 shows correct backtracking decisions ANOVA results for different interaction modalities of Google Glass. The between-subject one-way ANOVA showed no difference between GG interaction modalities with respect to correct backtracking decisions. Also, no difference was found for different HG groups.

Table 11. Correct Backtracking Decisions ANOVA Results for Different Interaction Modalities of Google Glass.

\begin{tabular}{|c|c|c|c|}
\hline & \multicolumn{3}{|c|}{ Google Glass Interaction Modality } \\
\hline Navigation Assistance & voice & display & voice+display \\
\hline Google Glass Mean BackTime & 0.76 & 0.73 & 0.72 \\
\hline ANOVA & \multicolumn{3}{|c|}{$F(2,48)=0.25, p=0.78$} \\
\hline Human Guide Mean $_{\text {BackTime }}$ & 0.83 & 0.80 & 0.86 \\
\hline ANOVA & \multicolumn{3}{|c|}{$F(2,48)=0.57, p=0.57$} \\
\hline
\end{tabular}

Again, our hypotheses are only partially confirmed. As expected, there was no difference between GG with voice modality when compared to HG. Also, we predicted that participants who used GG with voice+display modality will make more backtracking mistakes when compare to HG. The results confirm this prediction. However, they do not confirm our hypothesis regarding the display interaction modality - GG participants should have made less correct backtracking decisions than HG users. The results show that the participants who used the display modality made similar number of correct backtracking decisions as participants guided by HG. 


\subsubsection{Visual Memory Performance}

Figure 22 and Table 12 show the participants performance in the visual memory test. There was no significant difference between $\mathrm{HG}$ and GG with any of the modalities: the voice modality, the display modality, and the voice+display modality.

Table 12. Memory Score T-test Results Between Different Types of Navigation Assistance.

\begin{tabular}{|c|c|c|c|}
\hline & \multicolumn{3}{|c|}{ Google Glass Interaction Modality } \\
\hline Navigation Assistance & voice & display & voice+display \\
\hline $\begin{array}{c}\text { Google Glass } \\
\text { Mean }_{\text {CorrectDecisions }}\end{array}$ & 0.62 & 0.61 & 0.67 \\
\hline $\begin{array}{l}\text { Human Guide } \\
\text { Mean CorrectDecision }\end{array}$ & 0.64 & 0.63 & 0.67 \\
\hline T-Test & $\begin{array}{c}t(16)=-0.56 \\
p=0.58\end{array}$ & $\begin{array}{c}t(16)=-0.46 \\
p=0.65\end{array}$ & $\begin{array}{c}t(16)=0.68 \\
p=0.95\end{array}$ \\
\hline
\end{tabular}

Table 13 shows the memory score ANOVA results for different interaction modalities of Google Glass. The between subjects ANOVA showed no significant difference between GG modalities with respect to the visual memory. Also, no difference was found between different HG groups. 
$\square$ GG - Voice

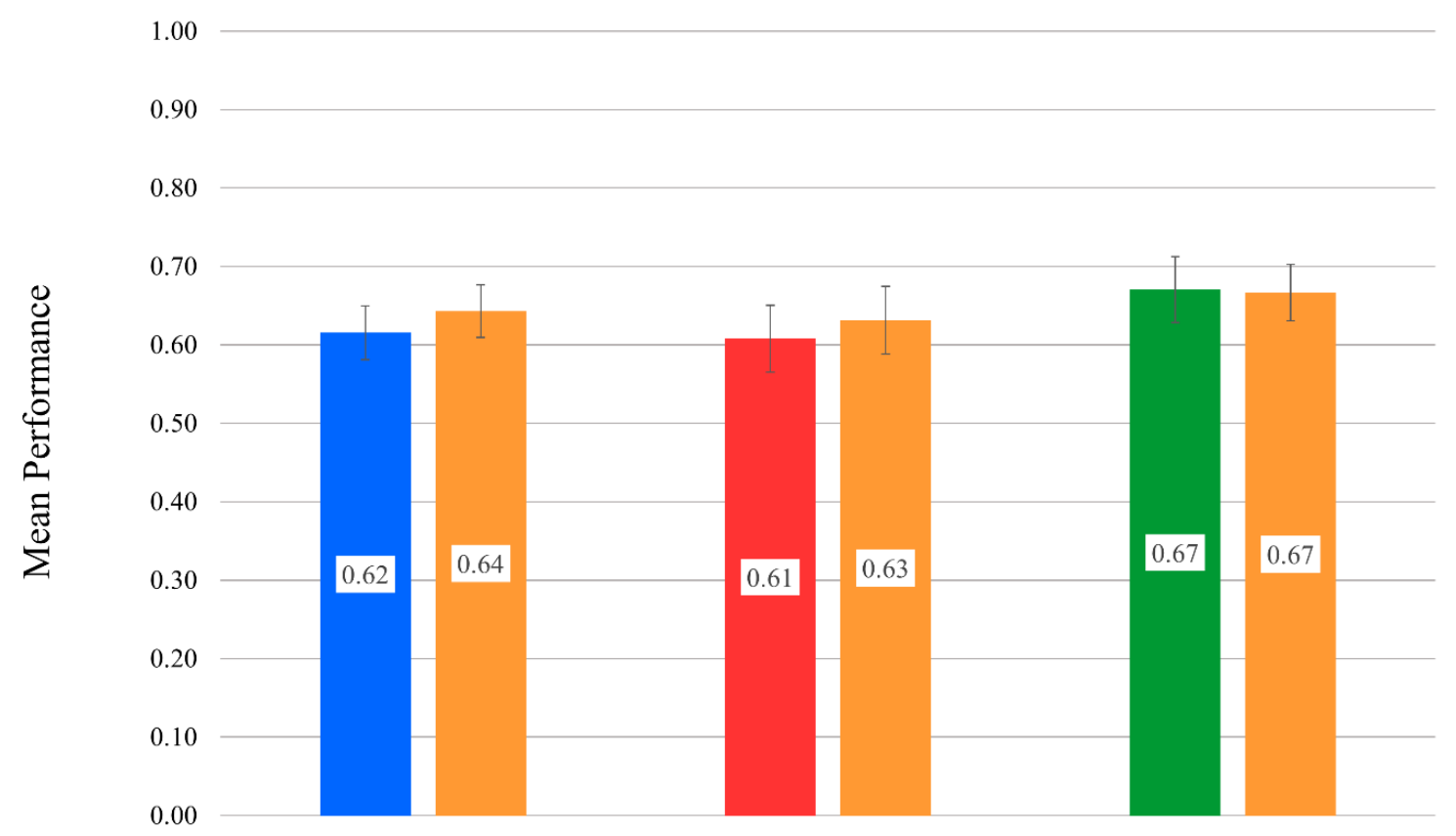

Visual Memory

Figure 22. Mean visual memory performance with the use of different types of navigation assistance and different Google Glass modalities. Error bars show standard error. Sample size $n=51$ (17 participants in each group - GG-Voice, GG-Display, GGVoice+Display).

Table 13. Memory Score ANOVA Results for Different Interaction Modalities of Google Glass.

\begin{tabular}{|c|c|c|c|}
\hline & \multicolumn{3}{|c|}{ Google Glass Interaction Modality } \\
\hline Navigation Assistance & voice & display & voice+display \\
\hline Google Glass Mean BackTime & 0.62 & 0.61 & 0.67 \\
\hline ANOVA & \multicolumn{3}{|c|}{$F(2,48)=0.62, p=0.54$} \\
\hline Human Guide Mean BackTime & 0.83 & 0.80 & 0.86 \\
\hline ANOVA & \multicolumn{3}{|c|}{$F(2,48)=0.20, p=0.82$} \\
\hline
\end{tabular}


For the visual memory score, only one of our hypotheses is confirmed, i.e. the users of GG with voice modality should remember similar number of encountered scenes when compared to HG users. Our other hypotheses are not supported. We expected that the participants will remember less scenes when guided by GG, with display or voice+display modalities, when compared to the participants guided by HG. However, they visual memory performance was not significantly different.

\subsubsection{User Experience}

We used Kruskal-Wallis test, with post-hoc Mann-Whitney or Wilcoxon analysis, for the subjective user experience evaluation of using different navigation systems (i.e., GG and HG) and different interaction modalities.

Figure 23 and Table 14 show how different interaction modalities affect user experience of using GG during navigation.

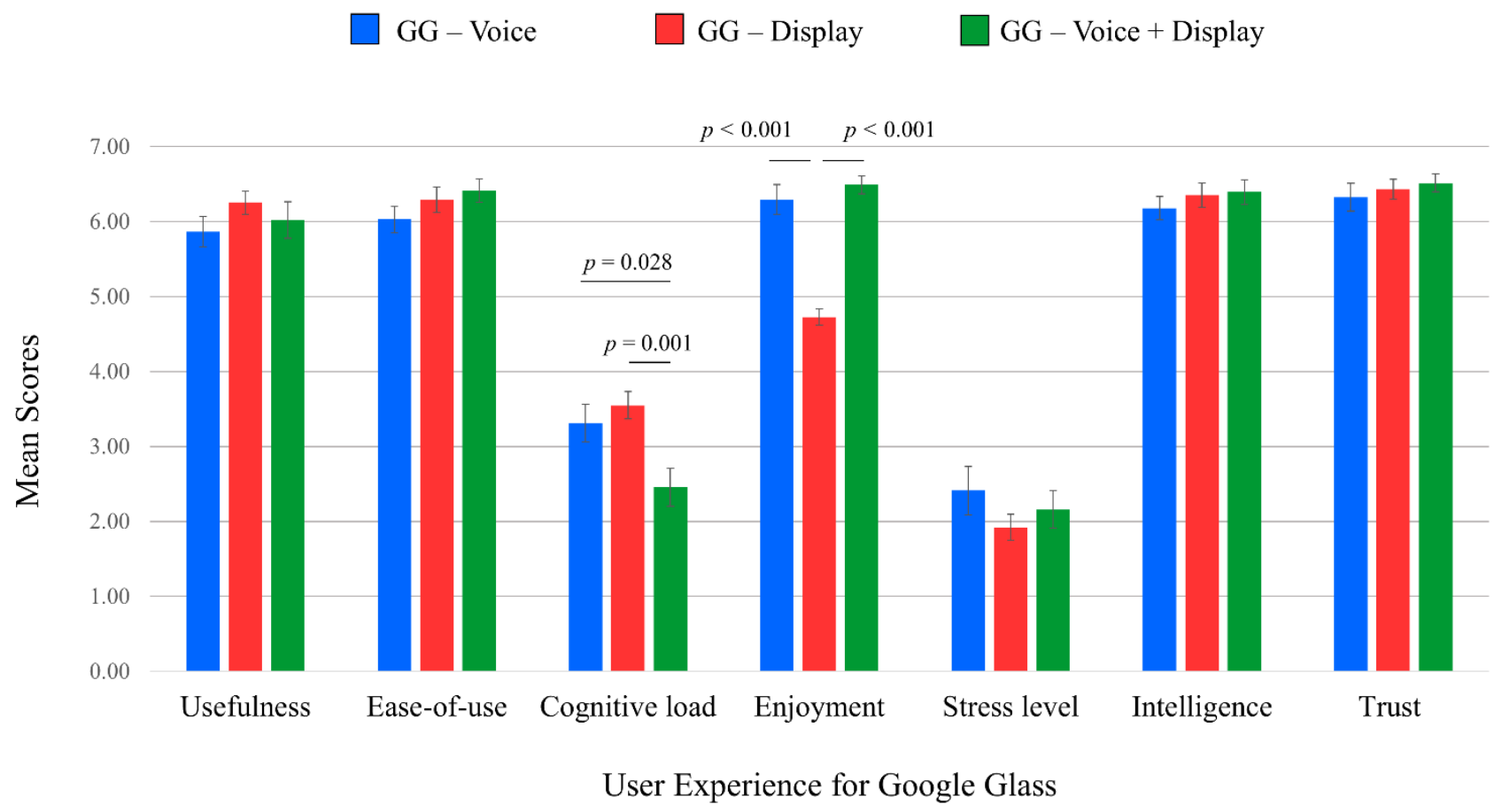

Figure 23. Comparison of user experience measures for different modalities of providing navigation information with Google Glass. Error bars show standard errors. Sample size $\boldsymbol{n}=\mathbf{5 1}$ (17 participants in each group - GG-Voice, GG-Display, GG-Voice+Display). 
We found significant difference for the measurement of cognitive load between different modalities. Post-hoc analyses showed significant difference in the perceived cognitive load between the voice and the voice+display modalities, as well as between the display and the voice+display modalities. There was no difference between the voice and the display modalities. Next, a significant difference, in the perceived enjoyment, was found for different GG modalities. The participants enjoyed GG with the voice modality more than with the display modality, also they enjoyed the voice+display modality more than just the display modality. There was no difference in the perceived enjoyment between the voice and the voice+display modalities. The participants, when guided by GG with different interaction modalities, perceived no significant differences for the following measurements: usefulness, ease-of-use, stress level, intelligence, and trust. 
Table 14. User Experience Kruskal-Wallis and Mann-Whitney Tests Results for Different Interaction Modalities of Google Glass.

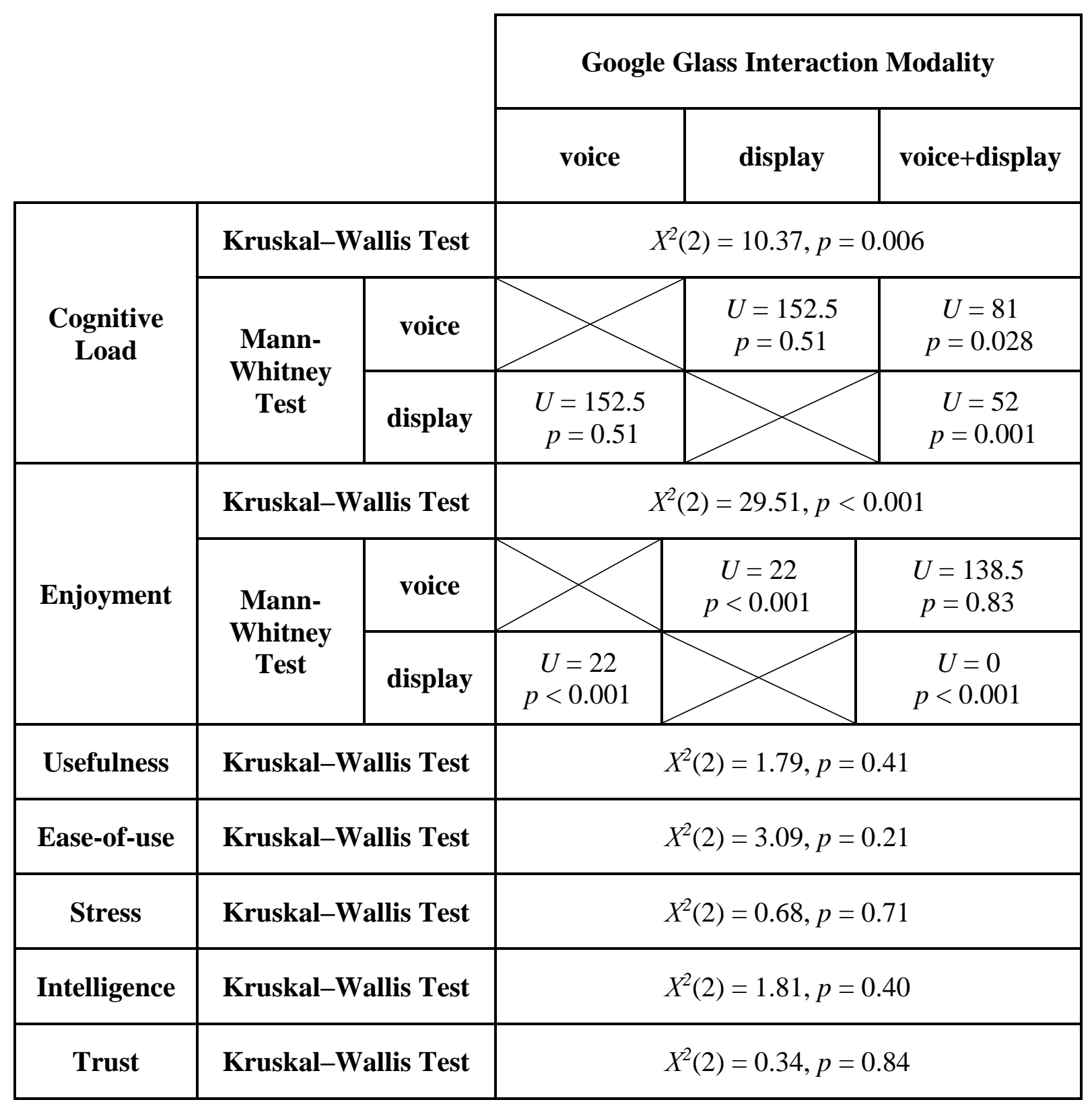




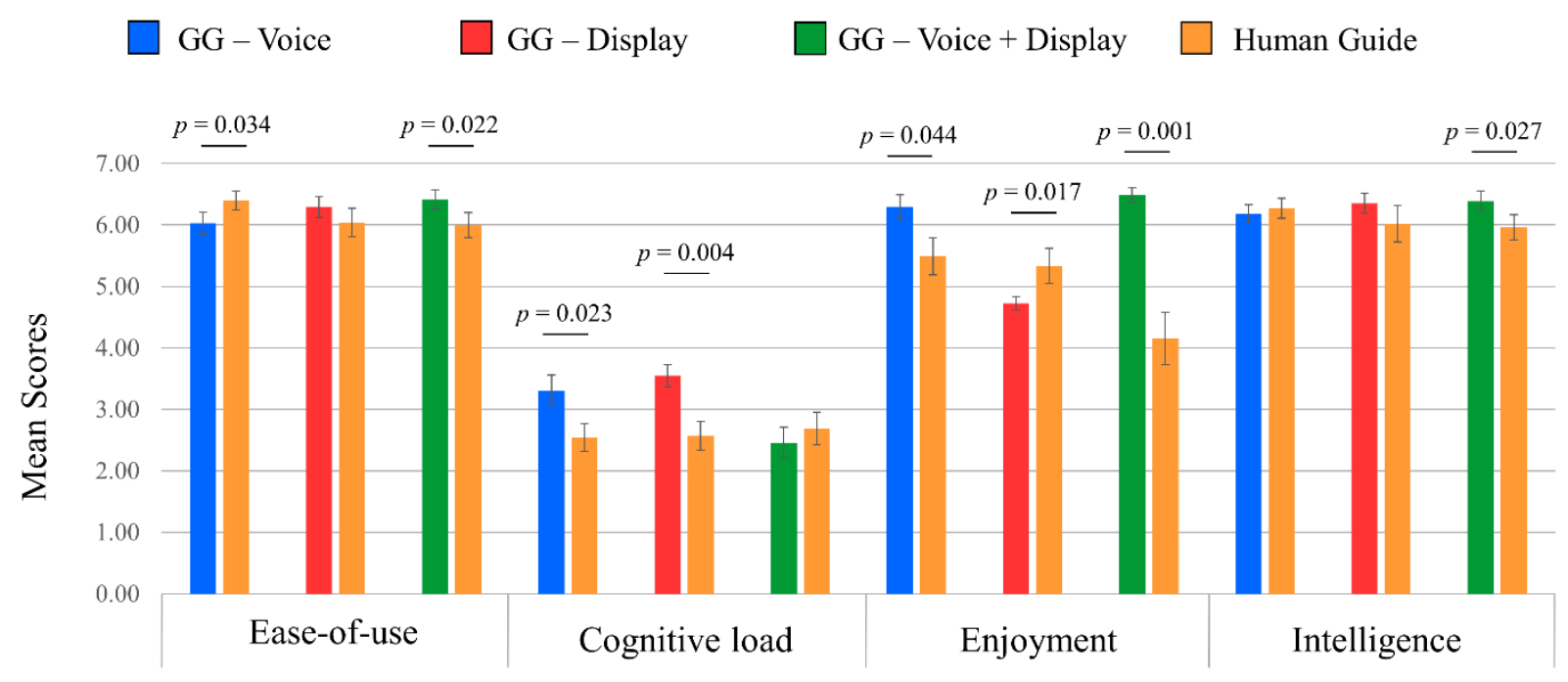

User Experience

Figure 24. Comparison of selected user experience measures for different types of navigation assistance and different Google Glass modalities. Error bars show standard error. Sample size $n=51$ (17 participants in each group - GG-Voice, GG-Display, GGVoice+Display).

Figure 24 shows subjective user experiences of measures for which significant differences were found between GG and HG assistances. Table 15 shows details for Wilcoxon tests results for user experience. The participants perceived HG as easier-to-use when compared to GG with the voice modality. However, when GG was used with the voice+display modality, the participants perceived it as easier-to-use than HG. There was no difference, in the perceived ease-of-use, for the display modality of GG when compared to HG. Also, we found no difference in the perceived ease-of-use between different groups guided by HG.

Next, the participants reported higher cognitive load while using GG with the voice modality than when guided by HG. This pattern was also evident for the display modality when compared with HG. Interestingly, when GG was used with the voice+display modality, the users perceived that cognitive load was lower, but not significantly, than for HG. Finally, no difference in the reported cognitive load was found between different groups guided by HG. 
Table 15. User Experience Wilcoxon Tests Results Between Different Types of Navigation Assistance.

\begin{tabular}{|c|c|c|c|}
\hline & Goog & ass Interaction & dality \\
\hline Navigation Assistance & voice & display & voice+display \\
\hline Google Glass Ease-of-use & 6.03 & 6.29 & 6.41 \\
\hline Human Guide Ease-of-use & 6.40 & 6.04 & 6.00 \\
\hline Wilcoxon Test & $\begin{aligned} Z & =-2.13 \\
p & =0.034\end{aligned}$ & $\begin{array}{c}\mathrm{Z}=-1.06 \\
p=0.29\end{array}$ & $\begin{aligned} \mathrm{Z}= & -2.29, p= \\
& 0.022\end{aligned}$ \\
\hline Google Glass Cognitive Load & 3.31 & 3.55 & 2.46 \\
\hline $\begin{array}{c}\text { Human Guide Cognitive } \\
\text { Load }\end{array}$ & 2.54 & 2.57 & 2.69 \\
\hline Wilcoxon Test & $\begin{aligned} \mathrm{Z}= & -2.28, p= \\
& 0.023\end{aligned}$ & $\begin{aligned} \mathrm{Z}= & -2.87, p= \\
& 0.004\end{aligned}$ & $\begin{array}{c}\mathrm{Z}=-0.96, p= \\
0.38\end{array}$ \\
\hline Google Glass Enjoyment & 6.29 & 4.73 & 6.49 \\
\hline Human Guide Enjoyment & 5.49 & 5.33 & 4.16 \\
\hline Wilcoxon Test & $\begin{aligned} \mathrm{Z}= & -2.01, p= \\
& 0.044\end{aligned}$ & $\begin{aligned} \mathrm{Z}= & -2.39, p= \\
& 0.017\end{aligned}$ & $\begin{array}{c}\mathrm{Z}=-3.33, \mathrm{p}= \\
0.001\end{array}$ \\
\hline Google Glass Intelligence & 6.18 & 6.35 & 6.39 \\
\hline Human Guide Intelligence & 6.27 & 6.02 & 5.96 \\
\hline Wilcoxon Test & $\begin{aligned} \mathrm{Z}= & -0.72, p= \\
& 0.47\end{aligned}$ & $\begin{array}{c}\mathrm{Z}=-0.90, p= \\
0.37\end{array}$ & $\begin{aligned} \mathrm{Z}= & -2.21, p= \\
& 0.027\end{aligned}$ \\
\hline
\end{tabular}


In terms of perceived enjoyment, the participants reported higher enjoyment when using GG with the voice modality than when guided by HG. Similarly, the participants enjoyed using GG with the voice+display modality more than listening to HG instructions. However, the participants enjoyed GG less than HG, when the display modality was used.

The perceived intelligence of GG was significantly higher than that of HG when the voice+display modality was used. When the voice modality is used the intelligence of HG is perceived as similar to GG. Also, for the display modality, HG intelligence is perceived as similar to GG. Finally, no difference in the perceived intelligence was found between different groups guided by HG.

Table 16 shows Kruskal-Wallis and Mann-Whitney tests results for significant differences in perceived user experience when guided by human. It is worth noting, that for the enjoyment measure, we found a difference between groups guided by HG. Reported enjoyment of being guided by $\mathrm{HG}$ is significantly higher for the scenario when the participants used GG with the voice modality, as compared to the scenario when they used GG with the voice+display modality. No significant difference is found in the reported enjoyment of using HG between participants who used GG with the voice or the display modalities, neither between participants who used the display or the voice+display modalities. 
Table 16. User Experience Kruskal-Wallis and Mann-Whitney Tests Results for Significant Differences in Perceived Human Guidance.

\begin{tabular}{|c|c|c|c|c|c|}
\hline & & & \multicolumn{3}{|c|}{ Google Glass Interaction Modality } \\
\hline & & & voice & display & voice+display \\
\hline \multirow{3}{*}{$\begin{array}{l}\text { Enjoyment } \\
\text { perceived } \\
\text { when guided } \\
\text { by HG }\end{array}$} & \multicolumn{2}{|c|}{ Kruskal-Wallis Test } & \multicolumn{3}{|c|}{$X^{2}(2)=6.47, p=0.039$} \\
\hline & \multirow{2}{*}{$\begin{array}{c}\text { Mann- } \\
\text { Whitney } \\
\text { Test }\end{array}$} & voice & & $\begin{array}{c}U=123.5 \\
p=0.47\end{array}$ & $\begin{array}{c}U=76.5 \\
p=0.019\end{array}$ \\
\hline & & display & $\begin{array}{r}U=123.5 \\
p=0.47\end{array}$ & & $\begin{array}{c}U=90 \\
p=0.059\end{array}$ \\
\hline
\end{tabular}

\subsection{Discussion}

In this section, we discuss how: 1) the two different types of navigation assistance (i.e., GG and $\mathrm{HG}$ ), and 2) the three interaction modalities, affect users' spatial cognition (navigation and backtracking performance), as well as visual memory and subjective user experience. Next, we analyse limitations of our experiment and propose future research directions.

\subsubsection{Visual Memory Is Not Affected by the Type of Navigation}

\section{Assistance or Interaction Modality}

The results show that the participants remembered a similar number of encountered visual scenes while using different types of navigation assistance (i.e., GG or HG) and different interaction modalities. These results indicate that none of the devices, neither any of the modalities, strongly affected the participants' visual attention. This confirms that people, in general, tend to remember the same visual scenes (Isola, Xiao, et al., 2011).Moreover, this result is in agreement with studies on human navigation, which show that different navigation systems (Krüger et al., 2004), or different modes of exploration (Gale et al., 1990; Richardson et al., 1999) do not affect landmark knowledge (i.e., landmark recognition). 


\subsubsection{Use the Voice+Display Modality for Best Navigation Performance,} but Be Aware of Its Drawbacks

Our results show that for most interaction modalities GG was inferior to HG as the navigation assistance. It took participants longer to navigate when they were guided by GG with the voice or the display modalities. However, when GG operated in the voice+display modality, the participants' navigation performance was as good as that achieved with HG.

We have expected that the voice modality of GG will realise HG's voice guidance condition such that the participants hear only voice commands. However, when guided by HG, the participants' find their way through the environment significantly faster than when guided by GG with the voice modality. Therefore, we conclude that the voice modality affected participants' navigation speed when compared to HG. This may be caused by a few factors. Firstly, the novelty of GG may affect participants' behaviour (Rogers, 2010). Secondly, the voice commands provided by GG was generated using voice synthesizer, which may require more efforts - resulting in higher cognitive load - from the participants when compared to commands given by an experimenter (Pisoni, 1997). Finally, the participants may walk slower, as they were focusing on auditory feedback in anticipation for the next navigation command from GG.

Next, we have expected that it will take longer to perform navigation using GG in the display modality when compared to HG. The results confirm our prediction. Most likely, slower navigation speed was caused by the fact that the participants have to shift their visual attention between the Glass display and the environment. However, at the same time, they have remembered a similar number of encountered scenes when guided by HG. This indicates that shifts of the visual attention did not affect participants' visual memory during navigation. 
Surprisingly, the participants guided by the voice+display modality of GG navigated through the environment as effectively as those guided by HG. We expected that with the voice+display modality participants will navigate slower, as they may need to shift their visual attention between the Glass display and the environment. Even if they depended solely on the voice commands, their navigation speed should be slower than that with HG - as indicated by the voice modality group results. Therefore, it is of special interest to analyse why the voice+display modality showed this result. The most plausible explanation can be obtained by combined interpretations of the results for the voice and the display modalities. Hence, the combination of both, the voice and the display modality, provides a redundant source of information to a user. Therefore, he or she can use both complementary information sources interchangeably. This information redundancy may bring a sense of robustness and hence confidence that a user will be able to receive a navigation command from at least one source (i.e., voice or display). Accordingly, this may reduce the user's stress, related to the anticipation for next command. In conclusion, the voice+display interaction users would be more confident during navigation and walk as fast as when they are guided by HG.

During backtracking, it took the participants similar amount of time to find their way to the starting location along routes at which they used GG with the voice or the display modalities as compared to routes at which they were guided by HG. Also, the number of correct backtracking decisions was similar for both the voice and the display modalities, as well as for corresponding $\mathrm{HG}$ routes.

However, along routes at which the participants used GG with the voice+display modality, it took them longer to backtrack when compared to HG. At the same time, we found no difference in the backtracking time between different interaction modalities of GG or different groups guided by HG. As presented above, GG with the voice+display modality provides navigation guidance which is as effective as $\mathrm{HG}$; however, this modality affects the 
backtracking performance when compared to HG. The most plausible explanation of this result is provided by the number of correct backtracking decisions. While backtracking, the participants made more mistakes along routes at which they were guided by the voice+display modality when compared to routes at which they were guided by HG. However, as presented above, backtracking mistakes were not caused by insufficient landmark knowledge (i.e., impaired visual memory, necessary for landmark recognition). The participants' visual memory was not affected by any of the interaction modalities when compared to HG. These results confirm that there is no correlation between the route knowledge and the visual memory of encountered scenes (Murakoshi \& Kawai, 2000). Therefore, the backtracking mistakes happened due to some impairment of the participants' spatial cognition. When using voice+display modality, the participants may depend fully on the navigation assistance. This could reduce direct engagement in environment exploration and indirectly negatively affects user's spatial cognition. Therefore, the participants acquired less spatial information, necessary for making correct backtracking decisions. As a result, it took them longer to backtrack as compared to HG.

\subsubsection{The Voice+Display Modality Is Perceived as Smarter and More Enjoyable to Use Than Human Guide}

This experiment also investigates user experience of using GG with different modalities. This was done by analysing the questionnaires filled by the participants.

The participants reported a higher enjoyment when using GG with the voice and the voice+display modalities when compared with HG. For the display modality, enjoyment was lower than for HG. Also, direct comparison of the three different interaction modalities showed that the participants enjoyed the voice and the voice+display modalities more than the display modality. This indicates, that information provided in the form of an auditory message make a system more enjoyable than when the display alone is used. Moreover, as 
the voice+display modality outperformed HG $(p=0.001)$ in terms of enjoyment, it is recommended to use both sources of information to maximize users' positive experience.

For the perceived ease-of-use, we found no difference between different interaction modalities. However, when compared with HG, we noticed that the participants considered the voice modality as harder to use than HG. It would be expected, as it is easier to understand experimenter's voice than voice generated by a voice synthesizer. Surprisingly, the voice+display modality was considered as easier to use when compared to HG. This may be explained by the fact that this modality provides navigation command from two sources (i.e., voice and display). Therefore, it should be easier for a participant to process information, as he or she can use multiple senses to do it. Again, this result favours the use of the voice+display modality.

Next, the participants perceived GG with the voice+display modality as more intelligent than HG. This result is contradictory to previous studies, in which HG was considered as the most intelligent navigation system (Xu et al., 2014). However, as presented above, this contradiction can be explained by the fact that GG is perceived as more intelligent when it is providing navigation with use of multiple modalities (i.e., voice and display); while HG uses only voice commands.

Finally, results showed that participants perceived higher cognitive loads when using GG in the voice and the display modalities when compared to HG. This may indicate that the participants' cognitive load is higher when they need to process auditory stimulus generated by the voice synthesizer when compared to an experimenter's voice (Fernández-Torné \& Matamala, 2015). Also, cognitive load is higher when the navigation is provided on the Glass display when compared to experimenter's auditory instruction. This may be caused by participant's visual attention switches - between the Glass display and the environment. However, with the voice+display modality, there is no difference in the perceived cognitive 
load between GG and HG. Also, for the voice+display modality, perceived cognitive load is lower than for the voice or the display modalities. Again, these results may be explained by the fact that the voice+display modality provides two sources of information. This may reduce participants' cognitive load, as they can use both complementary information sources interchangeably, which reduce the fear of missing necessary information and raise their confidence in the guidance.

During indoor navigation, user demands easily understandable navigation information that prevents him or her from disorientation (Hegarty, Montello, Richardson, Ishikawa, \& Lovelace, 2006). Moreover, a navigation assistance should provide information in a way that keeps the level of cognitive load low when a user interprets, and applies navigation information (Ohm, Müller, \& Ludwig, 2016). In general, we found that GG can be as effective as HG during guided indoor navigation. Moreover, with the voice+display modality, GG is perceived as easier to use, more enjoyable, and smarter than HG. Meanwhile, it induces equivalent cognitive load. In summary, GG, with the voice+display modality, seems to be a practical solution for real-life navigation assistance.

\subsubsection{Limitations and Future Research Directions}

Although our experiment provides valuable information about the use of GG as indoor navigation assistance, we are fully aware of its limitations. We present possible areas for improvement and potential directions for future studies.

Our study compared GG assistance only against HG. In the future, GG assistance should be evaluated against other indoor navigation systems (e.g., mobile phone, 2D map, 3D route planning (van Schaik et al., 2015)). Also, future studies should use a control group(s), which will navigate with use of pre-installed indoor signage system, without any additional support. This will allow us to investigate how navigation assistance may benefit a user, and how it affects spatial cognition when compared to unguided navigation. 
Also, it would be of interest to see how individual preferred navigation styles (e.g., 2D map, voice information, visual clues, etc.) correlate with different types of navigation assistance and different navigation modalities. Therefore, future studies should include such independent variable - collected through a questionnaire or a short test, which would present available navigation aids and modalities.

Our system was implemented as a mock-up solution - Google Glass application controlled by an experimenter, not an automated one. This was necessary for the experiment setting, as we focused on how the navigation assistance affects users' spatial cognition while ensuring that the system is reliable, precise and consistent. However, mock-up systems do not fully model real solutions. Therefore, additional user validation, with a real system implementation, would be needed for the development of a commercial navigation assistance based on GG.

Moreover, due to time constraints of the experiment, we evaluated the systems on two routes. Future studies may replicate our experiment on a larger number of routes, use more complex ones, or apply different navigation scenarios (e.g., shopping malls, schools, hospitals, etc.).

Next, we can improve the current system by incorporating additional information about the next decision point ahead of time. Also, some distance information that would allow a user to anticipate route changes during navigation may be beneficial to increase his or her "feel of control and accomplishment". For example, there might be a voice message that informs a user the distance to the next turning point. Also, the display information might be presented continuously, with a direction arrow that changes colour and/or shape when a user approaches the next decision point.

A user should perceive an assistive tool as trustworthy enough to follow its instructions. Our results show that the participants have trusted GG, in all modalities, to an 
equivalent level as they have trusted HG. However, concerns related to trust, especially towards novel wearable devices, should not be ignored or omitted from the research agenda as it might cause acceptance barriers in some user groups (e.g., elders) or in other application contexts (e.g., mission critical navigation).

Indoor navigation assistance is especially important for people with cognitive impairment in navigation abilities, like: dementia, inability to localize oneself in an environment (Corkin, 1984), with difficulties in long-term planning (Luria, 1966), or with degraded cognitive processes (Rau \& Wang, 2003). It should be noted, that the participants recruited in this study are relatively young, and may not be representative of potential users (e.g., elderly, with mild cognitive impairment). Therefore, future studies should support our findings with a broader range of potential users. For example, the system could be evaluated in a situation of an elderly person who needs to find his or her way to a bank cashier in a new public building. 


\section{Towards a Novel Cognitive-Inspired Indoor Navigation System}

This chapter review cognitive systems that mimic human visual memory or navigation abilities. These systems play an important role as a basis for building cognitive-inspired, autonomous navigation systems. Next, we propose a novel, wearable, cognitive-inspired navigation system. The system is able to extract a topological representation of an explored environment and to provide efficient guidance through already mapped or pre-defined places. The topological structure is represented by visual scene categories (nodes of the topology) and their spatial relations (edges between nodes). To the best of our knowledge, it is the first design and demo of such a system. The proposed system design was evaluated, in Matlab ${ }^{\mathrm{TM}}$, with real data collected with Google Glass. This system provides a strong foundation for efficient, real-life, navigation solutions that may be deployed in a number of scenarios (e.g., shopping malls, airports, offices, etc.).

\subsection{Cognitive-Inspired Systems for Navigation with Visual Information}

\section{Processing}

The main task for cognitive-inspired system is to model human performance in different task situations (Duch, Oentaryo, \& Pasquier, 2008). Newell (1994) provided 12 criteria for the evaluation of cognitive systems: adaptive behaviour, dynamic behaviour, flexible behaviour, development, evolution, learning, knowledge integration, vast knowledge base, natural language, real-time performance, and brain realization. We can categorize cognitive systems into three main groups based on the different memory models used: symbolic, emergent, and hybrid architecture. Symbolic systems use rule-based or/and graph-based representation of memory. In emergent systems, globalist memory and localist memory are used. For hybrid systems, localist-distributed or symbolic-connectionist memory model is used. In Table 17 the taxonomy of different memory types used in cognitive systems is presented. 
Table 17. Memory types used in different cognitive systems.

\begin{tabular}{|c|c|c|}
\hline Symbolic system & Emergent system & Hybrid system \\
\hline Rule-based memory & Globalist memory & Localist-distributed memory \\
Graph-based memory & Localist memory & Symbolic-connectionist memory \\
\hline
\end{tabular}

Numerous cognitive-inspired systems have been proposed for human cognition, ranging from perception, decision making, to action and control. These systems play a vital role as foundation for building intelligent systems, whose capabilities may one day be similar to that of the human brain. In the following sub-sections, we present selected cognitive systems that mimic human navigation abilities.

Figure 25 presents basic modules of multiple cognitive systems. The external environment is perceived by different sensors (e.g. cameras, microphones, etc.). Short-term memory is responsible for storing data necessary for current task. Long-term memories, declarative and/or procedural, store information necessary to support short-term memory. Those memories acquire knowledge through learning processes, declarative and/or procedural. Working memory cooperate with procedural memory, when the most suitable action for a particular situation needs to be selected. This action is then executed, either within the cognitive system (e.g., update system's working memory), or in the external world (e.g., display information). 


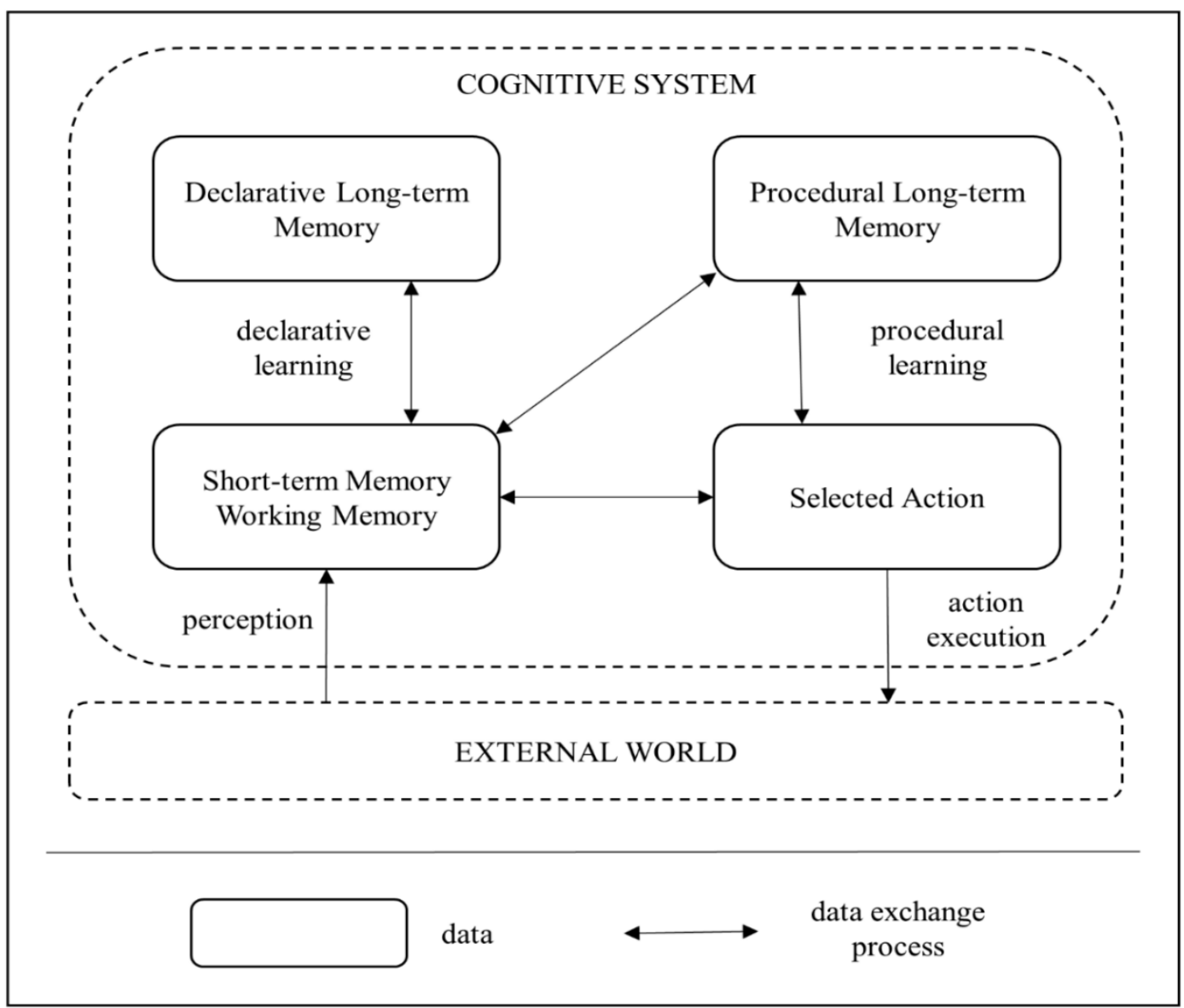

Figure 25. Most common structure of cognitive system. Based on Samsonovich (2010).

\subsubsection{DeSTIN}

DeSTIN is an emergentist cognitive system. This system addressed the problems of general intelligence by using hierarchical spatiotemporal networks. So far practical use of DeSTIN has focused on visual and auditory processing. This system allows for powerful unsupervised classification of different visual inputs (Goertzel, Lian, Arel, de Garis, \& Chen, 2010). It can create structures adequate for different categories of objects located in the scenes (categories like: beds, lamps, pets). This system is also capable of creating categories for actions (e.g. reaching, falling) which it sees (Goertzel et al., 2010). 


\subsubsection{NOMAD}

NOMAD (Neurally Organized Mobile Adaptive Device) is another emergent system. It is also known as Darwin automata. The main task of this system is pattern recognition in real time. NOMAD is equipped with vision sensors, as one of the inputs to the system. As for now this cognitive system is controlled by simulated nervous system $\left(\sim 10^{5}\right.$ neurons with $\sim 10^{7}$ synapses) (Duch et al., 2008), which run on powerful computers. One of task of Darwin automata systems VI and VII is the ability for invariant visual object recognition.

\subsubsection{DUAL}

DUAL is probably the most impressive hybrid system. This system uses both symbolic and neural network mechanisms. This combination can be considered to be DUAL's most significant feature. Nestor and Kokinov (2004) present model of DUAL system where they connect raw visual input with systems semantic memory, this model takes advantage of this hybridity by combining massively parallel activation-based computations with a serial attention-based symbolic processing mechanism instantiating the principle of active vision. Due to this change, DUAL becomes capable of processing its visual input.

\subsubsection{ViSTARS}

ViSTARS (Visually-guided Steering, Tracking, Avoidance, and Route Selection) is a biologically inspired system of the dorsal ("where") visual pathway in the primate brain (Goodale \& Milner, 1992). The system simulates hierarchical processing in the dorsal pathway, taking as input image sequence. It computes estimates of the position, speed, and heading of objects (i.e. obstacles) relative to the camera, and then uses these to compute the steering behaviour required to avoid the objects. In other words, the model transforms stream of raw visual input into ingredients needed for navigation, while also performing basic obstacle avoidance, rather than full navigation. 
The model does not possess a spatial map, although such a map could be constructed from the model's outputs. The model also does not perform localization (i.e. estimation of current position relative to known coordinates), but it could potentially be combined with a model of the brain's ventral ("what") visual pathway (Goodale \& Milner, 1992) to perform landmark recognition for triangulating position. In terms of memory, the model does not implement memory per se, although the mathematical equations it uses for smooth steering can be considered to be a form of procedural (implicit) spatial or motor memory. Nonetheless, the model's outputs form an essential part of visuo-spatial memory.

\subsubsection{GridPlaceMap}

GridPlaceMap is a cognitive system of the computational mechanisms that give rise to the spatial properties of "grid" and "place" cells from estimates of heading direction and linear motion speed. In particular, this system combines computations for path integration (McNaughton et al., 2006) with learning rules for self-organizing maps, and reproduces both the grid-like firing fields of grid cells and the localized firing fields of place cells. In other words, the system simulates the process of localizing current position from other navigational raw ingredients. In some sense, GridPlaceMap provides the next stage after ViSTARS, in terms of the computational pipeline that begins with raw pixel images and ends with navigation behaviour. ViSTARS converts a pixel stream into estimates of position and velocity of external obstacles, while GridPlaceMap converts self-motion velocity into estimates of self-position. The former deals with external objects with respect to the camera, while the latter deals with the camera with respect to an allocentric coordinate system, but in theory these two systems can be combined (with minor modifications).

GridPlaceMap can be considered to be a form of abstract spatial memory, since it generalizes grid and place firing fields from an episodic-like set of learning trials. This system can generate representation for many locations, store them, and automatically update 
current one. However, visual memory may play crucial role in supporting this system, by providing a confirmation signal for the calculated location.

\subsubsection{Leabra}

The Leabra (Local, Error-driven and Associative, Biologically Realistic Algorithm) cognitive system focuses on the nature of learning, combining error-driven and Hebbian associative mechanisms. Its neural network algorithm combines a biological theory of neuron function with learning (Jilk, Lebiere, O’Reilly, \& Anderson, 2008; O'Reilly, 1996).

Herd and O'Reilly (2005) presented a parallel neural network model of visual search based on the Leabra framework. The model consists of several different layers. The retinotopic feature layer, which corresponds to early ventral visual stream areas, contains the representation of a specific feature in a specific location. The location layer, which corresponds to late dorsal visual stream areas, contains the representation of any feature at a specific location (Herd \& O'Reilly, 2005). An additional template layer simulates a top-down signal that represents the features of the search target.

A visual module based on the Leabra algorithm can store knowledge as patterns of neural firing and synaptic strengths. Therefore, this module is capable to store implicit visual memory, which provides mechanisms of bottom-up perception for recognition of entities in the environment. The output of this system can be used for creating explicit visual memories. These memories can be transformed to symbolic representations, which can be easily processed by high-level cognitive system. This neural network structure can be used as a recognition module in a more complex system and provide basic information about visual input.

\subsubsection{ART}

ART (Adaptive Resonance Theory) has been introduced by Stephen Grossberg in 1976 and developed since then (Grossberg, 2012). ART is one of the most important attempts in 
simulating biologically inspired incremental learning. This theory tries to explain how brain learns objects and events in continuously changing word. It may be considered as blueprint for higher-level data processing in general navigation system.

ART merges new data with existing memory by checking for similarity between this new data and data already stored in the memory (neural network). If there is a close match, new data is assimilated with existing memory. Otherwise, this new data is stored as a new memory, with neurons and connections added to the network. The first ART system - ART1 - processes only discrete input and ART2 deals with continuous input. While these two systems provide only unsupervised learning mechanism, supervised mechanism was added to the ARTMAP model.

\subsubsection{ACT-R}

ACT-R (Adaptive Control of Thought - Rational) system contains independent modules that model different brain regions (John R. Anderson, 2007; John R. Anderson et al., 2004). The ACT-R consists of the following modules: the intentional (goal), imaginal, declarative, action, visual, and procedural (pattern matcher). The pattern matcher is considered to be the central component of the cognitive system. It searches for the production rule that best matches the current state in the working memory buffers. The selected production is executed ("fires"), which may cause changes in the system state. This mechanism can be compared to human procedural memory, in which information about task execution is stored (e.g. how to ride a bicycle, how to push keyboard buttons). Long-term memory in ACT-R is organized as a single module (declarative memory). Both procedural and declarative memories are permanent, without forgetting mechanisms (Kennedy \& Trafton, 2007). Knowledge about previous states (episodic memory), symbolic representations of objects, and the spatial relations between them is also stored in form of chunks. Each chunk has its own activation level, which reflects its usefulness in the past and 
its current meaningfulness. A chunk's activation level affects its probability of being retrieved to procedural memory, mimicking the way that people recall important or recent memories more easily than remote or less significant ones.

ACT-R has two visual modules (visual-location and visual-object). The visuallocation module contains locations of all objects, while the visual-object module provides information about a particular object as chunk. One drawback of ACT-R's visual processing is that so far it has been used only for images in which there is no noise, blur, distortions, etc. ACT-R encodes all object locations in a reference system that is relative to the screen edge. System called ACT-R/S enriches object representation to an egocentric point of reference (Harrison \& Schunn, 2003). Winkelholz and Schlick (2007) propose the extension for ACT$\mathrm{R}$, which provides possibility to encode object-location in a real allocentric local reference system. All these systems present relations between entities in two dimensions. By enriching ACT-R with a module capable of egocentric and allocentric object representation both in two and three dimensions, this system may gain realistic model of visual-working memory, which will also allow creating long-term memory chunks. This cognitive system may be used for processing high-level tasks during navigation.

\subsubsection{SAL}

SAL (Synthesis of ACT-R and Leabra) is hybrid system (Jilk et al., 2008). The ACT-R visual module responsible for object identification is replaced with a Leabra neural network vision module, allowing processing of raw bitmap images in a way impossible for the standalone ACT-R system. This new solution has been used in a navigation-search task in a virtual environment. The ACT-R part of this system is responsible for navigation, as it stores all navigation points in declarative memory. The Leabra visual module is responsible for object recognition, and for providing the symbolic form of it. SAL can navigate in virtual environments if navigation points are already encoded in the system. Thanks to this synthesis, 
SAL can accomplish tasks that are impossible for either ACT-R or Leabra alone. This shows that integration of different cognitive systems may lead to more powerful and flexible solutions.

\subsubsection{SOAR}

SOAR (Laird, 2012) is a cognitive system that models the interaction between working, procedural and episodic memory. Procedural knowledge is represented as "production rules". When the required conditions for a rule matches the contents of working memory, the rule "fires" and updates working memory. SOAR's episodic memory consists of snapshots of working memory (Nuxoll \& Laird, 2007). It is retrieved by finding the episode that best matches a given cue in working memory. The retrieved information is put into a specially designated area of working memory, so that past and present information can be distinguished. The system does not include mechanisms of memory consolidation, forgetting, generalization across episodes, etc.

Lathrop, Wintermute, and Laird (2011) extend SOAR by adding SVS (a Spatial/Visual System). This extended system includes a visual buffer for depictive representations (e.g. shape, texture) and a "spatial scene" entity for quantitative representations (e.g. precise object locations). These two entities allow for pictorial and mathematical manipulation of visuo-spatial information respectively. Symbolic manipulation is already enabled by the existing SOAR system. SVS does not include a front-end that performs low-level vision. Rather, SVS is used in virtual environments that already contain the quantitative spatial representations used for mathematical manipulation. Overall, SOAR and its extensions operate at an intermediate stage - SOAR does not process pixel images, but performs some additional processing on the navigational raw ingredients.

SOAR has not been used for navigation purpose, but its symbolic decision-making capabilities could be extended to this domain. The mathematical visuo-spatial manipulation 
and reasoning capabilities of SVS could be used to support triangulation of location, comparing planned and actual routes, etc. SOAR is a well-known, well-developed cognitive system; therefore, it should be considered as one of the main candidates for the control component of cognitive-inspired navigation system.

\subsection{Challenges Faced by Cognitive System for Navigation with Visual Input}

The space of possible cognitive systems that can be used for navigation is very large. At the levels of system, representation and processes, there are many design decisions that have to be made. At the architectural level, how should spatial and visual memory be implemented, in relation to episodic and semantic memory? At the representational level, should spatial representations be allocentric, egocentric, or both? How much visuo-spatial detail should long-term episodic memories contain? Should the world be represented as a multi-scale hierarchy of maps, or as a linked mosaic of detailed maps? At the level of computational processes, how are detailed, contextual episodic memories combined into abstract, contextfree semantic memories? These are just some of the issues that any cognitive system for navigation with visual input has to address.

In the above short review of cognitive-inspired systems, which model different functions needed for navigation, we presented their strengths and weakness. Table 18 presents the navigation functions that each of reviewed cognitive system can perform in the most efficient way. None of the current cognitive systems alone can automatically map an indoor environment, and to provide navigation based on the collected data. However, by combining current cognitive systems one should be able to obtain a coherent navigation system. This new system should be able to provide efficient navigation in outdoor as well in indoor environments. For example, in Figure 26, we provide a sketch of how different cognitive systems can collaborate during navigation task. ViSTARS computes direction and velocity of moving objects, which allows for short distance navigation. It also provides input 
to GridPlaceMap, which helps to estimate current location. However, this location should be confirmed by at least one more redundant module (e.g. by using visual memory to recognize landmarks). Next, a subsequent intermediate navigation target is selected by high-level cognition modules (e.g. parts of ACT-R or Soar architectures). The visual recognition module (e.g. Leabra) provides recognition of entities present in the visual input. Its output is used by the visual memory module, which tries to match elements of the current scene to prior visual knowledge (both specific instances and general visual schemas). If a match occurs, the visual memory module can pass on the information to spatial, semantic and episodic memory modules. These are then used to support navigation, or to provide information requested by the user (e.g. "Isn't that my friend's car ?"). This is just a brief and simple example of how different cognitive systems can collaborate to produce navigation, and is not meant to be a complete (or the only possible) system description.

Nevertheless, motivated by research in visual memory, human navigation, and cognitive-inspired systems in general, as well as the research findings of our experiments related to this challenging problem, we have concentrated our work on designing and implementing a novel, cognitive-inspired visual indoor navigation system, not on combining already existing cognitive systems.

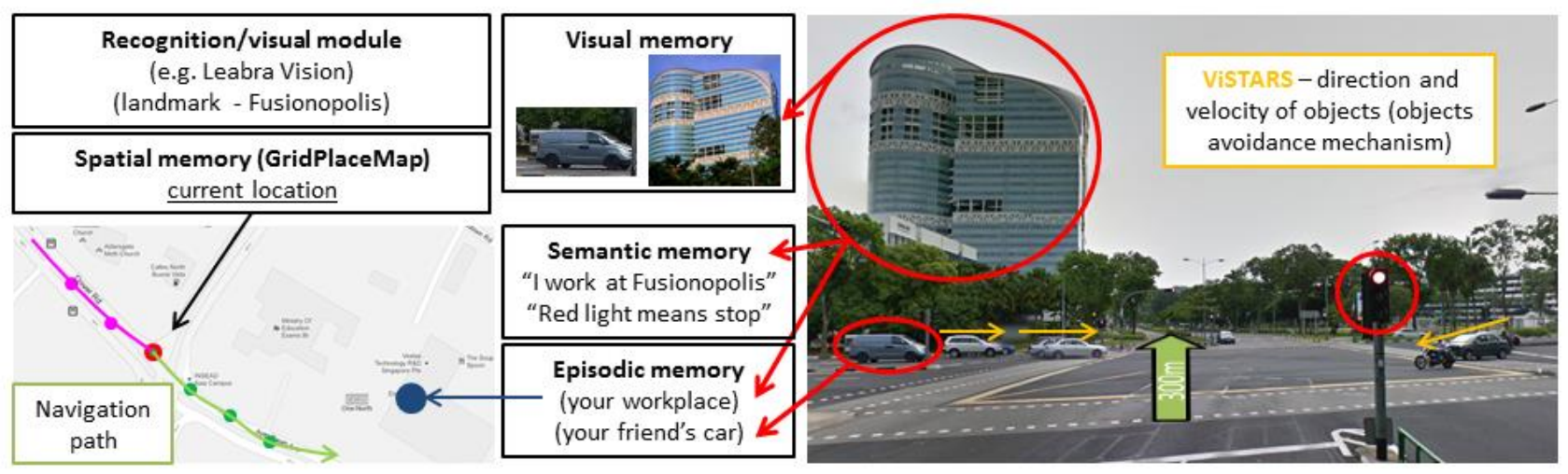

Figure 26. Example of different cognitive architectures cooperation during navigation. 
Table 18. Possible navigation functions provided by various cognitive systems.

\begin{tabular}{|c|c|}
\hline Cognitive System & Navigation Function \\
\hline DeSTIN & classification of different visual inputs \\
\hline NOMAD & visual object recognition \\
\hline DUAL & efficient processing of visual input \\
\hline ViSTARS & objects avoidance \\
\hline GridPlaceMap & representing location by place cells \\
\hline Leabra & landmarks recognition \\
\hline ACT-R & higher-level cognitive support \\
\hline SAL & avigation in familiar environments \\
\hline SOAR & long-term memory for visited places \\
\hline ART & higher-level data processing \\
\hline
\end{tabular}

\subsection{Novel Cognitive-Inspired Indoor Navigation System}

As mentioned in the previous section, the proposed novel, cognitive-inspired, indoor navigation system is able to automatically map an indoor environment, localize a user within already mapped environments, and therefore, it is also able to provide guidance instructions. To the best of our knowledge it is the first design and demo of such a system. First, we explain why we claim that the system is cognitive-inspired. Next, we present tools and algorithms used for the system design. Then, we show how the navigation system is able to create topological map of an environment. Finally, we present sample guidance process using our system and predefined topological structure of an environment. As a proof-of-concept, of our system, was implemented and tested in the Matlab ${ }^{\mathrm{TM}}$ environment. With further improvements to the design and algorithms proposed in our system, the system has potential to be deployed as a real-time, reliable, and satisfactory indoor navigation assistance. 


\subsection{Cognitive-Inspired: Combine Egocentric and Allocentric Environment}

\section{Representations}

We design the cognitive-inspired system based on current knowledge of human navigation, both from cognitive neuroscience and psychology findings. Cognitive-inspired, our system combines egocentric and allocentric representations of an environment to emulate human cognitive process for navigation assistance. Egocentric representation is obtained from a camera (i.e., video), and mimics human visual system. Allocentric representation is obtained from accelerometer and gyroscope data. These data provide information about user's movements - it mimics human vestibular system, and is used to create nodes that represents human brain place cells (Doeller et al., 2012). While similar information may be obtained from an optical flow extracted from the video, which is less reliable and more computational intensive; thus, we have decided to use accelerometer and gyroscope. By combining the egocentric and allocentric approach our system created a topological representation of an environment, which is similar to human landmark and survey knowledge of a surrounding.

In perspective of common cognitive systems design, our system perceives an external environment by camera, as well as accelerometer and gyroscope sensors. Short-term memory of our system is represented by current egocentric and allocentric representations of an environment. In our system, long-term memory is represented as a topological structure of already mapped environment (or pre-load data). During navigation, a particular action is selected based on the topological structure and specified destination location. This action is then executed and can be presented to a user.

Algorithm 1 presents general (high-level) design of our navigation system. The process starts with loading pre-defined topology of the environment. Current location and user orientation needs to be provided beforehand. Next, the final location (i.e., goal) is defined by the user, in our demo this location is always defined as the last available node in 
the topology structure. Thereafter, our system prepares the navigation path, the sequence of nodes that have to be travelled in order to achieve the goal location. For our proof of concept implementation, the navigation path is defined as subsequent nodes in the topology structure - as created during the environment mapping process. Then, our system in the loop (Algorithm 1, lines 6-15), defines the next node that should be reached by the user, and provides navigation command accordingly to the action needed to reach this node. The loop continues until the final node is reached. Within the loop, the video input from the camera is used for scene category recognition. However, the system does not recognize a particular scene or location; it recognizes only a scene category (e.g., junction, corridor, lift lobby).

This approach is similar to our cognitive abilities; we are able to recognize different scene categories (e.g., junction) in a novel place. By combining different scenes categories with their spatial relations, we can create a mental topological representation of an environment. This representation may be used for navigation later. Moreover, thanks to this mental representation, we do not need to remember the visual details for a particular scene instance recognition - as long as a starting location is known (Greene, Baldassano, Esteva,

\section{Algorithm 1. Navigation Based on Pre-defined Environment Topology.}

1 Load topology

2 Initialize current location //starting location needs to be provided

3 Initialize current heading direction //starting orientation needs to be provided

4 Initialize final location

5 Prepare the navigation path

6 repeat

7 Define next node

8 Provide navigation command

9 repeat

10 if walking

$11 \quad$ scene changed $\leftarrow$ is scene changed

$12 \quad$ turn detected $\leftarrow$ is turn detected

13 end

14 until scene changed or turn detected

15 until final node reached 
Beck, \& Fei-Fei, 2016; Greene \& Fei-Fei, 2014). Thanks to the scene category recognition, instead of a precise location recognition, our system does not have to store any particular scene features or any other metrics - we need less storage. Also, the scene category recognition process is faster, especially for large environments, when compared to a particular scene recognition process - current scene features need to be compared against all stored scenes features. The accelerometer is used to detect user movement. If a user is moving - walking forward - the system recognizes scene category and detect turns with the use of gyroscope data. When the user is stationary - not walking forward - the system does not process the video data and does not anticipate any turn.

Algorithm 2 shows how our system works when it creates a topology structure of an environment from collected data (i.e., video and sensor data). During this process, our system detects scene category changes and detects turns. Thanks to this information, the system is able to create a topological map of an environment, which may be used for navigation later.

\section{Algorithm 2. Create Topological Structure of an Environment from Google Glass Data.}

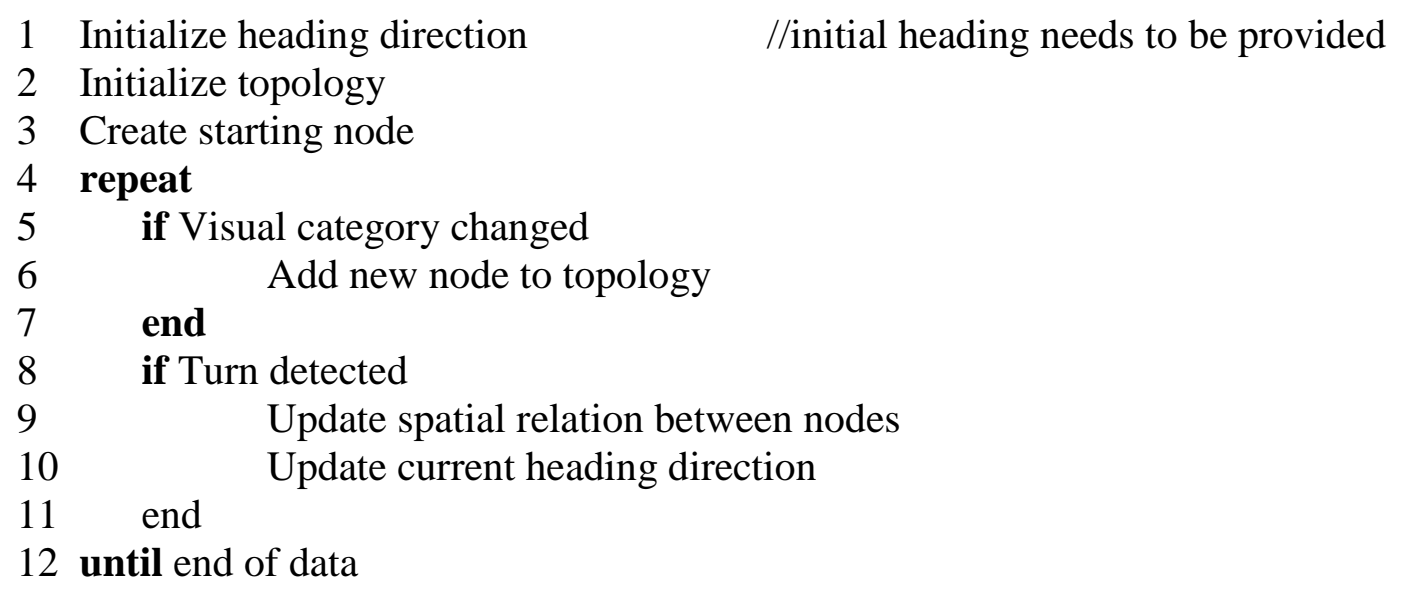




\subsection{Scene Category Recognition using Pre-Trained CNN and SVM}

Visual information is the system's main input. It is a video captured with a wearable camera (e.g., Google Glass). The camera provides visual information from first person view egocentric representation. Video frames, extracted from a video, are next processed by a pretrained Convolution Neural Network $(\mathrm{CNN})$. We have used very deep (i.e., 16 layers) CNN released to the public by the Visual Geometry Group from Oxford University (Simonyan \& Zisserman, 2014). The network output, of the processed video frame, is used as an input to a Support Vector Machine (SVM). This approach is popular among researchers who do not want to (or cannot, due to computational or time requirements) train, or re-train, their own CNN. They can still take advantage of a CNN performance, while using their own output categories (Tang, 2013). The SVM is used for scene categories remapping - the CNN recognizes 1000 categories, while our SVM re-map them to 4 categories. In our current system we used 4 scene categories: junction, corridor, lift lobby, and inside lift. In the future, more categories can be easily added as needed. For the SVM training, we used 4000 video frames (1000 frames per category). During cross-validation (2000 frames for training, 2000 frames for testing), over 100 random splits of the dataset, the average scene categorization error was $5 \%$. As the frames were selected by random splits, it is possible that the same scene was present in both, the training and the testing sets; however, even in this case, a frame in the training set presents a scene from a distinct perspective than a frame in the testing set. Therefore, in our study, we have assumed that a particular scene can be used for both, training and testing of the $\mathrm{CNN}+\mathrm{SVM}$ solution, as long as it is presented from a unique perspective in the training and testing sets. 


\subsection{Step and Turn Detection with Use of Accelerometer and Gyroscope}

We have used an accelerometer and gyroscope information to analyse user's movement. The accelerometer measurements are used to detect steps - check if a user is walking. While the gyroscope measurements are used to detect turns - user's orientation changes.

\subsubsection{Step Detection with Use of Accelerometer Measurements}

We used a step detection process to check if a user is walking. For this purpose, we used an improved method described by Link, Smith, Viol, and Wehrle (2011) and presented in Algorithm 3. The original method measured the linear acceleration towards the ground. From this signal, a step is detected by picking up linear acceleration magnitude that exceeds a certain threshold. Moreover, for the magnitude to be chosen as a step, it must be greater than one of its following data points by a pre-defined amount, while the following data points must lie within a given time interval. When the step is detected, a sleep time starts, during this period no step can be detected. When the dead time finishes, the step detection process starts from the beginning.

Our improvement to the above method adds an additional condition for walk detection. Walk is detected only when at least 3 consecutive steps (i.e., within a given time interval) are made. We added this condition after practical experiments with the original algorithm. The original algorithm was designed for step detection with hand held mobile phone. In this scenario, part of unwanted shakes is suppressed by our natural (often unconscious) ability to stabilize our hand. Therefore, for the accelerometer located in the Google Glass, which, in turn, is located on user's head, some modifications were necessary. The threshold value, a maximum time for 3 consecutive steps, and time after which a next step can be detected were obtained manually, by comparing the ground truth data (videos) with algorithm's predictions and adjusting its parameters. 


\section{Algorithm 3. Walking Detection.}

1 Initialize accelTreshold $:=0.3 \quad / /$ threshold defined as a step

2 Initilize $\Delta \mathrm{T}:=3 \mathrm{sec} \quad / / \max$ time for 3 consecutive steps

3 Initialize sleepTime $:=330 \mathrm{~ms} \quad / /$ time after which next step can be detected

4 noOfSteps $:=0$

5 repeat

6 if (accel $>$ accelTreshold) and noSleepTime

$7 \quad$ step detected

$8 \quad$ stepTimestamp[noOfSteps] $\leftarrow$ current timestamp

9 noOfSteps $=$ noOfSteps +1

10 start sleepTime

11 end

12 if noOfSteps $>2$ and (stepTimestamp[last] - stepTimestamp[first]) $<\Delta$ T

13 walk detected

14 end

15 until end of data

\subsubsection{Turn Detection with Use of Gyroscope Measurements}

We used a turn detection process to check if a user made a turn (i.e., changes walking direction), and to define turning direction. For this purpose, we used a method described by Köping, Ebner, Grzegorzek, and Deinzer (2014) and presented in Algorithm 4. The method uses gyroscope data as a turn detection source. In our system, the Google Glass gyroscope is used. The rotation speed of the Google Glass is measured. When a measurement is bigger, by a certain threshold, than the neighbouring measurements detected, it is denoted as a turn. Also, the duration of this biggest measurement must be longer than a pre-defined time. Similarly to the walking detection, another turn can be detected only after a pre-defined time. 


\section{Algorithm 4. Turn Detection.}

1 Initialize rotationTreshold $:=0.7 / /$ rotation threshold defined as turn

2 Initilize $\Delta \mathrm{T}:=300 \mathrm{~ms} \quad / / \mathrm{min}$ duration of a turn

3 Initialize sleepTime $:=2 \mathrm{~s} \quad / /$ time after which next turn can be detected

4 repeat

5 if (rotation > rotationTreshold) and noSleepTime

$6 \quad$ if duration of the rotation $>\Delta \mathrm{T}$

$7 \quad$ if rotation $>0$

$8 \quad$ left turn detected

$9 \quad$ else $10 \quad$ right turn detected

$11 \quad$ end

12 start sleepTime

13 end

14 end

15 until end of data

Also, as for the step detection algorithm, the rotation threshold, a minimum duration of a turn, and time after which next turn can be detected were obtained manually, by observing the ground truth data (videos) with turn detection algorithm predictions and adjusting those 3 parameters to obtain the best accuracy. A left turn is detected when the measurement value is positive, while a right turn is detected when the value is negative.

For simplicity, we assume that every turn has an angle of $90^{\circ}$. This make it easier to build a topological map of an environment and to provide navigation for pre-mapped environments. When a turn is detected, current user's heading is updated accordingly.

\subsection{Create a Topological Map of an Environment from Google Glass Data}

Our system holds a representation of an environment in a form of a topological map (see bottom-right corner of Figure 27). A topology consists of nodes connected by edges. Each node represents a particular scene category, while an edge denotes the spatial relation between two nodes (e.g., node $\mathrm{Y}$ is north from node $\mathrm{X}$ ). 
Our system is able to build a topological representation of an environment from egocentric video (captured during exploration of a new place) and sensory data (accelerometer and gyroscope) collected together with the video. The system extracts scenes categories encountered along the navigation path and connect them according to the spatial relation between them. Thanks to this process, we obtain a topological representation of the environment, as presented at the bottom-right corner of Figure 27.

Figure 27 presents a sample process of creating a topological structure of an environment from video and gyroscope data. First, a starting node is created. The node represents scene category recognized for a first frame of a video obtained along the exploration of the environment. As we analyse the next frames of the video, our system adds additional nodes to the topology (see Figure 27). Also, thanks to the gyroscope data, spatial relation between two adjacent nodes is included in the topology. 


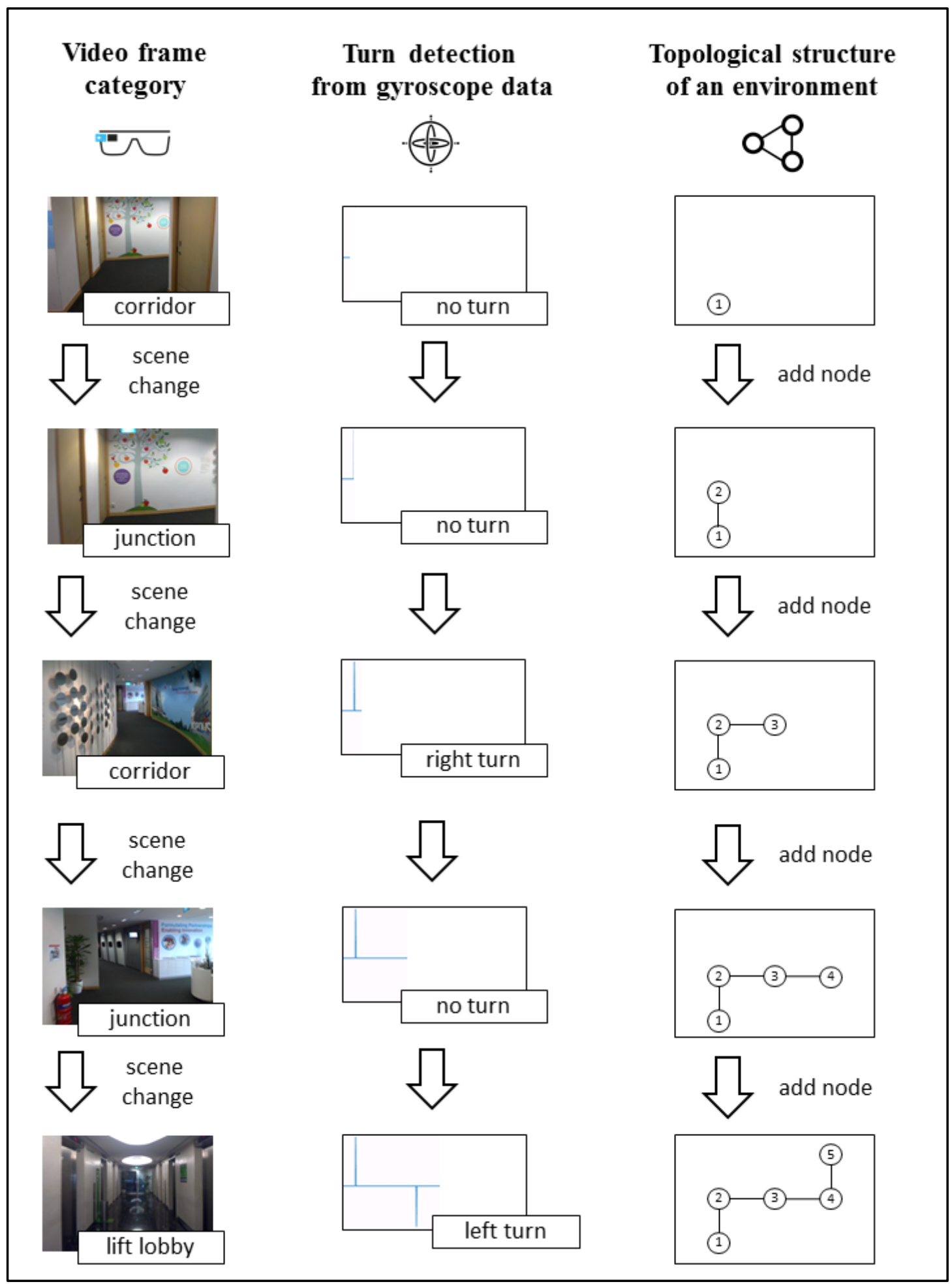

Figure 27. Process of creating a topological structure of an environment with use of video and gyroscope data. First column shows how video frame category changes. Second column presents corresponding turn detections from gyroscope data. Finally, the last column, presents corresponding topology of the environment. 


\subsection{Guidance with Use of Google Glass and a Topological Map of an}

\section{Environment}

The main goal of our system is to provide reliable, fast, and user-friendly guidance through an unfamiliar environment. To achieve that, the system uses a pre-defined topological representation of an environment. Also, the starting location (i.e., starting node) is known before navigation starts. Figure 28 presents a sample guiding process. If a user is moving, the system analyses video frames and gyroscope data. Based on this information, the system is able to predict current user's location and to provide navigation instructions necessary for reaching a final destination.

The current location of a user is computed from previously known position (i.e., previous node) combined with information about scene category changes and a user's movement (a process called path integration (McNaughton et al., 2006)). As presented in Figure 28, if a new scene category matches scene category associated with a next node expected along the navigation path, the current location is updated. Also, gyroscope data is used to detect user's turns, and to update user's orientation within the topological structure. The process of detecting scene categories changes and detecting turns, continues until a final location is reached. 


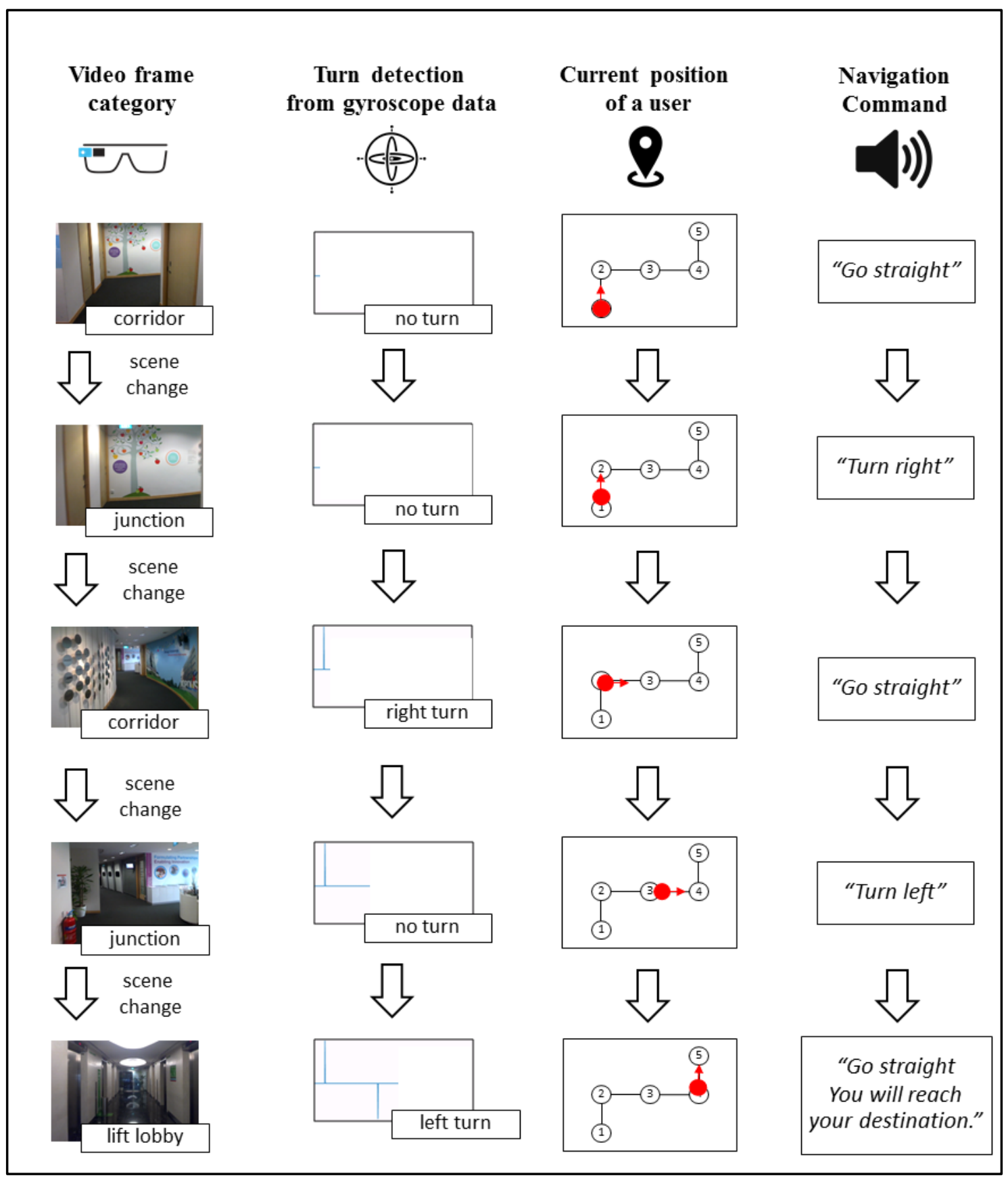

Figure 28. Navigation process with use of pre-created topological structure of an environment. Starting position is provided. Video and gyroscope data is used to define following positions within the topology. First column shows how video frame category changes. Second column presents corresponding turn detections from gyroscope data.

Third column, presents corresponding topology of the environment together with current user's location. Finally, the last column, presents navigation commands provided to a user. 


\subsection{Conclusions}

In this chapter, we presented a design and proof-of-concept implementation of a novel, cognitive-inspired, indoor-navigation system. First, the system is able to automatically extract topological representation by exploring the environment. In this process, our system uses video and sensors data collected in first person view perspective, by a user who explored the environment. During operation, the system provides navigation guidance through its mapped representation of the environments. This kind of navigation assistant is especially important for people with cognitive impairment in navigation abilities, e.g. dementia, inability to localize oneself in the environment (Corkin, 1984), or with difficulties in long-term planning (Luria, 1966).

Moreover, topological representations created with the use of our system, can be transferred to and used by other systems. Also, the topological representations of an environment can be created manually in a simple and intuitive manner (e.g., by shopping mall owner) and used by our system for guidance. Finally, our system does not require any additional infrastructure for localization (e.g., Wi-Fi routers, Bluetooth beacons, etc); hence it is as inexpensive as other localization systems that utilize image recognition. However, unlike those systems, our solution does not require storing extensive data necessary for image recognition (e.g., image features), as it utilizes a convolutional neural network for scene category recognition. Moreover, information about previous scenes categories and movement data, current scene, movement, and expected scene category, allows our system to use this congregated data for accurate and efficient guidance. In general, our system is designed to be cheaper and more satisfactory than most of the currently available solutions, while maintaining similar efficiency as those solutions.

In the future, our system can be improved by making the turn detection method more precise - detect the actual turning angle. We may also improve the walking detection method 
Cognitive Navigation System

with a step length added, which would allow the creation of a topological map with distance information between two nodes. 


\section{Conclusions and Future Perspectives}

In this thesis, we have reviewed studies on visual perception and memory; we summarized how cognitive neurobiology and psychology define different human memory systems. We also presented our review on human navigation. We presented the necessary components for human navigation. Next, we presented our studies on: 1) how different types of navigation assistance affect our visual memory of scenes encountered during exploration of a novel environment, 2) how backtracking and repetition priming can improve our navigation abilities, 3) how different Google Glass interaction modalities affect our navigation abilities and subjective experience of the navigation system. Finally, we proposed, and showed a novel, cognitive-inspired navigation system. The system mimics our cognitive representation and inference in navigation; hence, it is efficient and human-like in mapping vast indoor environments and in providing user-centered guidance.

Our first experiment (see Sections 3.1 to 3.3) focused on better understanding scene memorability during guided indoor navigation. Motivated by studies on image memorability and human navigation, we designed an experiment which provides valuable and interesting findings on these research areas. We recruited twenty participants, who were guided by three types of navigation assistance - a 2D map, a wearable virtual navigator, and a human guide through an unfamiliar indoor environment. To the best of our knowledge, this is the first experiment that analyses the influence of different indoor navigation assistance, and associated cognitive loads, on scene memorability. Human performance consistency (Spearman's rank correlation $(\rho)=0.75)$ shows that most of the participants remembered roughly the same visual scenes. This supports earlier works on image memorability (Isola, Parikh, et al., 2011). In addition, the memorability scores reveal that the participants remembered the same visual scenes while guided by different types of navigation assistance, and exposed to corresponding cognitive loads. These results may indicate that spatial 
working memory, required for navigation, may be dissociated with visual memory. In addition, since wearable assistance neither impact scene memorability nor navigation performance, there is no need to change design principles of indoor navigation signs.

Moreover, our results show that the participants tend to remember scenes with navigation information, rather than scenes that do not contain such information. This finding might explain why lift lobbies scenes - which contain many navigation prompts - were more memorable than scenes at different locations.

Our second experiment (see Sections 3.4 to 3.6) aimed to check if (and if yes, then how) backtracking or repetitive priming is able to enhance our memory of visited scenes during navigation. For this study, we recruited 20 participants. As expected, after backtracking, the participants correctly recognized more encountered scenes. Their visual memory of encountered scenes was enhanced as their attention was driven by the backtracking task. Also, while searching for landmarks, the participants probably remembered other less crucial but still memorable scenes.

However, the repetition priming does not further enhance visual memory of our participants. The participants from the primed group remembered similar number of scenes when compared to the participants who were not exposed to the priming. This may be explained by the following reasons. Firstly, our attention is strongly guided by a top-down attention (i.e., given task) independently of priming (Leonard \& Egeth, 2008). It is highly possible that the participants focused only on the backtracking task and were insensitive to the priming effect. Also, the cognitive load - caused by the backtracking task - might be too high, and thwarts further improvement of the participants' visual memory.

Next, our research focused on evaluating Google Glass (GG) as indoor navigation assistance (see Chapter 4, Do Navigation Assistance Interaction Modalities Affect Spatial Cognition). Also, we investigated different Google Glass interaction modalities: voice, 
display, voice+display. We compared them against Human Guide (HG). Several objective measurements were adopted: navigation and backtracking performance, correct backtracking decisions, and visual memory. Also, we measured subjective user experience with the use of questionnaires. We recruited sixty participants, who were guided by GG and HG through an unfamiliar indoor environment. For results analysis, we used data collected from fifty-one participants. To the best of our knowledge, this was the first experiment that investigated GG's impact on users' spatial cognition and visual memory, compared its performance (to that of $\mathrm{HG})$, and studied subjective user experience.

We showed that navigation assistance based on GG with the voice+display modality can be as effective as HG during guided navigation task. However, using the voice+display modality negatively affects participants' spatial cognition - backtracking performance. Also, we found that none of the navigation systems (i.e., GG and HG) affected participants' visual memory, nor do the presentation modalities. All participants remembered similar number of scenes encountered along the navigation route.

Finally, this thesis (see Chapter 5, Towards a Novel Cognitive-Inspired Indoor Navigation System) presents a novel, wearable, cognitive-inspired navigation system. The system is able to extract topological representation of an explored environment automatically. Based on a pre-defined topology, the system can provide efficient guidance. Also, a topological representation of an environment may be transferred and used by other systems. Our system provides a foundation for a wearable, cognitive-based, and efficient navigation solutions that may be deployed in several scenarios (e.g., shopping malls, airports, hospitals, offices, etc.).

In general, advances in wearable cameras (e.g., Google Glass) make it possible to develop accurate, trustworthy, personal navigation assistance. In addition, with welldesigned, clear, friendly interface, this kind of systems may be more desirable and preferred 
over other kinds of navigation assistance (e.g. digital maps). This kind of navigation assistance is especially important for people with cognitive impairment in navigation abilities, like: dementia, inability to localize oneself in the environment (Corkin, 1984). Our research, summarized in this thesis, leads to useful insights on human navigation abilities, especially on how different types of navigation assistance, different interaction modalities, and external stimuli (i.e., priming) affect these abilities; also, our work provides implications for the design of modern, user-centred navigation systems. By examining how these devices impact human visual memory, it becomes possible to adapt these systems to user's needs - as we demonstrated with a novel, cognitive-inspired, navigation system. 


\section{References}

Abu-Ghazzeh, T. M. (1996). Movement and wayfinding in the King Saud University built environment: A look at freshman orientation and environmental information. Journal of Environmental Psychology, 16(4), 303-318.

Allen, G. L. (1999). Spatial abilities, cognitive maps, and wayfinding. Wayfinding behavior: Cognitive mapping and other spatial processes, 46-80.

Anderson, J. R. (2000). Learning and memory (Vol. 86): John Wiley New York.

Anderson, J. R. (2007). How can the human mind occur in the physical universe? : Oxford University Press.

Anderson, J. R., Bothell, D., Byrne, M. D., Douglass, S., Lebiere, C., \& Qin, Y. (2004). An integrated theory of the mind. Psychological Review, 111(4), 1036.

Arikawa, M., Konomi, S. i., \& Ohnishi, K. (2007). NAVITIME: Supporting pedestrian navigation in the real world. IEEE Pervasive Computing(3), 21-29.

Arning, K., Ziefle, M., Li, M., \& Kobbelt, L. (2012). Insights into user experiences and acceptance of mobile indoor navigation devices. Proceedings of the $11^{\text {th }}$ International Conference on Mobile and Ubiquitous Multimedia, Ulm, Germany.

Atkinson, R. C., \& Shiffrin, R. M. (1968). Human memory: A proposed system and its control processes. The psychology of learning and motivation, 2, 89-195.

Baddeley, A. (2000). The episodic buffer: a new component of working memory? Trends in cognitive sciences, 4(11), 417-423.

Baddeley, A. D., \& Hitch, G. (1974). Working memory. Psychology of learning and motivation, 8, 47-89.

Böök, A., \& Gärling, T. (1981). Maintenance of orientation during locomotion in unfamiliar environments. Journal of Experimental Psychology: Human Perception and Performance, 7(5), 995. 
Borji, A., \& Itti, L. (2013). State-of-the-art in visual attention modeling. IEEE Transactions on Pattern Analysis and Machine Intelligence, 35(1), 185-207.

Brady, T. F., Konkle, T., \& Alvarez, G. A. (2011). A review of visual memory capacity: Beyond individual items and toward structured representations. Journal of Vision, $11(5), 1-34$

Brewer, W. F., \& Treyens, J. C. (1981). Role of schemata in memory for places. Cognitive Psychology, 13(2), 207-230.

Brown, M., \& Pinchin, J. (2013). Exploring Human Factors in Indoor Navigation. Proceedings of the European Navigation Conference.

Burgess, N. (2006). Spatial memory: how egocentric and allocentric combine. Trends in cognitive sciences, 10(12), 551-557.

Chang, Y.-J., Tsai, S.-K., Chang, Y.-S., \& Wang, T.-Y. (2007). A novel wayfinding system based on geo-coded qr codes for individuals with cognitive impairments. Proceedings of the $9^{\text {th }}$ international ACM SIGACCESS conference on Computers and accessibility, Tempe, Arizona, USA.

Chang, Y.-J., Tsai, S.-K., \& Wang, T.-Y. (2008). A context aware handheld wayfinding system for individuals with cognitive impairments. Proceedings of the $10^{\text {th }}$ international ACM SIGACCESS conference on Computers and accessibility.

Chumkamon, S., Tuvaphanthaphiphat, P., \& Keeratiwintakorn, P. (2008). A blind navigation system using RFID for indoor environments. Proceedings of the $5^{\text {th }}$ International Conference on Electrical Engineering/Electronics, Computer, Telecommunications and Information Technology, 2008. ECTI-CON 2008.

Connor, C. E., Egeth, H. E., \& Yantis, S. (2004). Visual attention: bottom-up versus topdown. Current Biology, 14(19), R850-R852. 
Corkin, S. (1984). Lasting consequences of bilateral medial temporal lobectomy: Clinical course and experimental findings in HM. Proceedings of the Seminars in Neurology.

Costa, T., Epps, S. R., Husson, T., Ask, J. A., Sheldon, P., Doty, C. A., \& Vokshi, A. (2013). Indoor venues are the next frontier for location-based services. http://www.forbes.com/sites/forrester/2013/01/23/indoor-venuesare-the-next-frontierfor-location-based-services/.

Cutting, J. E. (1996). Wayfinding from multiple sources of local information in retinal flow. Journal of Experimental Psychology: Human Perception and Performance, 22(5), 1299-1313.

D'Atri, E., Medaglia, C. M., Serbanati, A., Ceipidor, U. B., Panizzi, E., \& D'Atri, A. (2007). A system to aid blind people in the mobility: A usability test and its results. Proceedings of the $2^{\text {nd }}$ International Conference on the Systems, 2007. ICONS'07.

Dalal, N., \& Triggs, B. (2005). Histograms of oriented gradients for human detection. Proceedings of the IEEE Computer Society Conference on Computer Vision and Pattern Recognition (CVPR).

Dieberger, A., \& Frank, A. U. (1998). A city metaphor to support navigation in complex information spaces. Journal of Visual Languages \& Computing, 9(6), 597-622.

Ding, B., Yuan, H., Jiang, L., \& Zang, X. (2007). The research on blind navigation system based on RFID. Proceedings of the International Conference on Wireless Communications, Networking and Mobile Computing, 2007. WiCom 2007.

Doeller, C. F., Barry, C., \& Burgess, N. (2012). From cells to systems: grids and boundaries in spatial memory. Neuroscientist, 18(6), 556-566.

Duch, W., Oentaryo, R. J., \& Pasquier, M. (2008). Cognitive Architectures: Where do we go from here? Frontiers in Artificial Intelligence and Applications, 171, 122. 
Eichenbaum, H., Yonelinas, A., \& Ranganath, C. (2007). The medial temporal lobe and recognition memory. Annual Review of Neuroscience, 30, 123.

Fairchild, M. D. (2013). Color appearance models: John Wiley \& Sons.

Fernández-Torné, A., \& Matamala, A. (2015). Text-to-speech vs. human voiced audio descriptions: a reception study in films dubbed into Catalan. The Journal of Specialised Translation, 24, 61-88.

Foo, P., Duchon, A., Warren Jr, W. H., \& Tarr, M. J. (2007). Humans do not switch between path knowledge and landmarks when learning a new environment. Psychological Research, 71(3), 240-251.

Foo, P., Warren, W. H., Duchon, A., \& Tarr, M. J. (2005). Do humans integrate routes into a cognitive map? Map-versus landmark-based navigation of novel shortcuts. Journal of Experimental Psychology: Learning, Memory, and Cognition, 31(2), 195.

Froehlich, P., Baillie, L., \& Simon, R. (2008). FEATURE: Realizing the vision of mobile spatial interaction. interactions, 15(1), 15-18. doi: 10.1145/1330526.1330534

Fyhn, M., Hafting, T., Treves, A., Moser, M.-B., \& Moser, E. I. (2007). Hippocampal remapping and grid realignment in entorhinal cortex. Nature, 446(7132), 190-194.

Gale, N., Golledge, R. G., Pellegrino, J. W., \& Doherty, S. (1990). The acquisition and integration of route knowledge in an unfamiliar neighborhood. Journal of Environmental Psychology, 10(1), 3-25.

Giannopoulos, I., Kiefer, P., Raubal, M., Richter, K.-F., \& Thrash, T. (2014). Wayfinding Decision Situations: A Conceptual Model and Evaluation. In M. Duckham, E. Pebesma, K. Stewart \& A. Frank (Eds.), Geographic Information Science (Vol. 8728, pp. 221-234): Springer International Publishing.

Gibson, J. (1986). The ecological approach to visual perception: Routledge. 
Goertzel, B., Lian, R., Arel, I., de Garis, H., \& Chen, S. (2010). A world survey of artificial brain projects, Part II: Biologically inspired cognitive architectures. Neurocomputing, 74(1), 30-49.

Goodale, M. A., \& Milner, A. D. (1992). Separate visual pathways for perception and action. Trends in neurosciences, 15(1), 20-25.

Greene, M. R., Baldassano, C., Esteva, A., Beck, D. M., \& Fei-Fei, L. (2016). Visual scenes are categorized by function. Journal of Experimental Psychology: General, 145(1), 82.

Greene, M. R., \& Fei-Fei, L. (2014). Visual categorization is automatic and obligatory: Evidence from Stroop-like paradigm. Journal of Vision, 14(1), 14-14.

Grierson, L. E. M., Zelek, J., \& Carnahan, H. (2009). The Application of a Tactile Wayfinding Belt to Facilitate Navigation in Older Persons. Ageing International, 34(4), 203-215. doi: 10.1007/s12126-009-9039-2

Grossberg, S. (2012). Adaptive Resonance Theory: How a brain learns to consciously attend, learn, and recognize a changing world. Neural Networks.

Hafting, T., Fyhn, M., Molden, S., Moser, M. B., \& Moser, E. I. (2005). Microstructure of a spatial map in the entorhinal cortex. Nature, 436(7052), 801-806. doi: 10.1038/nature03721

Harle, R. (2013). A Survey of Indoor Inertial Positioning Systems for Pedestrians. IEEE Communications Surveys \& Tutorials, 15(3), 1281-1293. doi: 10.1109/SURV.2012.121912.00075

Harrison, A. M., \& Schunn, C. D. (2003). ACT-R/S: Look Ma, no "cognitive-map". Proceedings of the International Conference on Cognitive Modeling, Bamberg, Germany: Universitas-Verlag Bamberg. 
Hegarty, M., Montello, D. R., Richardson, A. E., Ishikawa, T., \& Lovelace, K. (2006). Spatial abilities at different scales: Individual differences in aptitude-test performance and spatial-layout learning. Intelligence, 34(2), 151-176.

Herd, S. A., \& O’Reilly, R. C. (2005). Serial visual search from a parallel model. Vision research, 45(24), 2987-2992.

Hightower, J., \& Borriello, G. (2001). Location systems for ubiquitous computing. Computer, 34(8), 57-66.

Hile, H., Vedantham, R., Cuellar, G., Liu, A., Gelfand, N., Grzeszczuk, R., \& Borriello, G. (2008). Landmark-based pedestrian navigation from collections of geotagged photos. Proceedings of the $7^{\text {th }}$ International Conference on Mobile and Ubiquitous Multimedia.

Hollingworth, A., \& Henderson, J. M. (1998). Does consistent scene context facilitate object perception? Journal of Experimental Psychology: General, 127(4), 398-415.

Huang, H., Schmidt, M., \& Gartner, G. (2012). Spatial Knowledge Acquisition with Mobile Maps, Augmented Reality and Voice in the Context of GPS-based Pedestrian Navigation: Results from a Field Test. Cartography and Geographic Information Science, 39(2), 107-116. doi: 10.1559/15230406392107

Huang, L., Holcombe, A. O., \& Pashler, H. (2004). Repetition priming in visual search: Episodic retrieval, not feature priming. Memory \& Cognition, 32(1), 12-20.

Hub, A., Diepstraten, J., \& Ertl, T. (2004). Design and development of an indoor navigation and object identification system for the blind. Proceedings of the ACM Sigaccess Accessibility and Computing.

Isola, P., Parikh, D., Torralba, A., \& Oliva, A. (2011). Understanding the intrinsic memorability of images. Proceedings of the Advances in Neural Information Processing Systems. 
Isola, P., Xiao, J., Torralba, A., \& Oliva, A. (2011). What makes an image memorable? Proceedings of the IEEE Conference on Computer Vision and Pattern Recognition (CVPR).

Itti, L., Koch, C., \& Niebur, E. (1998). A model of saliency-based visual attention for rapid scene analysis. IEEE Transactions on Pattern Analysis and Machine Intelligence, 20(11), 1254-1259.

James, W. (1890). 1950. The principles of psychology (pp. 403-404): New York: Dover Publications.

Jilk, D. J., Lebiere, C., O’Reilly, R. C., \& Anderson, J. R. (2008). SAL: An explicitly pluralistic cognitive architecture. Journal of Experimental and Theoretical Artificial Intelligence, 20(3), 197-218.

Joseph, S. L., Zhang, X., Dryanovski, I., Xiao, J., Yi, C., \& Tian, Y. (2013). Semantic indoor navigation with a blind-user oriented augmented reality. Proceedings of the IEEE International Conference on Systems, Man, and Cybernetics (SMC), 2013.

Kalkusch, M., Lidy, H., Knapp, M., Reitmay, G., Kaufmann, H., \& Schmalstieg, D. (2002). Structured visual markers for indoor pathfinding. Proceedings of the $1^{\text {st }}$ IEEE International Workshop on Augmented Reality Toolkit.

Kallie, C. S., Schrater, P. R., \& Legge, G. E. (2007). Variability in stepping direction explains the veering behavior of blind walkers. Journal of Experimental Psychology: Human Perception and Performance, 33(1), 183.

Kearns, M. J., Warren, W. H., Duchon, A. P., \& Tarr, M. J. (2002). Path integration from optic flow and body senses in a homing task. Perception, 31, 349-374.

Kennedy, W. G., \& Trafton, J. G. (2007). Long-term symbolic learning in soar and act-r: DTIC Document. 
Khosla, A., Xiao, J., Isola, P., Torralba, A., \& Oliva, A. (2012). Image memorability and visual inception. Proceedings of the SIGGRAPH Asia.

Khosla, A., Xiao, J., Torralba, A., \& Oliva, A. (2012). Memorability of image regions. Proceedings of the Advances in Neural Information Processing Systems.

Klatzky, R. L. (1998). Allocentric and Egocentric Spatial Representations: Definitions, Distinctions, and Interconnections. Proceedings of the Spatial Cognition, An Interdisciplinary Approach to Representing and Processing Spatial Knowledge.

Koch, K., McLean, J., Segev, R., Freed, M. A., Berry II, M. J., Balasubramanian, V., \& Sterling, P. (2006). How Much the Eye Tells the Brain. Current Biology, 16(14), $1428-1434$.

Krüger, A., Aslan, I., \& Zimmer, H. (2004). The effects of mobile pedestrian navigation systems on the concurrent acquisition of route and survey knowledge. Mobile HumanComputer Interaction-MobileHCI 2004 (pp. 446-450): Springer.

Lahey, B. B., McNealy, K., Knodt, A., Zald, D. H., Sporns, O., Manuck, S. B., Hariri, A. R. (2012). Using confirmatory factor analysis to measure contemporaneous activation of defined neuronal networks in functional magnetic resonance imaging. Neuroimage, 60(4), 1982-1991. doi: 10.1016/j.neuroimage.2012.02.002

Laird, J. E. (2012). The Soar cognitive architecture: MIT Press.

LaMarca, A., Chawathe, Y., Consolvo, S., Hightower, J., Smith, I., Scott, J., Potter, F. (2005). Place lab: Device positioning using radio beacons in the wild. Proceedings of the International Conference on Pervasive Computing.

Lathrop, S. D., Wintermute, S., \& Laird, J. E. (2011). Exploring the functional advantages of spatial and visual cognition from an architectural perspective. Topics in Cognitive Science, 3(4), 796-818.

Lee, D. N. (1974). Visual information during locomotion. Perception, 250-267. 
Leonard, C. J., \& Egeth, H. E. (2008). Attentional guidance in singleton search: An examination of top-down, bottom-up, and intertrial factors. Visual Cognition, 16(8), 1078-1091.

Li, H., Zhang, Y., Wu, C., \& Mei, D. (2016). Effects of Field Dependence-Independence and Frame of Reference on Navigation Performance Using Multi-dimensional Electronic Maps. Personality and Individual Differences, 97, 289-299. doi: http://dx.doi.org/10.1016/j.paid.2016.03.078.

Li, L., Wang, G. S., Goh, W., Lim, J.-H., \& Tan, C. (2013). A Wearable Cognitive Vision System for Navigation Assistance in Indoor Environment. Proceedings of the $26^{\text {th }}$ Annual Conference on Neural Information Processing Systems (NIPS), Lake Tahoe.

Li, L., Xu, Q., Chandrasekhar, V., Lim, J. H., Tan, C., \& Mukawa, M. A. (2016). A Wearable Virtual Usher for Vision-Based Cognitive Indoor Navigation. IEEE Transactions on Cybernetics, PP(99), 1-14. doi: 10.1109/TCYB.2016.2530407

Link, J. A. B., Smith, P., Viol, N., \& Wehrle, K. (2011). Footpath: Accurate map-based indoor navigation using smartphones. Proceedings of the International Conference on Indoor Positioning and Indoor Navigation (IPIN), 2011.

Liu, A. L., Hile, H., Borriello, G., Brown, P. A., Harniss, M., Kautz, H., \& Johnson, K. (2009). Customizing directions in an automated wayfinding system for individuals with cognitive impairment. Proceedings of the $11^{\text {th }}$ International ACM SIGACCESS Conference on Computers and Accessibility, Pittsburgh, PA, USA.

Loomis, J. M., Marston, J. R., Golledge, R. G., \& Klatzky, R. L. (2005). Personal guidance system for people with visual impairment: A comparison of spatial displays for route guidance. Journal of visual impairment \& blindness, 99(4), 219.

Lowe, D. G. (2004). Distinctive image features from scale-invariant keypoints. International journal of computer vision, 60(2), 91-110. 
Luck, S. J., \& Hollingworth, A. (2008). Visual memory: Oxford University Press, USA.

Luria, A. R. (1966). Higher cortical functions in man. Basic Books.

Lynch, K. (1960). The image of the city (Vol. 11): MIT press.

Macmillan, N. A., \& Creelman, C. D. (2004). Detection theory: A user's guide: Psychology press.

Maguire, E. A., Burgess, N., Donnett, J. G., Frackowiak, R. S., Frith, C. D., \& O'Keefe, J. (1998). Knowing where and getting there: a human navigation network. Science, 280(5365), 921-924.

Maljkovic, V., \& Nakayama, K. (1994). Priming of pop-out: I. Role of features. Memory \& Cognition, 22(6), 657-672.

Mancas, M., \& Le Meur, O. (2013). Memorability of Natural Scenes: The Role of Attention. Proceedings of the International Conference on Image Processing.

May, A. J., Ross, T., Bayer, S. H., \& Tarkiainen, M. J. (2003). Pedestrian navigation aids: information requirements and design implications. Personal and Ubiquitous Computing, 7(6), 331-338.

McNaughton, B. L., Battaglia, F. P., Jensen, O., Moser, E. I., \& Moser, M.-B. (2006). Path integration and the neural basis of the'cognitive map'. Nature Reviews Neuroscience, 7(8), 663-678.

Millonig, A., \& Schechtner, K. (2007). Developing Landmark-Based Pedestrian-Navigation Systems. IEEE Transactions on Intelligent Transportation Systems, 8(1), 43-49. doi: 10.1109/TITS.2006.889439

Misra, P., \& Enge, P. (2006). Global Positioning System: Signals, Measurements and Performance Second Edition. Massachusetts: Ganga-Jamuna Press. 
Möller, A., Kranz, M., Diewald, S., Roalter, L., Huitl, R., Stockinger, T., Lindemann, P. A. (2014). Experimental evaluation of user interfaces for visual indoor navigation. Proceedings of the SIGCHI Conference on Human Factors in Computing Systems.

Moller, S., Engelbrecht, K. P., Kuhnel, C., Wechsung, I., \& Weiss, B. (2009, 29-31 July 2009). A taxonomy of quality of service and Quality of Experience of multimodal human-machine interaction. Proceedings of the International Workshop on Quality of Multimedia Experience, 2009. QoMEx 2009.

Montello, D. R. (2005). Navigation. The Cambridge handbook of visuospatial thinking, 18, 257-294.

Moscovitch, M., Nadel, L., Winocur, G., Gilboa, A., \& Rosenbaum, R. S. (2006). The cognitive neuroscience of remote episodic, semantic and spatial memory. Current Opinion in Neurobiology, 16(2), 179-190.

Mukawa, M., Tan, C., Lim, J.-H., Xu, Q., \& Li, L. (2015). The effect of repetition priming on spatial memory during backtracking in a novel environment. Cognitive processing, 14. doi: 10.1007/s10339-015-0668-y

Mukawa, M., Tan, C., Lim, J.-H., Xu, Q., \& Li, L. (2017). The effect of different types of navigation assistance on indoor scene memorability. Behaviour \& Information Technology, 1-16. doi: 10.1080/0144929X.2017.1309458

Müller, M., \& Wehner, R. (1988). Path integration in desert ants, Cataglyphis fortis. Proceedings of the National Academy of Sciences, 85(14), 5287-5290.

Münzer, S., Zimmer, H. D., Schwalm, M., Baus, J., \& Aslan, I. (2006). Computer-assisted navigation and the acquisition of route and survey knowledge. Journal of Environmental Psychology, 26(4), 300-308.

Murakoshi, S., \& Kawai, M. (2000). Use of knowledge and heuristics for wayfinding in an artificial environment. Environment and behavior, 32(6), 756-774. 
Nestor, A., \& Kokinov, B. (2004). Towards active vision in the dual cognitive architecture. International Journal on Information Theories and Applications, 11.

Newell, A. (1994). Unified theories of cognition (Vol. 187): Harvard University Press.

Nurmi, P., Salovaara, A., Bhattacharya, S., Pulkkinen, T., \& Kahl, G. (2011). Influence of landmark-based navigation instructions on user attention in indoor smart spaces. Proceedings of the $16^{\text {th }}$ International Conference on Intelligent User Interfaces.

Nuxoll, A. M., \& Laird, J. E. (2007). Extending cognitive architecture with episodic memory. Proceedings of the National Conference on Artificial Intelligence.

O'Keefe, J., \& Dostrovsky, J. (1971). The hippocampus as a spatial map: Preliminary evidence from unit activity in the freely-moving rat. Brain research.

O'Reilly, R. C. (1996). Biologically plausible error-driven learning using local activation differences: The generalized recirculation algorithm. Neural computation, 8(5), 895938.

Ohm, C., Müller, M., \& Ludwig, B. (2016). Evaluating indoor pedestrian navigation interfaces using mobile eye tracking. Spatial Cognition \& Computation, 1-32. doi: $10.1080 / 13875868.2016 .1219913$

Oliva, A., \& Torralba, A. (2006). Building the gist of a scene: The role of global image features in recognition. Progress in brain research, 155, 23-36.

Pahlavan, K., Li, X., \& Mäkelä, J.-P. (2002). Indoor geolocation science and technology. Communications Magazine, IEEE, 40(2), 112-118.

Pashler, H. E. (1999). Attention and Memory The psychology of attention (pp. 319-356): MIT press.

Pfeiffer, B. E., \& Foster, D. J. (2013). Hippocampal place-cell sequences depict future paths to remembered goals. Nature, 497(7447), 74-79. 
Pisoni, D. B. (1997). Perception of synthetic speech Progress in speech synthesis (pp. 541560): Springer.

Posner, M. I. (1980). Orienting of attention. Quarterly journal of experimental psychology, $32(1), 3-25$.

Presson, C. C., \& Montello, D. R. (1988). Points of reference in spatial cognition: Stalking the elusive landmark. British Journal of Developmental Psychology, 6(4), 378-381.

Rajamäki, J., Viinikainen, P., Tuomisto, J., Sederholm, T., \& Säämänen, M. (2007). LaureaPOP indoor navigation service for the visually impaired in a WLAN environment. Proceedings of the $6^{\text {th }}$ WSEAS International Conference on Electronics, Hardware, Wireless and Optical Communications.

Rau, P., \& Wang, Y. (2003). A Study of Navigation Support Tools for Mobile Devices. Proc. HCI Int., Crete, Greece, June, 22-27.

Rehman, U., \& Cao, S. (2015, 9-12 Oct. 2015). Augmented Reality-Based Indoor Navigation Using Google Glass as a Wearable Head-Mounted Display. Proceedings of the IEEE International Conference on Systems, Man, and Cybernetics (SMC), 2015.

Rehman, U., \& Cao, S. (2015). Experimental Evaluation of Indoor Navigation Devices. Proceedings of the the Human Factors and Ergonomics Society Annual Meeting.

Richardson, A. E., Montello, D. R., \& Hegarty, M. (1999). Spatial knowledge acquisition from maps and from navigation in real and virtual environments. Memory \& Cognition, 27(4), 741-750.

Riecke, B. E., van Veen, H. A., \& Bülthoff, H. H. (2002). Visual homing is possible without landmarks: A path integration study in virtual reality. Presence: Teleoperators and Virtual Environments, 11(5), 443-473.

Rogers, E. M. (2010). Diffusion of innovations: Simon and Schuster. 
Ross, D. A., \& Blasch, B. B. (2000). Wearable interfaces for orientation and wayfinding. Proceedings of the the $4^{\text {th }}$ International ACM Conference on Assistive Technologies.

Rugg, M. D., \& Vilberg, K. L. (2012). Brain networks underlying episodic memory retrieval. Current Opinion in Neurobiology.

Samsonovich, A. V. (2010). Toward a Unified Catalog of Implemented Cognitive Architectures. Proceedings of the BICA Annual Meeting.

Serra, A., Carboni, D., \& Marotto, V. (2010). Indoor pedestrian navigation system using a modern smartphone. Proceedings of the $12^{\text {th }}$ International Conference on Human Computer Interaction with Mobile Devices and Services.

Shechtman, E., \& Irani, M. (2007). Matching local self-similarities across images and videos. Proceedings of the IEEE Conference on Computer Vision and Pattern Recognition (CVPR).

Simonyan, K., \& Zisserman, A. (2014). Very deep convolutional networks for large-scale image recognition. arXiv preprint arXiv:1409.1556.

Souman, J. L., Frissen, I., Sreenivasa, M. N., \& Ernst, M. O. (2009). Walking straight into circles. Current Biology, 19(18), 1538-1542.

Srikulwong, M., \& O’Neill, E. (2010). A comparison of two wearable tactile interfaces with a complementary display in two orientations. Proceedings of the International Workshop on Haptic and Audio Interaction Design.

Stackman, R. W., \& Taube, J. S. (1997). Firing properties of head direction cells in the rat anterior thalamic nucleus: dependence on vestibular input. The Journal of neuroscience, 17(11), 4349-4358.

Steinbach, E., Schroth, G., Abu-Alqumsan, M., Huitl, R., Al-Nuaimi, A., \& Schweiger, F. (2017). Visual localization method: Google Patents. 
Sweller, J., Van Merrienboer, J. J., \& Paas, F. G. (1998). Cognitive architecture and instructional design. Educational psychology review, 10(3), 251-296.

Tang, Y. (2013). Deep learning using linear support vector machines. arXiv preprint arXiv:1306.0239.

Taube, J. S. (1995). Head Direction Cells Recorded In The Anterior Thalamic Nuclei Of Freely Moving Rats. Journal of Neuroscience, 15(1), 70-86.

Torres-Méndez, L. A., \& Cervantes-Jacobo, R. (2012). Learning Cognitive Human Navigation Behaviors for Indoor Mobile Robot Navigation. Proceedings of the International Conference on Advanced Cognitive Technologies and Applications.

Tulving, E. (1972). Episodic and semantic memory 1. Organization of Memory. London: Academic, 381(4), 382-404.

Tulving, E. (2002). Episodic memory: From mind to brain. Annual review of psychology, $53(1), 1-25$.

Tulving, E., \& Craik, F. I. (2000). The Oxford handbook of memory: Oxford University Press. van Schaik, P., Mayouf, M., \& Aranyi, G. (2015). 3-D route-planning support for navigation in a complex indoor environment. Behaviour \& Information Technology, 34(7), 713724. doi: 10.1080/0144929X.2015.1004649

Wang, H.-C., Lu, S., Lim, J.-H., \& Pomplun, M. (2012). Visual Attention is Attracted by Text Features Even in Scenes without Text. Proceedings of the $34^{\text {th }}$ Annual Meeting of the Cognitive Science Society (CogSci 2012), Sapporo, Japan.

Wang, H.-C., \& Pomplun, M. (2012). The attraction of visual attention to texts in real-world scenes. Journal of Vision, 12(6).

Wang, R. F., \& Spelke, E. S. (2000). Updating egocentric representations in human navigation. Cognition, 77(3), 215-250. 
Warrington, E. K., \& Weiskrantz, L. (1974). The effect of prior learning on subsequent retention in amnesic patients. Neuropsychologia, 12(4), 419-428.

Wiener, J. M., Büchner, S. J., \& Hölscher, C. (2009). Taxonomy of human wayfinding tasks: A knowledge-based approach. Spatial Cognition \& Computation, 9(2), 152-165.

Willis, K. S., Hölscher, C., Wilbertz, G., \& Li, C. (2009). A comparison of spatial knowledge acquisition with maps and mobile maps. Computers, Environment and Urban Systems, $33(2), 100-110$.

Winkelholz, C., \& Schlick, C. M. (2007). Modeling human spatial memory within a symbolic architecture of cognition. In T. Barkowsky, M. Knauff, G. Ligozat \& D. R. Montello (Eds.), Spatial Cognition V: Reasoning, Action, Interaction (Vol. 4387, pp. 229-248): Springer.

Winkler, C., Broscheit, M., \& Rukzio, E. (2011). NaviBeam: Indoor Assistance and Navigation for Shop-ping Malls through Projector Phones. Proceedings of the CHI 2011 Workshop on Mobile and Personal Projection.

Wolbers, T., \& Hegarty, M. (2010). What determines our navigational abilities? Trends in cognitive sciences, 14(3), 138-146.

Wood, J. N. (2011). A Core Knowledge Architecture of Visual Working Memory. Journal of Experimental Psychology-Human Perception and Performance, 37(2), 357-381.

Xu, Q., Li, L., Lim, J. H., Tan, C. Y. C., Mukawa, M., \& Wang, G. (2014). A wearable virtual guide for context-aware cognitive indoor navigation. Proceedings of the $16^{\text {th }}$ International Conference on Human-Computer Interaction with Mobile Devices \& Services.

Yonelinas, A. P. (2002). The nature of recollection and familiarity: A review of 30 years of research. Journal of Memory and Language, 46(3), 441-517. 
Zelek, J. S. (2005). Seeing by touch (haptics) for wayfinding. International Congress Series, 1282, 1108-1112. doi: http://dx.doi.org/10.1016/j.ics.2005.06.002 